Check for updates

Cite this: RSC Adv., 2017, 7, 47464

Received 2nd August 2017

Accepted 3rd October 2017

DOI: $10.1039 / c 7 r a 08515 j$

rsc.li/rsc-advances

\section{Recent advances in hybrid measurement methods based on atomic force microscopy and surface sensitive measurement techniques}

\begin{abstract}
Stephan Handschuh-Wang, ${ }^{a}$ Tao Wang ${ }^{b}$ and Xuechang Zhou iD *a
AFM was proposed in the 1990 s as a tool to determine the topography at a sub-micrometer level. Since then AFM proved to be a valuable tool in material science and physical chemistry. Soon after successful application of AFM, it was employed for biological applications. The success of AFM can be ascribed to the high sensitivity, versatility, and resolution (1000 times higher resolution compared to optical microscopy). However, it intrinsically lacks amongst others chemical sensitivity. These limitations of AFM can be overcome by coupling it to orthogonal (complementary) optical (i.e. microscopy techniques) or non-optical techniques (i.e. Kelvin method). Such combinations are capable to garner information on morphological and mechanical properties of a sample together with fluorescence, spectroscopic (IR/Raman/fluorescence), electric, temperature or conductivity. Recently, advances in microscopy and super-resolution techniques enable the collection of even more than 2 measurands. Furthermore, new combinations, such as the photothermal-induced resonance (PTIR), a combination of IR spectroscopy and AFM, and scanning near-field ellipsometry microscopy (SNEM), a combination of ellipsometry and AFM, are actively researched, widening the adoptability of orthogonal combinations relying on AFM, while researchers devote tremendous effort to enhance established hybrid methods, such as tipenhanced Raman spectroscopy (TERS). Here, we introduce hybrid measurement techniques, relying on AFM and complementary techniques. The main focus of these hybrid techniques is on the combination with optical techniques as there has been much progress in the past few years and we can contribute our expertise in this field. For each combination, the working principle is explained briefly and applications of such combinations are pointed out. Finally, a short comprehension is given with basic
\end{abstract}

${ }^{a}$ College of Chemistry and Environmental Engineering, Shenzhen University, Shenzhen 518060, P. R. China. E-mail: xczhou@szu.edu.cn; Fax: +86-75526536141; Tel: +86-755-26536627
${ }^{b}$ Functional Thin Films Research Center, Shenzhen Institutes of Advanced Technology, Chinese Academy of Sciences, Shenzhen 518055, P. R. China

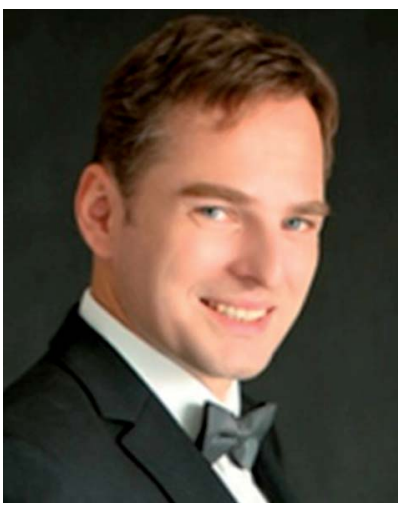

Stephan Handschuh-Wang obtained his Diploma degree in physical chemistry from the University of Technology Dortmund in 2010. He pursued his PhD with Professor Holger Schönherr at the University of Siegen on the study of soft matter interfaces and obtained his PhD in 2016. Dr HandschuhWang is currently a postdoctoral fellow of Prof. Xuechang Zhou's group at Shenzhen University. His previous and current research interests are focused on phenomena at the interface of preferably soft matter, in particular, the characterization and application of novel functional interfaces.

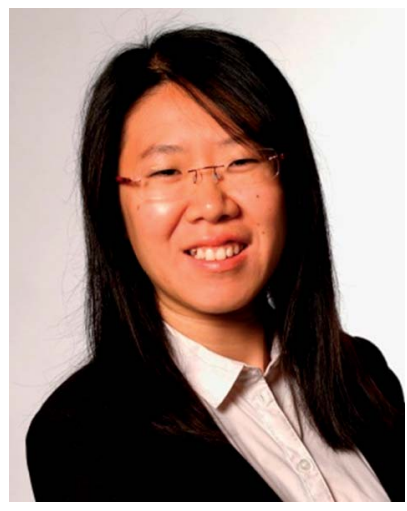

Tao Wang obtained her bachelor and Master degree in materials science and engineering from the Dalian University of Technology in 2011. She pursued her PhD with Professor Xin Jiang at the University of Siegen on the study of surface science and technology and obtained her PhD in 2015. Dr Tao Wang is currently a postdoctoral fellow of Prof. Yongbing Tang's group at Shenzhen Institutes of Advanced Technology, Chinese Academy of Sciences. Her previous and current research interests are focused on surface coating, surface functionalization and interactions of molecules in applications of mechanical engineering, materials science, and biomedicine. 
information on resolution and field of application of all major combinations. We envision that this review is helpful to those confronted with combined measurements for the first time, for experienced researchers, needing quick access to recent literature as it may convey new ideas for problems in analysis, as well as for researchers, who develop novel combined methods.

\section{Introduction}

Advances in science are closely related to advances in the design and construction of analysis tools. Here, confocal laser scanning microscopy should be mentioned with its unprecedentedly high lateral resolution and optical slicing ability. A second measurement technique, which made further advances possible, was atomic force microscopy. The atomic force microscope (AFM) rapidly emerged as an invaluable tool for direct measurement of topography and intermolecular forces with atomic resolution for a broad spectrum of applications, such as electronics, ${ }^{\mathbf{1 - 3}}$ semiconductors, ${ }^{\mathbf{4}}$ materials and manufacturing, ${ }^{5}$ polymers, ${ }^{6}$ biology, and biomaterials. ${ }^{7}$ An AFM is able to measure a sample at ambient conditions ${ }^{8}$ and even in liquid $^{\mathbf{9 - 1 1}}$ or buffer solutions, ${ }^{\mathbf{1 2}}$ which is in contrast to scanning tunneling microscopy (STM), scanning electron microscopy (SEM) and transmission electron microscopy (TEM). This feature renders AFM especially useful for in vitro measurements of biological samples. ${ }^{\mathbf{1 3}}$ Furthermore, AFM can employ several operation modes, which yields additional capabilities and makes it a powerful tool for several applications. These operation modes are for example contact mode, intermittent mode (topography), lateral force microscopy (friction) and noncontact mode, force modulation (elasticity) and phase imaging (dissipation). Nanoindentation can be utilized to determine in situ the mechanical properties of specific points and areas of a sample without change of the cantilever, tip, measurement equipment, and sample. ${ }^{\mathbf{1 4 - 1 6}} \mathrm{A}$ widespread technique is force

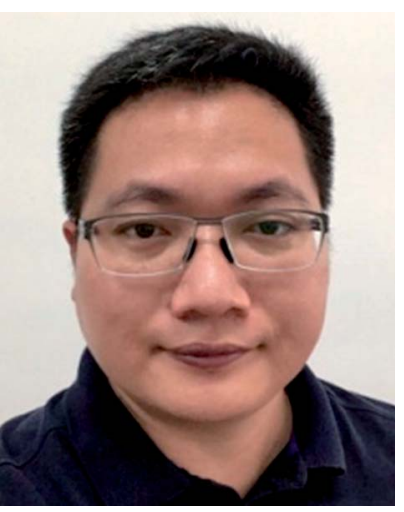

Prof. Xuechang Zhou is currently an Associate Professor of College of Chemistry and Environmental Engineering at Shenzhen University. Prof. Zhou received his B.S in polymer chemistry (Advisor: Prof. Guangzhao Zhang) at the University of Science and Technology of China in 2005, and Mphil and PhD degrees in Department of Chemistry (Prof. Bo Zheng's group) at the Chinese University of Hong Kong in 2007 and 2010, respectively. From 2010 to 2014, he worked in Prof. Zijian Zheng's group as a Postdoctoral at the Hong Kong Polytechnic University focusing on the development of flexible and wearable electronics, and surface patterning technique. He joined the College of Chemistry and Environmental Engineering at the Shenzhen University as an Associate Professor in 2014. His research interests included flexible and stretchable electronics, sensors, surface analysis and environmental engineering. modulation microscopy (FMM), which is utilized to characterize mechanical properties of polymers or composite material to detect inhomogeneities or contaminations. ${ }^{17,18}$ In biological regime, AFM can be utilized to determine biomechanical properties, changes in mechanical properties of cells, membranes and other biomaterials. ${ }^{19,20}$ AFM can be utilized to determine the adhesion of the tip and the surface. By functionalizing the tip/tip-apex with different probes, such as nanoparticles ${ }^{21}$ or molecules, ${ }^{22,23}$ interaction forces between two surfaces, a surface and a biological molecule or two biological molecules (single molecule force spectroscopy (SMFS)) can be determined. ${ }^{24,25}$ AFM is utilized in a wide range of disciplines in natural sciences, including solid state physics (e.g. identification of atoms at surfaces, sensor of electric charge and dynamic lateral friction microscopy), ${ }^{26}$ semiconductor science (e.g. topography, conductivity and current sensing), ${ }^{27}$ molecular engineering (e.g. nano-manipulation), ${ }^{28}$ polymer chemistry (e.g. surface structure, chemical sensing and viscoelasticity $)^{\mathbf{2 9 , 3 0}}$ surface chemistry (e.g. roughness, height, friction and surface rheology), ${ }^{31}$ molecular biology (e.g. DNA and chromosome studies and monitoring biological processes), ${ }^{32,33}$ cell biology (cell imaging \& mechanical properties) ${ }^{34}$ and medicine (e.g. virology and tissue/organ imaging). ${ }^{35,36}$ Besides, new operation modes for the AFM are actively developed, such as scanning probe lithography ${ }^{37-40}$ and non-contact mode, and nowadays multiparametric AFM imaging is possible, ${ }^{\mathbf{4 1}}$ further diversifying the field of application.

However, AFM as a stand-alone technique retains a few limitations. Identification of the surface termination or chemical composition of a sample is beyond its reach, as AFM is an indirect measurement technique it intrinsically lacks chemical information. ${ }^{42}$ Furthermore, AFM resembles a technique relying on the analysis of a surface by a probing stick (cantilever), without seeing the morphology directly. This often results in challenging and time-consuming search for a specific region of interest (ROI), similar to finding the light-switch in a dark room. Hence, many novel AFMs are equipped with a camera, facilitating laser alignment and ROI identification. Yet, the camera is typically only capable of ascertaining a rough knowledge of the surface (mm range), whereas the AFM measures in $\mu \mathrm{m}$ and $\mathrm{nm}$ range. Therefore, combining AFM with optical microscopy, confocal laser scanning microscopy or super-resolution techniques provide an advantage over stand-alone AFM techniques.

A combination of AFM and an orthogonal optical measurement technique, such as confocal laser scanning microscopy (CLSM), ${ }^{\mathbf{4 3}, 44}$ Raman (tip-enhanced Raman spectroscopy $(\text { TERS) })^{45}$ or infrared (photothermal-induced resonance (PTIR) $),{ }^{46}$ or non-optical techniques, like scanning thermal force microscopy, ${ }^{47}$ makes it possible to detect chemical information, ${ }^{48}$ identify the chemical composition ${ }^{49}$ or detect the thermal conductivity $^{50}$ at high (lateral) resolution, which also offers new 
opportunities to gather multidimensional data from a given system..$^{51,52}$ In combinations of an optical technique with AFM, both techniques retain their individual capabilities, which means the resolution and the information garnered by a method stays the same (obviously, the case for CLSM) or is in some cases improved (for tip-enhanced methods, such as TERS and PTIR). Furthermore, new capabilities arising from the versatility of the AFM can expand the capability of a combined setup. For example, an AFM-CLSM combination can make use of fluorescence modulation effects, which arise from the AFM tip in the vicinity of fluorescent molecules. These effects can either be enhancement ${ }^{53,54}$ or quenching ${ }^{55,56}$ of fluorescence, leading to a change in the excited state lifetime ${ }^{57}$ and can be utilized to enhance the spatial resolution of the CLSM/FLIM (fluorescence lifetime imaging microscopy). ${ }^{58}$

Tremendous effort is concentrated on the implementation of non-optical methods, such as Kelvin probe ${ }^{59}$ or thermal microscopy ${ }^{50}$ into the AFM. These combined methods allow concomitant measurement of topography with a localized surface potential, ${ }^{60}$ thermal information ${ }^{61}$ or thermal conductivity ${ }^{50}$ which is important for material science and engineering, for example, mapping of semiconductors and their work functions. ${ }^{62}$

This review covers the combination of optical and nonoptical surface sensitive techniques with the AFM. An overview of the hybrid methods discussed in this article is given in Fig. 1. For comparison, firstly, super-resolution techniques are introduced, which are capable of increasing the resolution of optical images, with its advantages and drawbacks. Subsequently, the main focus is on combinations of optical methods, especially fluorescence microscopy, and CLSM, as with these examples the alignment, an important issue for hybrid methods, can be introduced easily. Afterwards, combinatory methods based on AFM-CLSM are introduced, such as the combination of AFM with FLIM and stimulated emission depletion microscopy (STED), which are viable and recently developed candidates for predominantly addressing biological samples. Important representatives beside fluorescence-based methods are introduced hereafter, for example combination of AFM with Raman, tip-enhanced Raman scattering (TERS), and infrared spectroscopy, photothermal-induced resonance (PTIR). Thereafter, combinations of AFM with non-optical methods are introduced, such as the combination of AFM with Kelvin's method (a method for determining the contact potential difference), Kelvin probe force microscopy (KPFM), and with thermal conductivity measurement, scanning thermal microscopy (SThM). Examples out of scientific publications illustrate the applicability of these orthogonal measurement techniques and their scope. Finally, the advantages and disadvantages of these techniques are evaluated and the recent progress of hybrid methods relying on AFM is assessed.

As this review article focuses on the recent advances of hybrid methods and novel hybrid methods since 2009, this article cannot cover the basic principle of the AFM and its different measurement modes. The interested reader is referred to the well-established literature regarding AFM, for example the books by Eaton, West: Atomic force microscopy ${ }^{63}$ for a more theoretic overview and Schönherr, Vancso: Scanning Force Microscopy of Polymers ${ }^{64}$ for a more practical introduction and the recent inspiring reviews by Zhong and Yan $^{65}$ (AFM for nanomaterial research), Allison et $a l^{66}$ (AFM for biological samples) and Dufrêne et al. ${ }^{67}$ (imaging modes, AFM for molecular and cell biology).

\section{Combination of AFM with optical surface sensitive methods}

Well-established methods in biology and nanotechnology, i.e. for cell-imaging or imaging of nanoparticles, are fluorescence microscopy and confocal fluorescence microscopy or confocal laser scanning microscopy (CLSM) ${ }^{68,69}$ CLSM was first patented in 1957 by Marvin Minsky. ${ }^{70}$ Its impact for research applications arises from the drastic improvement of optical resolutions to an unprecedented level of up to $180 \mathrm{~nm}$ lateral $(x-, y)$ and up to

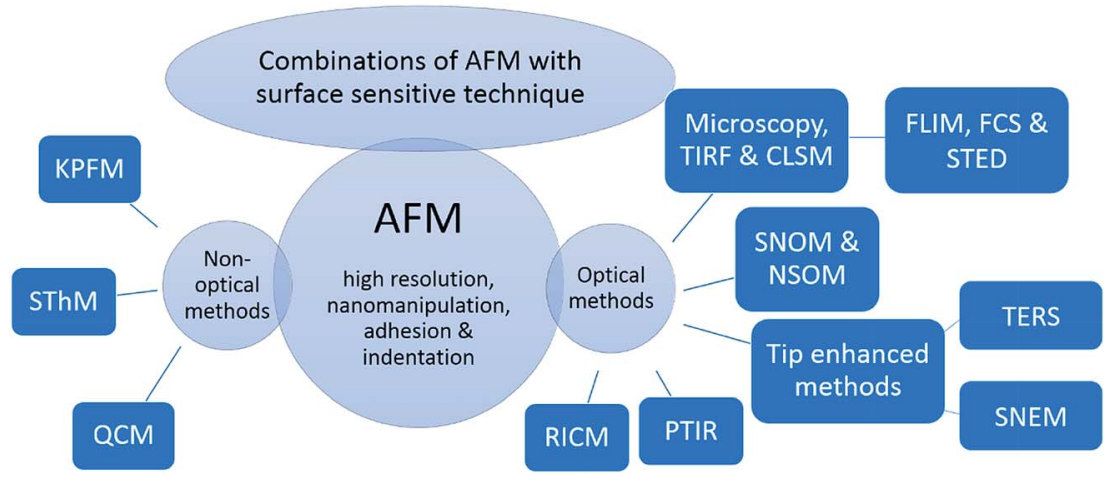

Fig. 1 Overview of hybrid measurement techniques based on a surface sensitive technique and AFM. The abbreviations in this figure denote for the following: KPFM - Kelvin probe force microscopy, SThM - scanning thermal microscopy, QCM - quartz crystal microbalance, TIRF - total internal reflection fluorescence, CLSM - confocal laser scanning microscopy, FLIM - fluorescence lifetime imaging microscopy, FCS - fluorescence correlation spectroscopy, STED - stimulated emission depletion, SNOM - scanning near-field optical microscopy, NSOM - near-field scanning optical microscopy, TERS - tip-enhanced Raman scattering, SNEM - scanning near-field ellipsometry microscopy, PTIR - photothermal-induced resonance and RICM - reflection interference contrast microscopy. 
$500 \mathrm{~nm}$ axial $(z)$ resolution due to the spatial filtering technique. ${ }^{71,72}$ This technique allows optical slicing of the sample and construction of a three-dimensional image without collection of out of focus light. ${ }^{73}$ This feature allows to image for example cells, tissues, 3D scaffolds/sponges, which showed promise in copious application, including microfluidics and flexible electronics, ${ }^{74-77}$ or nanostructures and subsequently illustrate them 3-dimensional. ${ }^{78-80}$ Nowadays, the research is shifting towards nanotechnology (for example nanocontainers) and fundamental biology of cells, which are in a size-range of tens to few hundred nm. ${ }^{81}$ Here, optical microscopy methods lack sufficient resolution due to the diffraction limit described by Abbe. ${ }^{82}$ Several attempts to overcome the diffraction limit were successful. These methods are introduced briefly in the following paragraph.

\subsection{Super-resolution techniques}

The general name of approaches overcoming the resolution limit is super-resolution fluorescence microscopy. As an emerging method, the super-resolution reversible saturable optical linear (fluorescence) transitions (RESOLFT) microscopy has to be mentioned, ${ }^{83,84}$ as it is suitable for in vivo studies. ${ }^{85}$ STED (stimulated emission depletion) and ground state depletion (GSD) microscopy rely on the working principle of RESOLFT. ${ }^{86}$ As STED is a well-established method, since Stefan Hell received the Nobel Prize in Chemistry in 2014 for his outstanding work in superresolved microscopy, ${ }^{\mathbf{8 7}}$ this technique is shortly introduced here. However, other - not less intriguing -super-resolution techniques include PALM ${ }^{88}$ (Photoactivated Location Microscopy), FIONA ${ }^{89}$ (Fluorescence Imaging with One Nanometer Accuracy), a method for accurately determining the position of a fluorophore by ascertaining the center of its emission pattern, ${ }^{89}$ and STORM $^{\mathbf{9 0}}$ (Stochastic Optical Reconstruction Microscopy), a stochastic approach based on FIONA and relying on the transfer of the majority of fluorophores to a non-fluorescent dark state (see also the topical review of Hell et $a{ }^{85}$ ). In STED, two wellaligned lasers are used. The first laser possesses the typical Gaussian beam profile and is used to excite the fluorophores in the sample, whereas the second laser possesses a donut-shaped beam profile and is used to de-excite all but the fluorophores in the center of the beam. Afterwards, the fluorescence emission of molecules in this small region (smaller than diffraction limit) is detected. As STED is a scanning technique, the image is generated by moving the beams over the sample while recording the fluorescence intensity. A lateral resolution of down to $2.4 \mathrm{~nm}$ was reported recently. ${ }^{91}$ Typically, the resolution is in the order of tens of nanometers. ${ }^{92}$ The resolution can be adjusted by the intensity of the depletion pulse, the lateral STED-enhanced resolution/PSF (point spread function) is given by eqn (1). ${ }^{93}$ The axial resolution, however, remains for a typical experiment rather low (approx. 40-500 nm). ${ }^{94}$

$$
d_{\mathrm{STED}}=\frac{\lambda}{2 \mathrm{NA} \times \sqrt{1+I / I_{\mathrm{Sat}}}}
$$

Here, $\lambda$ is the wavelength of the excitation light, NA is the numerical aperture of the objective, $I$ is the intensity of depletion light, and $I_{\text {sat }}$ is a fluorophore-dependent parameter defined by the intensity of light required to suppress $50 \%$ of fluorescence. During STED measurements it is feasible to determine the excited state lifetime of a fluorophore or even multiple fluorophores, ${ }^{95}$ but the initial part of the fluorescence decay is difficult to analyze as it is compromised by the action of the STED beam, rendering the decay multi-exponential. It contains fluorescence photons from fluorophores located further away from the doughnut zero and is therefore not superresolution. ${ }^{96}$ Hence, the first two nanoseconds of the timecorrelated single photon counting (TCSPC) decay are typically discarded under the premise that organic dye molecules have a fluorescence lifetime in the order of 2 to $5 \mathrm{~ns} .{ }^{95}$ The feasibility of STED/FLIM was shown by Bücker et al. They used a twochannel STED imaging system and fluorescence lifetime separation was applied. Tubulin and lamin were immunostained with ATTO $647 \mathrm{~N}$ and KK 114, respectively. By fitting each pixel with a biexponential decay of fixed lifetimes (1.8 ns and 3.1 ns) the structures of tubulin and lamin were well separated..$^{95}$

The drawbacks of STED are, that (a) only a small variety of fluorophores may be used for STED due to their stability against photo-bleaching ${ }^{97}$ and (b) short fluorescence lifetimes i.e. of samples with a high concentration of reporter dye (selfquenching) cannot be determined with a high accuracy due to the STED-induced decay. ${ }^{98}$ Also the analysis of the amplitudes will yield in falsified results as the decay is not used between 0 and 2 ns after the excitation pulse. Here, the normal fluorescence lifetime imaging microscopy has an advantage over the STED/FLIM as the instrument response function (IRF) is well defined and for fitting the excited state lifetimes the whole decay may be used, which yields in high accuracy of short lifetimes and a quantitative analysis of different populations. Therefore, other techniques and combinations of measurement techniques are developed or are used.

\subsection{Coupling far-field optical microscopy and its variations with the AFM}

2.2.1 How to combine optical microscopy and AFM? The sample preparation for fluorescence microscopy and confocal laser scanning microscopy is facile and fast. ${ }^{99}$ However, often staining or usage of reporter dyes is needed (see for example the inspiring topical review by Dean and Palmer ${ }^{\mathbf{1 0 0}}$ ). Samples can be measured in solution, which makes it a versatile method for analyzing biological samples at physiological conditions. ${ }^{\mathbf{1 0 1}}$ Therefore, CLSM and fluorescence microscopy are frequently utilized in biology ${ }^{\mathbf{1 0 2 - 1 0 4}}$ (e.g. cellular structure, localization of ions/macromolecules and tracing specific cell) and medicine ${ }^{\mathbf{1 0 5 , 1 0 6}}$ (e.g. dentistry, stem cells, human cornea and in vivo confocal microscopy). The applications are not confined to biological samples, rather CLSM is utilized in a broad range of applications, including polymer chemistry ${ }^{\mathbf{1 0 7}}$ (diffusion coefficient) and surface chemistry ${ }^{\mathbf{1 0 8}}$ (adsorption of (macro)molecules).

Fig. 2 shows different acquisition modes. The two major methods of data acquisition of a combined measurement are either concomitant (Fig. 2a) or successive (Fig. 2b). ${ }^{58,109}$ Some 
(a)
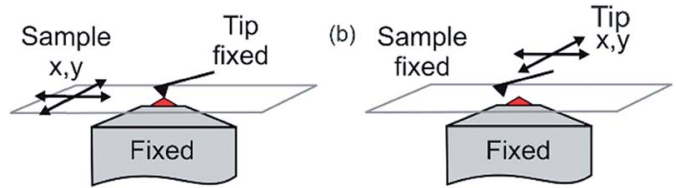

(c)
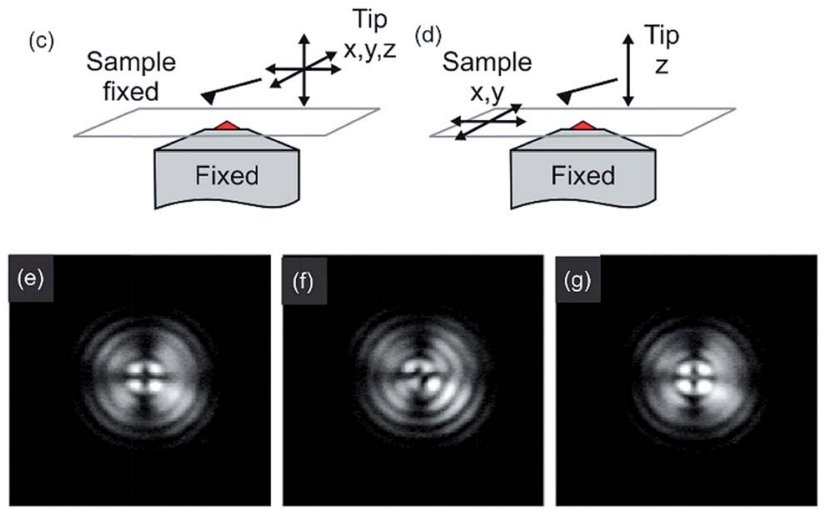

Fig. 2 Modes of operation of the atomic force fluorescence microscope (AFFM): (a) simultaneous imaging by scanning the sample between a fixed tip and a fixed focus; (b) AFM imaging by lateral tip scanning with a specific $\mathrm{ROI}$ in the excitation volume; (c) forceextension imaging with the tip moving in three dimensions; (d) forceextension imaging with the tip ramping perpendicular to the surface and the sample moving in lateral directions. Reprinted with permission from ref. 44 Copyright (2005) John Wiley and Sons. (e-f) Beam diagnostic camera images of back-reflected light from the sample surface with the AFM tip retracted (e), with AFM tip visible as a distortion (f) and after alignment (g). For all images, the focus had been moved towards the AFM tip to show the distortion of the pattern more clearly. Reprinted with permission from the authors and ref. 110 Copyright (2010) Society of Photo-Optical Instrumentation Engineers.

measurements or analysis are even made comparative, meaning AFM and optical measurements may not be at the same position of the sample (not aligned). A researcher should decide carefully if it is favorable to measure simultaneously or execute the measurements successively. A combined measurement may save time and an interaction of the tip with the sample may be observed due to tip-enhanced fluorescence or tip-induced quenching. ${ }^{57,110}$ This may be desired to determine the location of a dye molecule on a surface or in a polymer matrix. On the other hand, the determination of fluorescence properties of fluorophores and the topography measurement have to be conducted successively to extirpate undesired changes in the fluorescence properties of the fluorophore.

To combine far-field optical microscopy techniques, such as fluorescence microscopy, confocal microscopy and FLIM or other methods, such as total internal reflection fluorescence (TIRF), fluorescence correlation spectroscopy (FCS) and superresolution techniques, with the AFM a specific setup is needed, which has to be aligned perfectly (up to a few nanometers). The combined AFM-optical microscopy technique consists out of a sample scanning AFM and an inverted microscope (e.g. CLSM). The microscope can be equipped with different excitation wavelengths. For optical detection with high resolution, an (oil) immersion objective with a high numeric aperture ( 1.45 or even higher) can be utilized. The link between the two measurement systems is the AFM sample stage, which is mounted directly on the inverted microscope chassis replacing the original sample stage. The sample stage has to be mounted carefully onto the inverted microscope, especially, taking into account the necessary space needed for the objective of the microscope, as well as its operating distance. It might be necessary to insert spacers. This setup allows measuring concomitant or successively optical microscopy and AFM.

For alignment of the orthogonal methods, first, the cantilever tip is adjusted to the cross hairs in the eyepiece of the microscope by manual positioning of the sample stage. Now, the cantilever tip is brought into contact with the surface and the microscope is adjusted to measure backscattering light. Keep in mind to reduce the excitation intensity to protect the detector. Acquired images of the backscattering of the cantilever indicate the position of the tip. The tip apex is typically visible in the backscattering images as a bright spot. ${ }^{58}$ Alternatively, the scattering of the incident light, which can be observed by a CCD camera (beam diagnostic camera, see Fig. 2e-g), can be utilized for fine adjustment, if the tip apex is not visible in the backscattering image. ${ }^{109}$ The quality of alignment is in this process clearly visible in the distortion of the reflected light originating from the retracted tip (Fig. 2f). Rather a strong distortion of the symmetry is observed for a misaligned setup, while high symmetry of the backscattered light is obtained for a wellaligned (AFM tip in the center of distortion pattern) setup, as shown in Fig. $2 \mathrm{~g}$.

2.2.2 AFM-combined with fluorescence microscopy and confocal microscopy. Numerous attempts to combine the AFM with optical fluorescence microscopy have been made. An early combination of confocal microscopy with an AFM was constructed in 1994 by Neagu et al. ${ }^{111}$ They were able to measure human lymphocytes, which were sequentially labeled with FITC and immunogold, with a combination of AFM and optical fluorescence microscopy. The images showed good correlation and they were able to determine optimal imaging conditions for both the AFM and the microscopy by varying the thickness of immunogold and silver enhancement. ${ }^{\mathbf{1 1 1}}$ Another combination of optical microscopy with AFM was developed by Lieberman et $a l .{ }^{112}$ They integrated near-field optical microscopy and farfield microscopy into an AFM. The instrument had the capability to measure normal force and shear force, as well as subdiffraction limit fluorescence intensity (NSOM) and it was capable to optically slice the desired ROI (confocal microscope). ${ }^{112}$ Consequently, after these first attempts to combine the two methods, such hybrid instruments were utilized in biological imaging of cells, filaments, proteins, cytoskeleton and nucleotides, and in biophysics, for example for the analysis of structural and dynamic changes in lipid bilayers. ${ }^{113-121}$

A problem for combined AFM and microscopy measurements in the past was the presence of background noise in microscopy images, which is related to the laser utilized for the AFM optical lever. ${ }^{\mathbf{1 2 2}}$ This is bypassed either by measuring consecutively or by measuring concomitant while using a highquality filter. ${ }^{\mathbf{1 2 2}}$ While utilizing filters, a potentially useful part of the spectrum cannot be analyzed due to the blocking of these wavelengths. This is especially a problem for microscopic 
systems, utilizing the emitted fluorescence light for generation of spectra. By employing a laser with a wavelength of approx. $1050 \mathrm{~nm}$ for the AFM, the background in microscopy images as well as problems with photobleaching or unspecific excitation of the sample can be avoided. ${ }^{123}$ Kassies et al. designed such a combination of an AFM with a confocal microscope (atomic force fluorescence microscope (AFFM)), where the AFM laser was shifted to a wavelength of $1050 \mathrm{~nm}$. They constructed an AFFM with the capability of measuring simultaneously topography and fluorescence images, and the capability to measure fluorescence spectra at specific points of interest. They showed that they can measure on membrane fragments from Rhodobacter sphaeroides, containing both LH1 and LH2 (LH = light-harvesting pigment-protein complex). ${ }^{123}$ Furthermore, they demonstrated that measurements in a combined optical and force extension/nanomanipulation mode is possible with their setup. ${ }^{123}$

As shown previously, the exact alignment may be difficult and time-consuming. Another problem during a combined experiment is the drift in an AFM during measurement, ${ }^{121}$ which necessitates keeping an eye on the alignment of the measurement equipment. Timmel et al. utilized an alignment correction method, which uses the cantilever tip as a reference landmark. ${ }^{121}$ At first, they validated their method with beads in a complex artificial sample and subsequently, the method was utilized to maintain alignment during measurement of membrane structures in fixed and living human fibroblasts (biological sample). They showed that the fluorescence signals and the topographic structures were well-aligned and that nanoscale membrane structures could be related to the fluorescence spot. ${ }^{121}$

Lately, Staunton et al. presented a method for quantitative mechanical characterization of soft, heterogeneous samples in 3D via a combination of AFM, CLSM and finite element analysis (FEA). They showed the capability of their combined technique for the quantification of the elastic properties of cells (here metastatic breast adenocarcinoma) in an extracellular matrix (here collagen hydrogels). With this method, it is possible to decouple the response of a cell from its soft surrounding (extracellular matrix, ECM). By this means, although the response of the indentation experiment is based on both, the cell and the soft surrounding, the elastic properties of the cell and the ECM can be determined quantitatively. ${ }^{124}$

2.2.3 A combination of AFM with fluorescence lifetime imaging microscopy (FLIM). Fluorescence light possesses both frequency and time-domain. A combination of the AFM with a confocal fluorescence microscope working in the time domain (i.e. time-correlated single photon counting) enables the direct measurement of topography, fluorescence intensity and fluorescence lifetime. Since 1926 it has been possible to determine the excited state lifetime of fluorescence $(\tau)$, another basic fluorescence parameter. ${ }^{125}$

The fluorescence lifetime (decay time, eqn (7)), $\tau_{\mathrm{F}}$, can be defined by use of a simple kinetic scheme (first-order rate constants), where all radiative (fluorescence, eqn (3)) and all nonradiative pathways (inter system crossing (ISC), internal conversion (IC), dissociation) have to be considered.

$$
\begin{gathered}
\mathrm{M}+h \nu \rightarrow{ }^{1} \mathrm{M}^{*} \times I_{\mathrm{a}} \text { (absorption) } \\
{ }^{1} \mathrm{M}^{*} \rightarrow \mathrm{M}+h \nu \times k_{\mathrm{R}} \text { (fluorescence) } \\
{ }^{1} \mathrm{M}^{*} \rightarrow{ }^{3} \mathrm{M}^{*} \times k_{\mathrm{ISC}} \text { (intersystem crossing) } \\
{ }^{1} \mathrm{M}^{*} \rightarrow \mathrm{M} \times k_{\mathrm{IC}} \text { (internal conversion) } \\
{ }^{1} \mathrm{M}^{*} \rightarrow \text { products } \times k_{\mathrm{D}} \text { (dissociation, bleaching) }
\end{gathered}
$$

Here, $k_{\mathrm{i}}$ depicts the rate constants of the processes shown in eqn (3)-(6). From the steady-state analysis of the eqn (3)-(6) the fluorescence decay time $\tau_{\mathrm{F}}$ is given by eqn (7).

$$
\tau_{\mathrm{F}}=\left(k_{\mathrm{R}}+k_{\mathrm{IC}}+k_{\mathrm{ISC}}+k_{\mathrm{D}}\right)^{-1}
$$

There are several techniques to determine the decay times of fluorescence. The most common technique is TCSPC. It relies on the accurate time measurement of single photon events (e.g. fluorescence). The laser pulse serves as a reference for the start time, whereas the stop time is determined by the fluorescence photon. These photon detection events are binned and converted into a histogram. For this histogram, a small time bin down to $1 \mathrm{ps}$ is used. The photon events are collected by adding +1 in the time bin proportional to the start-stop time. After many cycles, the distribution of such detection times builds up. ${ }^{126-128}$ Afterwards, the data can be analyzed by eqn (8) (exponential decay).

$$
A(t)=\sum_{i} \alpha_{i} \mathrm{e}^{-\frac{t}{\tau_{i}}}
$$

The fluorescence lifetime values can yield information about the molecular microenvironment of a fluorescent molecule, i.e. viscosity, ${ }^{129,130} \mathrm{pH},{ }^{131}$ temperature, ${ }^{132}$ polarity ${ }^{133,134}$ and solvation. ${ }^{135}$ Furthermore, the excited state lifetime of a fluorophore can be altered by ionic strength, ${ }^{136}$ oxygen concentration, ${ }^{137}$ binding to macromolecules, changes in protein orientation, change in protein folding, ${ }^{138}$ a critical binding reaction ${ }^{139}$ and the proximity of molecules that can deplete the excited state by resonance energy transfer (RET).$^{138,140}$ Therefore, fluorescence lifetimes can be utilized as indicators of these parameters. There are various applications utilizing fluorescence lifetime, i.e. in calcium and other chemical detection, ${ }^{141}$ clinical detection $^{142}$ and determination of transport processes (diffusion). ${ }^{143,144}$ Although FLIM is typically featured in biological studies, it also finds application in polymer science and surface analysis. ${ }^{109,145}$ A combination of FLIM with AFM potentially yields information of biological processes occurring inside cells, which would not be possible without FLIM.

Recently, a few articles concerning themselves with this kind of combination, which belong to an a-SNOM approach, were published. An advantage, which was exploited in these articles, was the higher optical resolution due to short ranged tipinduced quenching and inherent fluorescence lifetime decline. Schulz et al. dried a diluted sample of ATTO $647 \mathrm{~N}$ on a clean glass slide. The density of the dye molecules was in the order of 1 molecule per $\mu \mathrm{m}^{2}$. During the measurement in the 
combined mode, the diffraction limited spots of the fluorophores show typical single molecule features like blinking and beaching. All fluorescence spots possessed a dark area in their center, which were typically in the order of 20-40 nm FWHM. The dark areas were attributed to energy transfer to the AFM tip. ${ }^{\mathbf{1 1 0}}$ Here, the excited state lifetime changed by advancing the tip towards the organic dye. A drop of excited state lifetime with decreasing distance was observed. Thus, this setup possesses the ability to measure with high resolution without the need of tedious and sophisticated super-resolution equipment, such as specialized dyes, optics, and lasers. A few years later, single digit nanometer resolution via the same approach was shown (see Fig. 3). Concomitantly, the fluorescence lifetime information yielded knowledge about the orientation of the dye molecules. Schulz et al. found that the molecular transition dipole moments lie parallel to the sample surface. ${ }^{58}$ AFM (intermittent mode) and FLIM were also used by Yoskovitz et al. ${ }^{57} \mathrm{CdSe}$ quantum dots coated with multiple shells (7.3 nm diameter) were placed onto a cleaned glass surface. AFM was measured in tapping mode isochronal with fluorescence intensity and the time-correlated single photon counting data. The obtained data showed a fluorescence enhancement for silicon tips at approximately $100 \mathrm{~nm}$ laterally away from the fluorophore. This data is in agreement with the literature, where the fluorescence enhancement is assigned to a field enhancement at the apex of the tip. ${ }^{\mathbf{1 4 6 , 1 4 7}}$ Furthermore they showed that excited state lifetime yields in an enhancement of the signal-tonoise ratio, and also significantly improved resolution. ${ }^{57}$

Lately, a combination of AFM-FLIM in a far field approach was utilized to study surface nanobubbles. ${ }^{109}$ Surface nanobubbles are nanocavities filled with gas (air), which nucleate at the interface between solids and liquids (typically water). For this approach water was stained with Rhodamine 6G (Rh6G). Rh6G appeared to be enriched at the liquid-gas interface, which developed during nanobubbles nucleation, as shown in Fig. 4b. The surface nanobubbles appeared brighter in the confocal fluorescence microscopy images than the interphase (glasswater) without nanobubbles (compare Fig. 4d and g). The fluorescence intensity image (bright nanobubbles) and the topography image of the nanobubbles were in good agreement. Here, the excited state lifetime analysis served as proof of a water-air interface. TCSPC decays measured at the location of
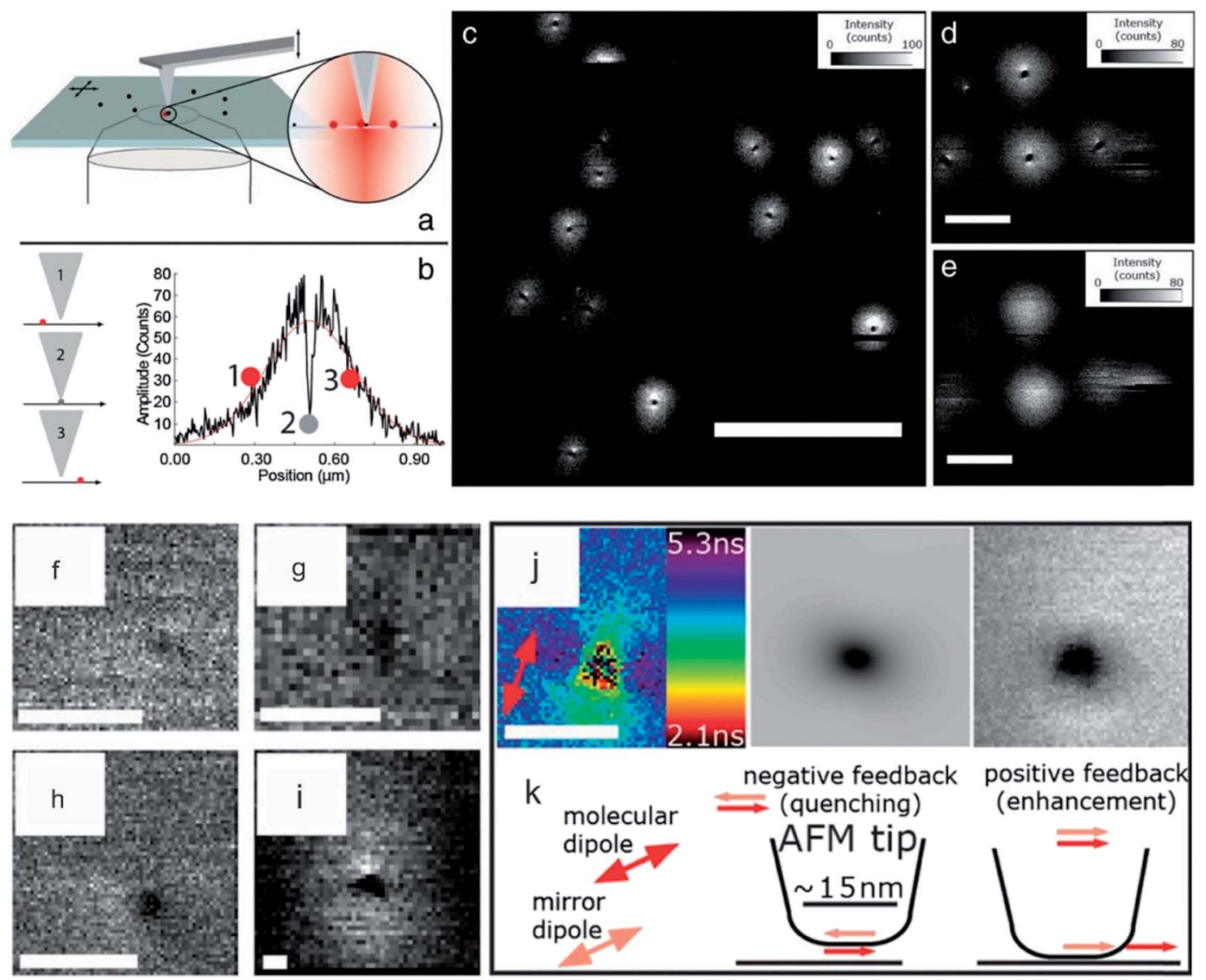

Fig. 3 (a) Scheme of hybrid AFM-FLIM setup. (b) Scheme of tip-induced quenching and course of intensity versus separation distance between tip and fluorophore. (c) Fluorescence intensity data of a sample containing single dye molecules (ATTO 655) with the AFM tip approached to the surface. (d) Magnification of (c). (e) Same ROI as in (d) is imaged with retracted tip. The scale bar for (c) is $2 \mu \mathrm{m}$ and for (d) and (e) $500 \mathrm{~nm}$. For (f), (g), (h) the FWHMs are $6.8 \mathrm{~nm}, 7.5 \mathrm{~nm}$, and $5.9 \mathrm{~nm}$, respectively. (i) Triangle shape due to a blunt tip. (j) Fluorescence lifetime distribution, the calculated fluorescence intensity and the measured fluorescence intensity of a quenching spot with a FWHM of $16 \mathrm{~nm}$ is shown. (k) Scheme of the physical mechanism leading to quenching. Reprinted from open-access article ref. 58 under the terms of the Creative Commons Attribution License. 
(a)

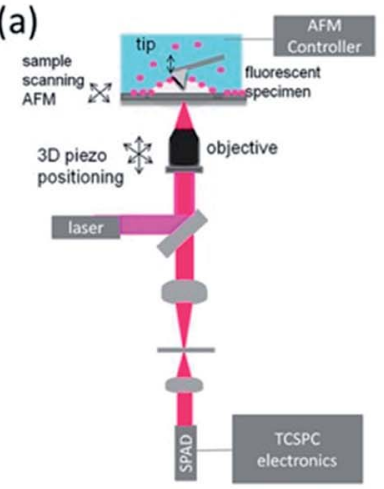

(b)
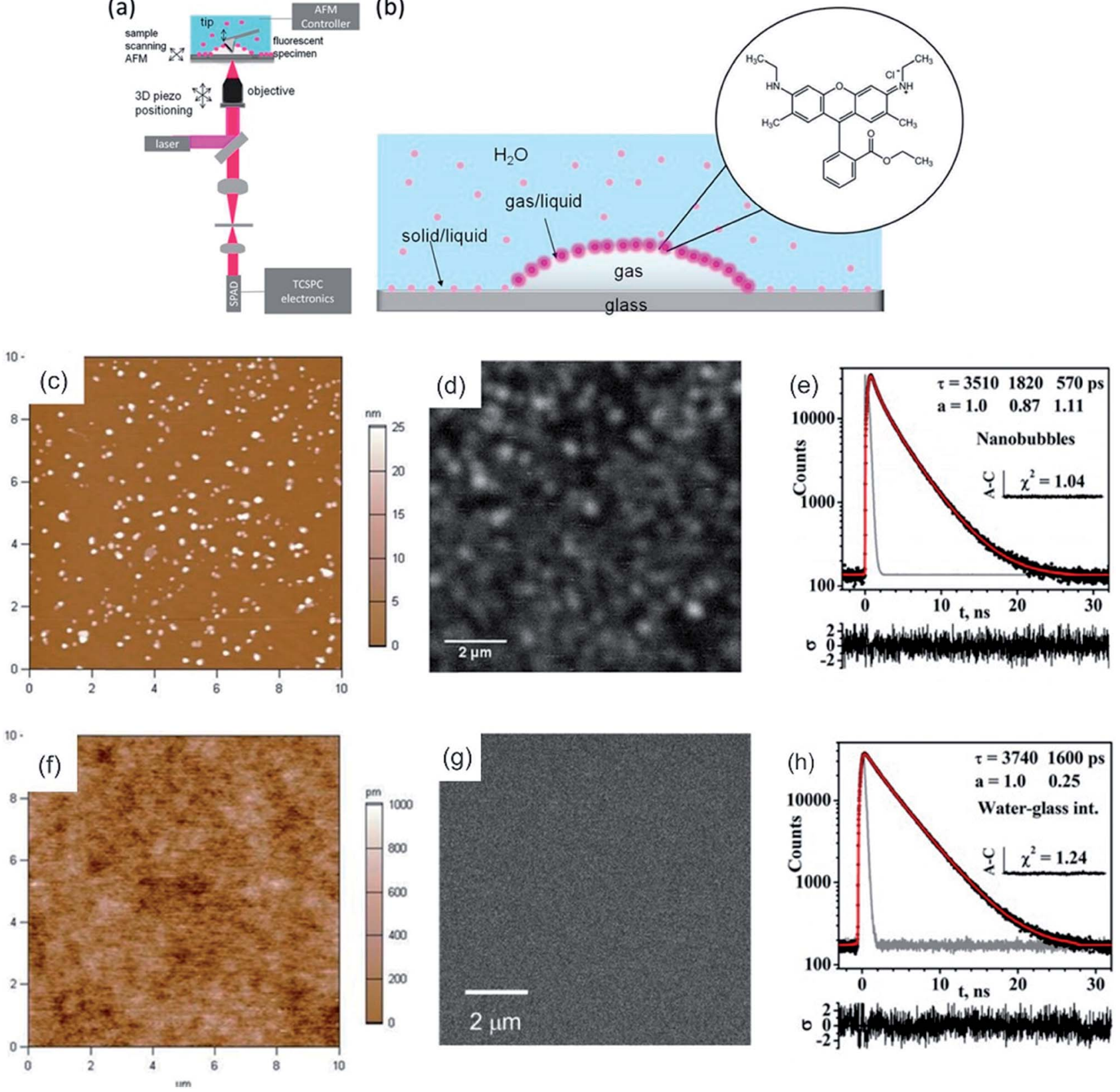

Fig. 4 (a) Scheme of the hybrid AFM-FLIM microscope. (b) Scheme of the partitioning of the fluorophore Rh6Gat the interface and in water. Nanobubbles nucleated by an ethanol-water exchange on the piranha-cleaned glass in an aqueous 850 nMRh6G solution: (c) AFM height image; (d) confocal fluorescence microscopy intensity image in the same ROI as in (c). (e) TCSPC fluorescence decay curve of Rh6G obtained from TTTR data of panel (d). (f) AFM height image and (g) confocal fluorescence microscopy intensity images of UV-Ozone cleaned glass after the ethanol-water exchange in aqueous Rh6G solution (850 nM). (h) TCSPC decay curve of Rh6G obtained from TTTR data in panel (g). Reprinted with permission from ${ }^{109}$ Copyright (2016) American Chemical Society.

a nanobubble yielded a tri-exponential decay, whereas TCSPC decays on the substrate-solid interface (without nanobubbles) yielded a bi-exponential decay (compare Fig. 4e and h). The third excited state lifetime, which was approx. $0.6 \mathrm{~ns}$, was attributed to the liquid-air interface, as this lifetime is not present in a sample without nanobubbles. ${ }^{109}$ Prior to this, the existence of this air/water interface was discussed in the academic community as these nanobubbles would be unstable due to high inner pressure (curvature). ${ }^{148}$
He et al. employed AFM correlated with single-molecule fluorescence intensity/lifetime imaging microscopy (their acronym: AFM-SMFLIM) to investigate interfacial electrontransfer processes of a mixed ( $\left(m-\mathrm{ZnTCPP} / \mathrm{TiO}_{2}\right.$ nanoparticles) $+m$-ZnTCPP)/cover glass sample. The correlated information of fluorescence blinking of each individual dye molecule and its fluorescence lifetime along with the surrounding topography explained the coupling strength inhomogeneity of each dye molecule with $\mathrm{TiO}_{2}$ nanoparticles (NPs) and its effect on 
electron transfer intermittency and thus, yielded molecular level understanding of the interfacial electron transfer reactivity. ${ }^{149}$

2.2.4 AFM coupled to fluorescence correlation spectroscopy (FCS). Only a few studies were published for combinations of AFM with FCS, ${ }^{150-154}$ although combinations of AFM and confocal fluorescence microscopy are utilized in the past 20 years (see above). This may be related to the application of FCS, which is utilized to measure quantitatively dynamic parameters, such as chemical reaction rates, ${ }^{155}$ diffusion coefficients, ${ }^{156}$ hydrodynamic radii, ${ }^{157}$ (average) concentration ${ }^{158}$ and singlettriplet transitions. ${ }^{159}$ Furthermore, reasons for its scarceness are a mismatch of acquisition times and difficulties with the alignment on the soft interface, which exacerbates the issue of drift in AFM during a combined measurement. These problems may be the reason for predominantly comparative measurements of AFM and FCS. ${ }^{160}$ Evidently, FCS is orthogonal to the AFM technique.

A combination of these two techniques was utilized typically in the analysis of liquid films and membranes. ${ }^{150,151}$ The first study utilizing AFM-FCS was done by Burns et al. in 2005. It was utilized to analyze the relative partitioning and the mobility of labeled probes in gel and disordered liquid phase of bilayers consisting of 1,2-dipalmitoyl-sn-glycero-3-phosphocholine (DPPC) lipid domains in 1,2-dioleoyl-sn-glycero-3-phosphocholine (DOPC). ${ }^{152}$ For their analysis, at first, sites with different domains were mapped by combined AFM and CLSM measurement, allowing the correlation of topography (AFM) with probe partitioning between gel and liquid domains (CLSM). The FCS analysis showed that two components are present in the DOPC fluid phase (fast and slow), which was ascribed to membrane heterogeneities. ${ }^{150}$ Subsequently, in the group of Schwille, the AFM-FCS technique was utilized to characterize dynamic and structural organization of phase-separated membranes ${ }^{161}$ and to show coexistence of the liquid disordered and liquid ordered phase for ceramides, which forms gel-like domains. ${ }^{153}$ The formation of these ceramide-rich domains was found to be dependent on the acyl chain length of the ceramide. ${ }^{151}$

2.2.5 AFM coupled with total internal reflection fluorescence (TIRF) microscopy. Total internal reflection fluorescence microscopy makes use of the total reflection of light, which happens at an excitation incidence angle greater than the critical angle, and the thereby originating evanescent field. Fluorophores near to the total reflection (glass surface) are selectively excited by interaction with the evanescent field. ${ }^{162}$ Fluorescence from these excited molecules can be collected by the microscope optics. The advantage of TIRF over a conventional CLSM/optical microscope and even super-resolution techniques (STED) is the high axial resolution of up to $100 \mathrm{~nm}$ (compared to approx. $500 \mathrm{~nm}$ in CLSM), which can be enhanced by multiangle or multiwavelength techniques up to approximately $20 \mathrm{~nm} .{ }^{162,163}$ Therefore, TIRF has applications for minute structures or molecules, which are located near to a surface (glass slide), as this technique enhances the signal to noise ratio. Such applications are for example surface nanobubbles, ${ }^{164}$ cell imaging ${ }^{165}$ and dynamics of membranes. ${ }^{166}$ Similar to a combination of CLSM and AFM, a combination of TIRF with AFM provides complementary benefits for sample characterization. The enhanced signal to noise ratio and the high sensitivity makes the TIRF-AFM combination especially useful for accessing high-resolution structural information of proteins or filaments, ${ }^{167}$ arrangement and orientation of these molecules, ${ }^{168}$ nanomanipulation ${ }^{169}$ and modulation (enhancement/quenching) of fluorescence similar to experiments with the CLSM. ${ }^{170}$

Many publications rely on the superior lateral and axial resolution of AFM over TIRF and the single photon accuracy of TIRF. ${ }^{168,171}$ These qualities were employed in the study of myosin labeled with tetramethylrhodamine-5-maleimide. ${ }^{168}$ Myosin self-assembled to asymmetric filaments. The crosscorrelation of AFM and TIRF (height/volume vs. intensity) yielded structural information of the assembly. The intensity versus height ratio implies that myosin heads form a shell around the filament axis, which is consistent with the heads exposed to the outside. ${ }^{168}$ Although TIRF is the technique with lower resolution, it was capable to detect the association of $\beta$-amyloidDOPC/ DPPC lipid bilayers earlier than AFM. ${ }^{171}$

AFM is often exploited for its force measurement capability and its ability to manipulate microscopic objects (micromanipulation) or even manipulation on an atomic scale (nanomanipulation) ${ }^{169,172-174}$ Such an application is the single cell manipulation via AFM to study the integrin Mac-1(cell)fibrinogen interactions in comparison to the intermolecular fibrinogen interactions. ${ }^{172}$ Force transmission, number, and arrangement of focal adhesion points of the cells can be analyzed by exerting a force on the apical membrane of cells, which adhere to a transparent and thin surface. ${ }^{173}$ Recently, TIRF was utilized to stimulate a single $\mathrm{T}$ cell by light (via photoactivable Rac protein) while simultaneously the mechanical response of the cell was measured via AFM. ${ }^{115}$ This method depicts an inverted approach of the AFM-TIRF setup, as typically the AFM is utilized as a nanomanipulator. Single cells could be seeded with fluorescent beads (fluorescent nanoparticles), which could be imaged subsequently with the TIRF setup. ${ }^{175} \mathrm{An}$ interesting approach to monitoring the genome release of adenovirus capsids was shown by Ortega-Esteban $e t$ al. In their research, similar to CLSM and FLIM essays for release studies, the rupture and release of fluorophores was observed by TIRF while the virus membrane was weakened and finally ruptured by the AFM (active trigger, see Fig. 5) ${ }^{174}$ They could show that the loss of the initial penton in frame 7 leads to a marginal incline in fluorescence but not to a reduction in height, whereas the rapid decrease of height after frame 17 signals the start of the complete disassembly, accompanied by a strong increase in fluorescence intensity. ${ }^{174}$ By advancing towards fluorescent nanoparticles/molecules, fluorescence modulation techniques similar to CLSM can be utilized. Eckel $e t$ al. used fluorescent CdSe nanocrystals and gold-modified AFM tips to enhance the emission, the tip-nanocrystal distance for maximum emission was $22 \mathrm{~nm}$, or to quench emission effectively. ${ }^{170}$ Here, the enhancement was attributed to resonant exciton-plasmon coupling. ${ }^{176}$ An enhancement of the previously mentioned method could be achieved by Lee et al. as they compensate for the photo brightening effect, which enables quantitative measurements. ${ }^{177}$ 
(a)

\subsection{AFM coupled to super-resolution techniques}

A further improvement of the AFM-CLSM combination was achieved by adopting super-resolution techniques, such as STED, ${ }^{179}$ FIONA, ${ }^{180}$ dSTORM ${ }^{181}$ and PALM. ${ }^{181}$ Such a combination has several advantages, amongst others, other fluorescentbased techniques can be adapted at least for the STED, such as FCS, ${ }^{182,183}$ FLIM, ${ }^{184}$ fluorescence recovery after photobleaching (FRAP), ${ }^{\mathbf{1 8 5}}$ multicolor imaging ${ }^{\mathbf{1 8 6 , 1 8 7}}$ and steady-state fluorescent probing. Via super-resolution techniques the lateral and axial resolution is enhanced up to $20 \mathrm{~nm}$ and $100 \mathrm{~nm}$, respectively, rendering subcellular structures observable in detail. Higher optical resolution was observed for non-commercial setups. Similar to an AFM-CLSM combination, the AFM-super resolution techniques benefit from the fast acquisition times of the optical method, although nowadays special AFM equipment is available with high temporal resolution. ${ }^{188}$ Therefore, via this combination tracking of several small molecules in close proximity, is possible (super-resolution technique) and simultaneous either nanomanipulation, ${ }^{\mathbf{1 8 9 , 1 9 0}}$ determination of change in morphology or viscoelastic properties of a sample. ${ }^{191}$ Similar to the CLSM, the chemical information/surrounding of a fluorophore can be evaluated, which proves to be difficult via the AFM. However, biomolecules and polymers are invisible to single-molecule localization microscopy (SMLM) as it only detects fluorophores with a specific absorption range and has to be stained. Furthermore, the z-resolution in SMLM methods is still in the order of 100-500 $\mathrm{nm}$, although advances in SMLM for example via a mirror-reflected approach yield in higher axial resolution. ${ }^{192}$ The successful combination of STED with AFM was first reported in 2012 by Harke et al. ${ }^{191}$ As the used experimental setup is similar to the setup for a combined AFM-CLSM experiment the representation of the setup was waived. AFMSTED was demonstrated at first for $40 \mathrm{~nm}$ fluorescing crimson spheres at dry and wet conditions. Subsequently, microtubules of Cos7 cells labelled with Atto $647 \mathrm{~N}$ were imaged and correlated to mechanical variations in the Young's modulus (see also Fig. 6). ${ }^{191}$ Chacko et al. showed that measurement artifacts of the optical image can be accounted for by AFM and the images can be corrected afterwards, i.e. by calibration via a Labview procedure. ${ }^{193}$ Furthermore, AFM subdiffraction nanomanipulation of fluorescent crimson microspheres visualizes the high-resolution targeting capability of the system. ${ }^{\mathbf{1 9 3}}$ Jianqiang et al. facilitated the combined setup by utilizing a super continuum laser and for the first time concomitant and synchronized optical and topographic images were obtained. They applied their setup for nanobeads with a resolution of $42 \mathrm{~nm}$ as well as cells. ${ }^{194}$ Recently, dSTORM and PALM were combined with the AFM to measure f-actin (dSTORM) and living mammalian cells (PALM). While the AFM images illustrate the dynamics of the cell membrane a PALM series monitors the changes in protein clusters over time. ${ }^{181}$ Similarly, 

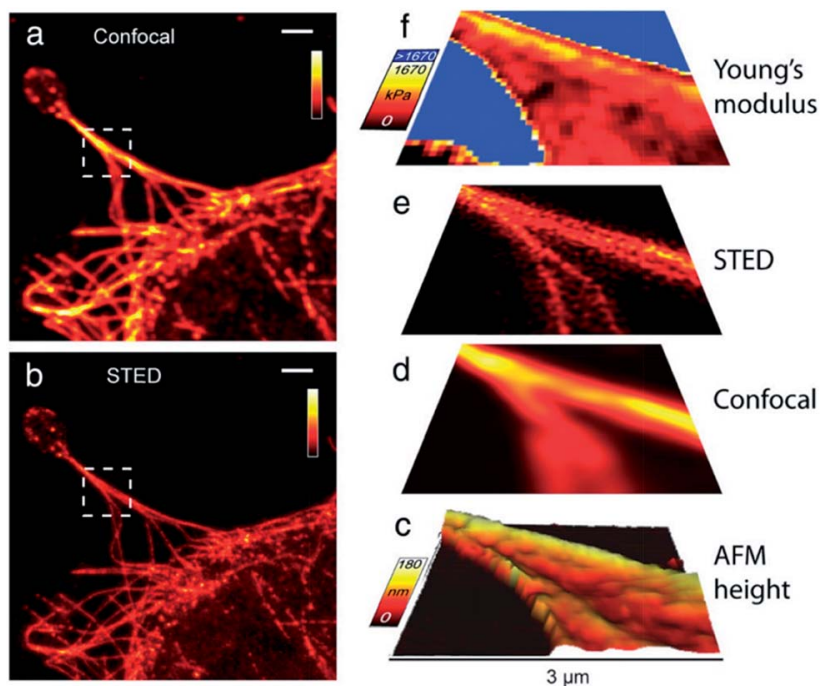

Fig. 6 Cos7 cells labeled with Atto $647 \mathrm{~N}$, (a) confocal image, (b) STED image, (c) 3D AFM height image extracted from AFM force curves (45 $\times 45$ pixilated scan), (d) confocal image and (e) STED image (both linear deconvolved) (f) map of Young's modulus calculated from AFM force curves. Scale bars in in (a) and (b): $2 \mu \mathrm{m}$, axes bars in (c)-(f): $3 \mu \mathrm{m}$. Reprinted from open-access article ref. 191 under the terms of the Creative Commons Attribution License.

a combination of AFM with FIONA was introduced by Fronczek et $a l .{ }^{195}$ It was utilized to identify different proteins in multiprotein-DNA complexes with a resolution of up to $8 \mathrm{~nm}$. Nowadays, commercial solutions for STED are available, which can be upgraded with FLIM and an AFM module. Therefore, these systems are expected to receive increased attention, especially in biological research areas.

\subsection{Near-field optical microscopy (SNOM/NSOM)}

The idea of SNOM was probably first conceived by Ash et al. in $1972{ }^{196}$ By use of $3 \mathrm{~cm}$ microwave radiation, a resolution of $\lambda / 60$ in one dimension and $\lambda / 20$ for two-dimensional objects was achieved, but technical difficulties prevented the extension of this technique towards shorter wavelengths. 2007 by Oshikane et al. the lateral and vertical resolution was determined to be 20 and 5-2 nm, respectively. ${ }^{197}$ SNOM is a nano-optical imaging technique that enables light-matter interaction for the purpose of extracting relevant information about nanoscale objects. There are two inherently different methods in scanning near-field optical microscopy. One can make use of a sub-wavelength aperture from which excitation light emanates or through which the emission from the sample is detected, or both. This is called aperture SNOM. The resolution in aperture SNOM is proportional to the radius of the aperture. However, as the aperture size decreases the amount of light admitted into the aperture decreases as well and signal levels drop. This results in a trade-off between signal levels and spatial resolution leading to a practical resolution limit for aperture SNOM of $\lambda / 10 .{ }^{198}$ The other method is executed by probing highly localized interactions of excitation and emission photons with a sub-wavelength structure. These structures are usually in the range of a few nanometers to a few tens of nanometers (aNSOM or NSOM). ${ }^{199,200}$ However, the contrast mechanism is not fully understood and images may contain topographical artifacts. ${ }^{201}$ Apertureless NSOM is typically measured via a bottom-illumination mode, ${ }^{202-206}$ which yields in advantages in light confinement and background noise reduction. As the probing tip has to be in close proximity to the glass surface, the measurement by aNSOM is restricted to thin samples, which diminishes its applicability. Recently, an aNSOM was developed with the capability to measure via bottom-illumination and top-illumination mode, as well as a measure in water and in air. ${ }^{207}$ The resolution was found to be independent on the illumination mode, but was lower in water $(32 \pm 7 \mathrm{~nm})$ than in air $(25 \pm 6 \mathrm{~nm}) .{ }^{207}$ Furthermore, the resolution can be enhanced by amplifying the incident and scattered electric fields at the tip itself. ${ }^{208}$ These results indicate that aNSOM in top-illumination mode (also called s-SNOM) is able to measure thicker samples, which are not measurable via the bottom-illumination mode. Both, the setup of apertureless and aperture NSOM are shown in Fig. 7, indicating the different approaches to enhanced resolution.

Nowadays, s-SNOM is coupled to IR to resolve intermolecular interactions with nanometer spatial resolution, ${ }^{\mathbf{2 1 0 , 2 1 1}}$ which is yet, similar to PTIR, another method to chemically identify surfaces with high spatial resolution. This field is under vast development and recently numerous research articles were published. $^{23212}$ For example, Pollard et al. investigated as a proof of principle the self-organization of amphiphilic block copolymers into hydrophilic and hydrophobic domains. By their approach, they were able to chemically map the surface with high spatial resolution while obtaining exquisite sensitivity, which can be used for the unravelling of intra- and intermolecular interaction (see Fig. 8). ${ }^{211}$ Still, SNOM methods are burdened with various artifacts, i.e. due to a broken or blunt tip. There is up to now no method to evaluate if an image is without an imaging artifact. ${ }^{213}$ As SNOM relies on the concomitant measurement of the optical and force microscopy, a fast and simple survey scan similar to typical optical microscopy is not possible anymore. On these grounds and because aperture SNOM is still often utilized (more artifacts, lower resolution compared to other methods) a combination of conventional optical microscopy may be favorable to SNOM and s-SNOM, especially for large three-dimensional samples (optical slicing ability). Applications of NSOM/SNOM and the novel s-SNOM are mostly biological appliances, such as cell visualization and analysis. ${ }^{\mathbf{2 1 4}}$ However, also applications in microscopy for materials are well known for example, analysis of semiconducting heterostructures, such as quantum dots, quantum wires and disordered quantum wells, material imaging, e.g. local crystal structures, crystal defects and doping, ${ }^{215}$ and observation of intra- and intermolecular interaction in both biological and material science fields. ${ }^{211}$

\subsection{AFM \& Raman spectroscopy - tip-enhanced Raman spectroscopy (TERS)}

Raman spectroscopy is the complementary technique to IR spectroscopy as most vibrations are either Raman or IR active, 
(a)

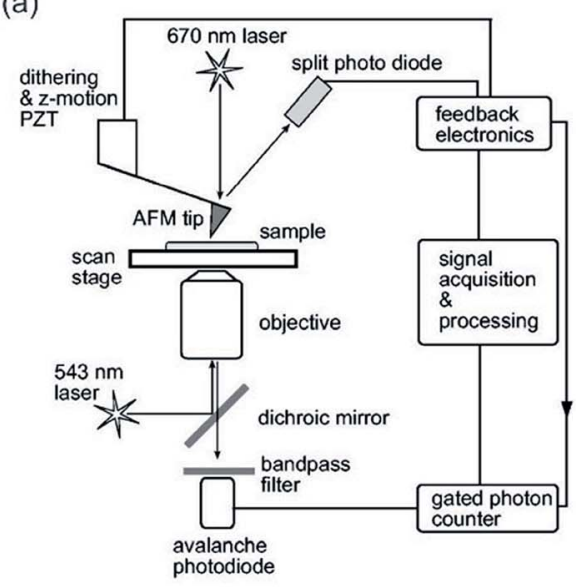

(b)

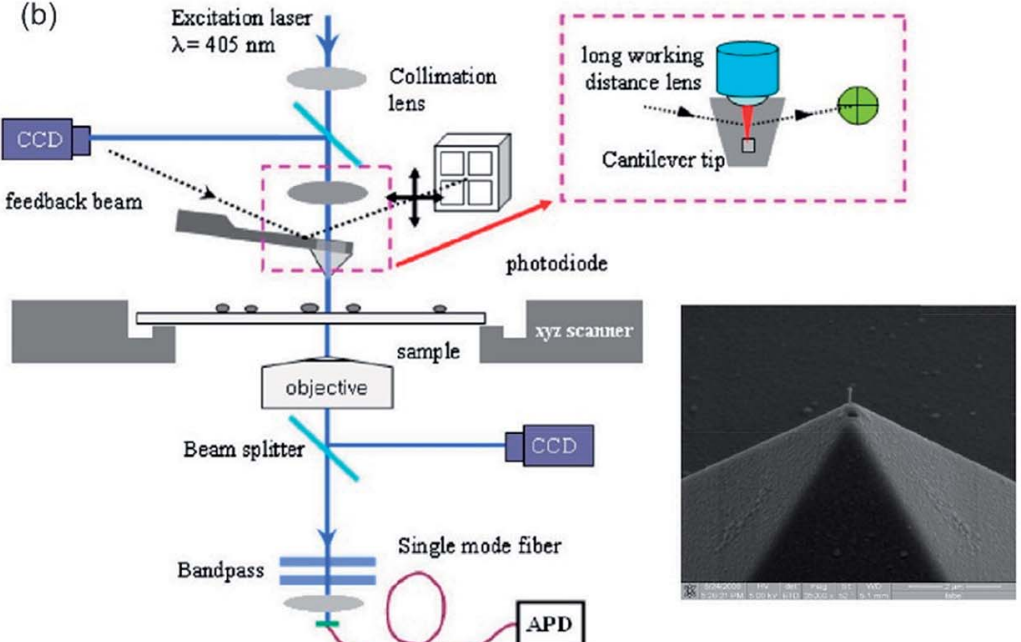

Fig. 7 (a) Schematic diagram of an apertureless NSOM. Reprinted with permission from, ${ }^{199}$ Copyright (2000) AIP Publishing. (b) Aperture SNOM, the inset shows the cantilever tip with its aperture. Reprinted with permission from, ${ }^{209}$ Copyright (2008) Elsevier.

and it is also orthogonal to AFM as Raman is able to gather chemical information of a desired sample. ${ }^{216,217}$ The former refers to the different selection rule compared to IR. Raman occurs mainly for symmetric vibrations when the polarizability changes upon excitation. Raman spectroscopy relies on the inelastic scattering of monochromatic light (nowadays laser),
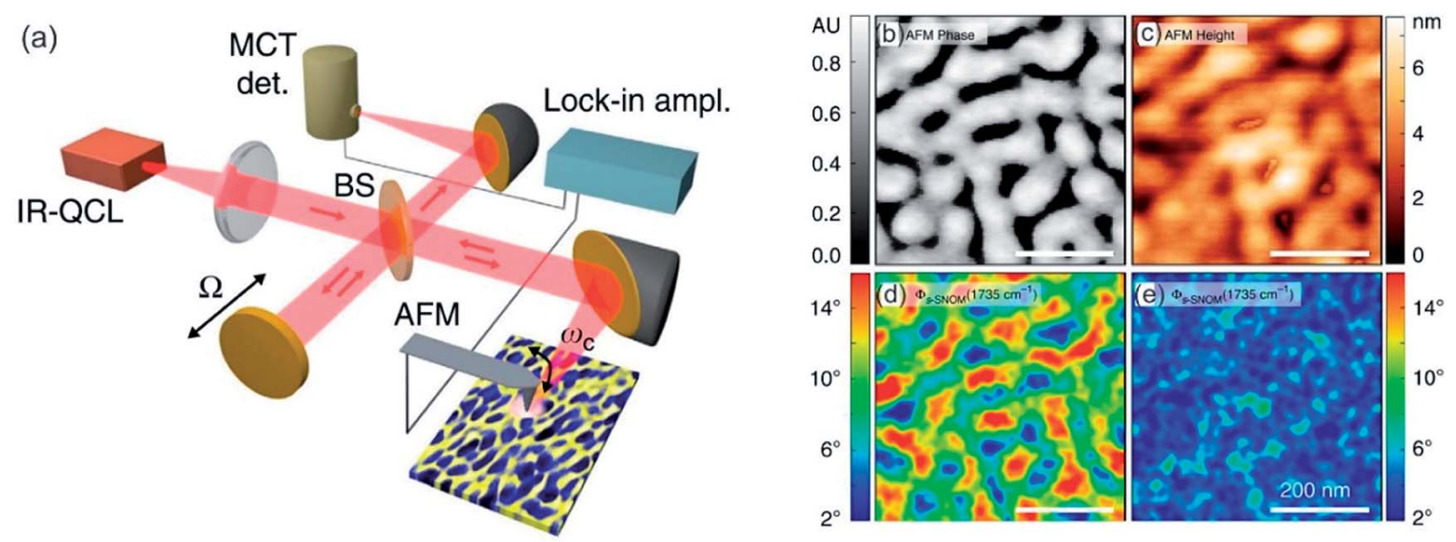

(f)

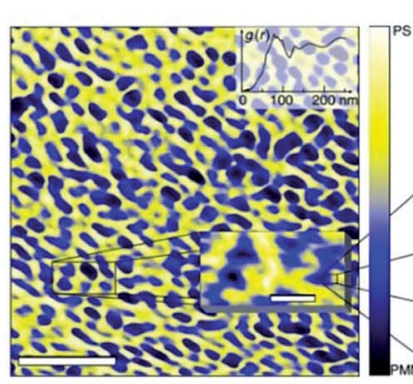

(g)

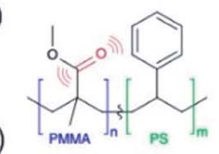

(h)

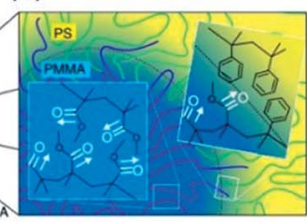

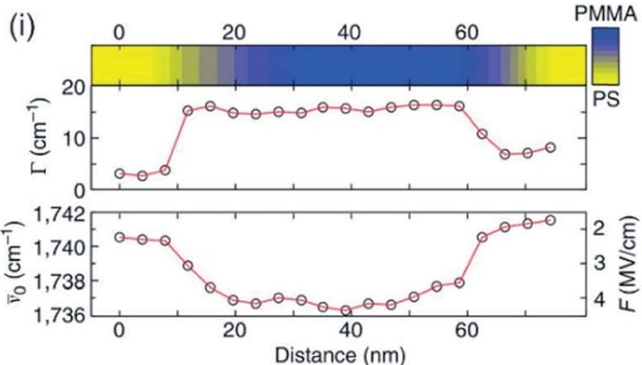

Fig. 8 (a) Scheme of interferometric s-SNOM experiment. QLC = quantum cascade laser, MCT IR detector = HgCdTe infrared detector, BS = beam splitter (b-e) sample scans of quasi-lamellar PS-b-PMMA showing AFM tapping phase (b), AFM height (c) and near-field phase (fs-SNOM) (d) and off resonance (e) with the carbonyl mode. Scale bars $200 \mathrm{~nm}$. (f) Nanoscale chemical map of self-phase separated amphiphilic block copolymer PS- $b$-PMMA derived from carbonyl resonant s-SNOM imaging. Scale bar $750 \mathrm{~nm}$. In the inset a magnification of a small ROI is shown (scale bar $100 \mathrm{~nm}$ ). (g) The molecular structure of block copolymer. (h) Schematic of domain morphology of PS-b-PMMA. (i) Spectrally resolved line cut showing the local relative concentration of PMMA as measured by the s-SNOM phase (top color map), linewidth (middle), and spectral shift (bottom), with derived local electric field F. Reprinted with permission from ref. 211, Copyright (2014) Macmillan Publishers Ltd., Nature Publishing Group. 
while the molecule (sample) is excited to a virtual energy state. From this state, an inelastically scattered photon emanates, which can possess either higher (anti-Stokes) or lower (Stokes) energy than the incoming photon. The difference in energy of the resulting scattered photon can be related to the energy differences in the rotational and vibrational states. Raman spectroscopy is widely utilized in chemistry and material science, ${ }^{218-221}$ as chemical bonds, functional groups and even different hybridization states are detectable. ${ }^{222,223}$ Especially, it is utilized in applications related to analytical chemistry, ${ }^{224}$ forensic science (analysis of drugs or paints), ${ }^{25,226}$ geology and mineralogy (e.g. gemstone identification), ${ }^{227}$ biology (e.g. secondary structure of polypeptides/proteins, phospholipids), ${ }^{228-231}$ material science ${ }^{232,233}$ (e.g. semiconductors, carbon materials and polymers) and life science (e.g. pharmaceutical analysis). ${ }^{234,235}$

Tip-enhanced Raman spectroscopy (TERS) combines the chemical sensitivity of surface-enhanced Raman spectroscopy (SERS) with the high spatial resolution of the AFM to concomitant garner chemical and morphological information at the nanometer length-scale. TERS is a near-field microscopy technique in which the Raman signal is enhanced by an apertureless probe. The probe is a sharp metal-coated AFM-tip, which is positioned at the center of a laser focus. The enhancement of the electromagnetic (EM) field and therefore of the Raman signal can be ascribed to a combination of localized surface plasmon (LSP) resonance and lightning rod effect. ${ }^{236}$ The enhancement effect is confined to the vicinity of the tip-apex. Hence, TERS overcomes the resolution limit conventional Raman spectroscopy is bound to. The resolution of TERS is typically in the order of $20 \mathrm{~nm}$ (see Fig. 9), ${ }^{231,237,238}$ but can be enhanced by specific reflection modes up to $1 \mathrm{~nm} .^{239}$

TERS has been utilized in a broad range of applications in biology, such as pathogens, ${ }^{241}$ cell membranes ${ }^{242}$ and peptides, ${ }^{243}$ in materials science, ${ }^{244}$ such as polymer blends, and chemical engineering, i.e. in catalysis ${ }^{238,245,246}$ for detection of intermediates or as a trigger for chemical reactions. ${ }^{247}$ For example, Kumar et al. investigated in situ photocatalytic oxidation of ( $p$-mercaptoaniline) $p \mathrm{MA}$ to $\left(p, p^{\prime}\right.$-dimercaptoazobenzene) DMAB on gold nanoparticles with TERS (see Fig. 10cf). Compared to far-field Raman spectroscopy, the integration time could be reduced while the peaks are more pronounced due to the enhancement effect in TERS, as shown in Fig. 10d and f. ${ }^{238}$ Due to the broad spectrum of TERS and its applications a thorough illustration of TERS would fill another review by its own. Therefore, we want to refer the interested reader to the reviews of Kumar et al. and Jiang et al., which summarize recent advances in TERS together with its advantages and weaknesses. ${ }^{240,248}$ TERS itself is developed further, even though it is already utilized for many applications. Important issues, which are encountered, are low fabrication reproducibility of the AFMtip, which becomes manifest in differing Raman enhancement factor or induced plasmonic dipole factors from tip to tip, ${ }^{249}$ short plasmonic lifetimes, which are assigned to metal delamination (Au-tips) or chemical alteration (Ag-tips) ${ }^{250,251}$ and low signal-to-noise ratio due to far-field scattering/emission. Extensive research was performed on solving these issues.
Improved fabrication reproducibility of the tip was achieved by utilizing an increased amount of wet-chemically synthesized silver nanowires (AgNWs), which are bound to the apex of the tip. This leads to improved electric contact, mechanical strength, and reproducibility, ${ }^{252}$ which can be exploited by the commercialization of such modified tips. However, TERS probes relying on $\mathrm{Ag}$-tips were found to lose the plasmonic enhancement of the tip during the first 4 hours of exposure to the environment (oxygen/humidity). It was shown that the lifetime of a TERS-tip based on Ag can be maintained up to 5 months by utilizing an environment with sub-ppm oxygen and moisture concentration (typically in a glovebox). It was also mentioned that $\mathrm{Ag}$ based tips can be protected for storage and measurement either by the inert atmosphere with previous mentioned low oxygen content and low humidity or by covering the tip with a thin $(\leq 3 \mathrm{~nm})$ layer of alumina. ${ }^{253,254}$ Furthermore, enhancement of TERS can be achieved by change of the illumination geometry, resulting in an even improved TERS signal, ${ }^{\mathbf{4 5}}$ which was observed for Raman spectra of malachite green isothiocyanate and thiophenol in water and in air (see Fig. 10a-f), and tip modification, which renders the combined setup sensitive to $\mathrm{pH}$ change. ${ }^{255}$

\subsection{AFM \& IR-spectroscopy - photothermal-induced resonance (PTIR) and infrared photoinduced force microscopy (IR-PiF)}

IR spectroscopy is one of the oldest and widespread analysis techniques for the analysis of solid samples. However, IR spectroscopy can be also utilized for liquids, ${ }^{256}$ soft matter ${ }^{257}$ and gases ${ }^{258}$ to identify chemical species. It is based on the absorption of light from the spectrum of a light source. Nowadays, IR is measured via the Fourier-transform technique also known as Fourier-transform infrared spectroscopy (FTIR), ${ }^{259}$ which enables fast and highly accurate measurement of whole spectra. Molecule vibrations, which change the dipole moment of the molecule, are IR active and may absorb light of a specific wavelength. This absorption of a specific wavelength can successively be translated into specific bonds of a molecule or related to small molecules themselves. Recently, the FTIR technique was enhanced by coupling it to an optical microscope. ${ }^{260}$ This results in FTIR microscopy with a lateral resolution of down to $10 \mu \mathrm{m}$ (compare diffraction limit). In an actual experiment, this value is with a scan size of $20 \times 20 \mu \mathrm{m}$ bigger, as it is difficult to obtain a good signal to noise ratio and enough IR radiation. ${ }^{261}$ The most important operation modes of FTIR are transmission, diffuse reflectance infrared Fourier transform (DRIFT) and attenuated total reflectance (ATR). ${ }^{262}$ The transmission mode can be utilized for gases, liquids, and solids, whereas the ATR method can only be utilized for liquids and solids. For a thorough explanation of FTIR and IR, its mathematics and its operation modes please refer to standard textbooks, e.g. "Fourier Transform Infrared (FTIR) Spectroscopy". ${ }^{263}$

IR and FTIR spectroscopy are widely utilized in biology/ biophysics (e.g. protein conformational changes), ${ }^{264,265}$ environmental science (e.g. gas analysis), ${ }^{26-268}$ chemistry (e.g. 

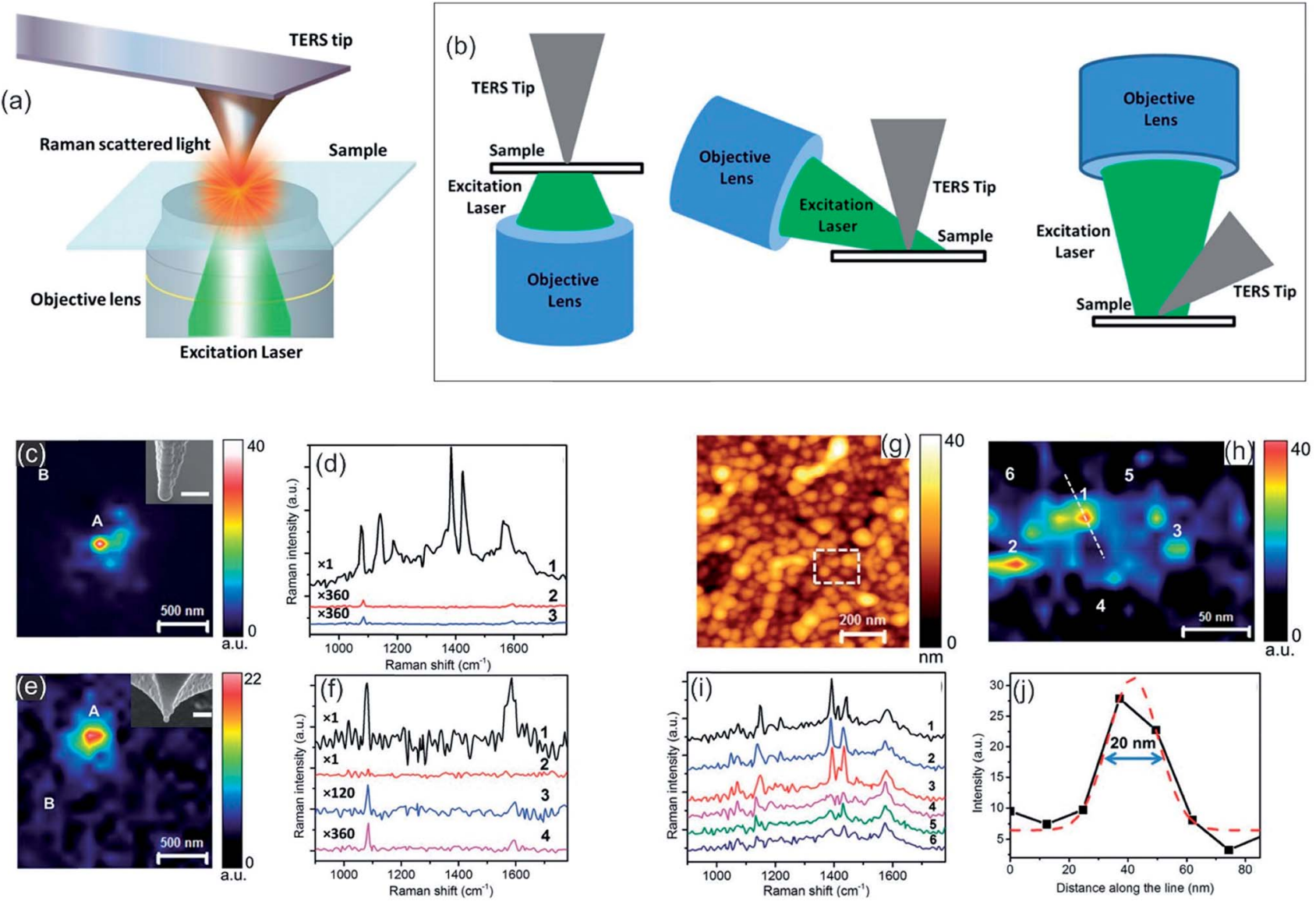

Fig. 9 (a) Schematic diagram of a TERS set-up in transmission mode. (b) Schematic diagrams of most commonly used TERS configurations. Bottom illumination (left), side illumination (middle) and top illumination (right). (a, b) Reprinted from open-access article ref. 240 under the terms of the Creative Commons Attribution License. (c) Mapping of photocatalytic reaction occurring at the Ag-coated TERS tip-apex using the $1142 \mathrm{~cm}^{-1}$ peak intensity of DMAB. In the inset, a SEM image of the tip is shown. Scale bar: $100 \mathrm{~nm}$. (d) Comparison of the near-field spectrum from position $A(1)$ in (c) with the tip in contact with the pMA/polymethylmethacrylat (PMMA) surface, its far-field spectrum (3) and far-field spectrum from position B (2) with the tip in contact with the surface. (e) Raman mapping of the alumina-protected Ag-coated TERS tip-apex using the $1086 \mathrm{~cm}^{-1}$ peak intensity of pMA. SEM image of the tip is shown in the inset. Scale bar: $100 \mathrm{~nm}$. (f) Comparison of near-field and farfield spectra of locations A and B from (e). (1) Near-field pos. A (2-4) far-field spectra. (g) AFM topography image of a glass substrate covered with Ag nanoparticles. (h) TERS map from the dashed rectangle marked in (g), showing the variation of the $1142 \mathrm{~cm}^{-1}$ Raman peak intensity of DMAB. (i) Near-field spectra from the positions marked in (h). (j) Intensity profile along the dotted line marked in (h), showing the spatial resolution of the TERS map. Gaussian fit to the intensity profile is shown by the dashed red curve. (c-j) Reprinted with permission from ref. 238, Copyright (2015) the Royal Society of Chemistry.

identification of molecules or polymers and chemical reactions in the gas phase) ${ }^{269}$ surface analysis ${ }^{270-274}$ (e.g. surface contamination, thin film analysis and analysis of surface termination) and polymer analysis ${ }^{275-277}$ (e.g. degree of crystallinity).

A combination of AFM with infrared spectroscopy or typically Fourier transform infrared spectroscopy is called AFM-IR, but often it is abbreviated PTIR (photothermal-induced resonance) due to its working principle. Dazzi et al. developed the concept of PTIR in $2010 .{ }^{278}$ PTIR relies on the excitation of molecules (vibration) by a tunable pulsed IR laser. The infrared light emitted from the pulsed IR laser is absorbed by the molecules leading to a quick thermal expansion. This expansion induces an oscillation of the AFM cantilever, which is detected via the deflection of the detection laser beam. By utilizing a Fourier transformation, frequencies and amplitudes can be acquired. Here, the obtained amplitudes are related to the strength of the cantilever oscillation. Studies utilizing PTIR have analyzed the chemical information of biological samples, such as proteins, ${ }^{279,280}$ lipids and lipid vesicles, ${ }^{281,282}$ cells, $^{283,284}$ bacteria, ${ }^{48,279,285}$ polymer samples and thin films, ${ }^{49,286-288}$ medical devices, ${ }^{289}$ drugs, ${ }^{290,291}$ nanostructures, ${ }^{292-294}$ metal-organic frameworks (MOFs), ${ }^{295}$ organo-halide perovskites, ${ }^{296,297}$ and nano-patterned metals ${ }^{298}$ at a resolution up to $50 \mathrm{~nm}$. Here the improved resolution of the PTIR serves to analyze nanoscale regions of relatively thin samples. The amplitude of the PTIR signal ( $S$, see eqn (9)) is dependent on the sample thickness $(z)$, absorbed energy per unit area $\left(U_{\text {abs }}\right)$, the thermal conductivity of the sample $(\eta)$ and thermal expansion of the sample $\alpha_{\exp }$ (see eqn (9)). The PTIR signal was found to increase linearly with thickness of the sample up to a thickness of $1 \mu \mathrm{m}$. Therefore, a sample for PTIR needs to possess a significant thickness of 4-15 nm. . $6,289,299^{-10}$ 

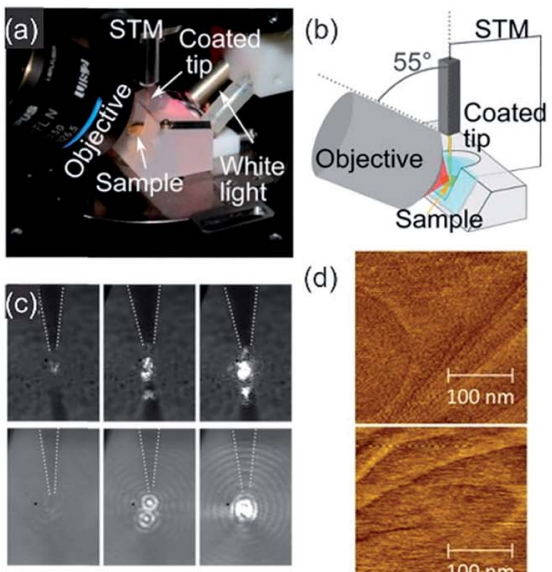

(d)

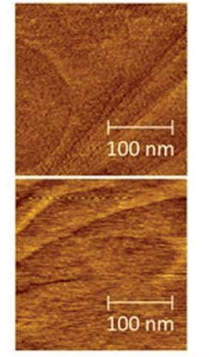

(e)

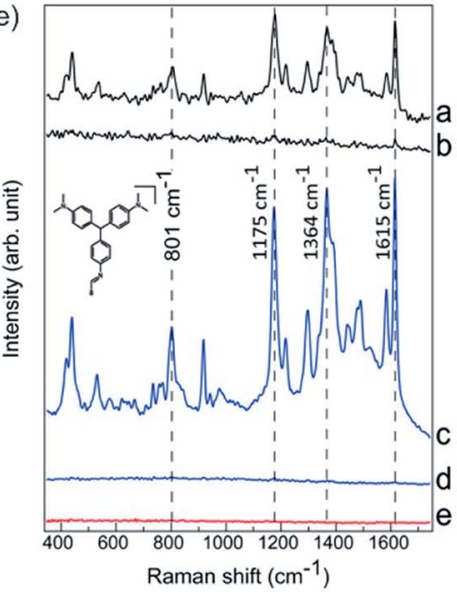

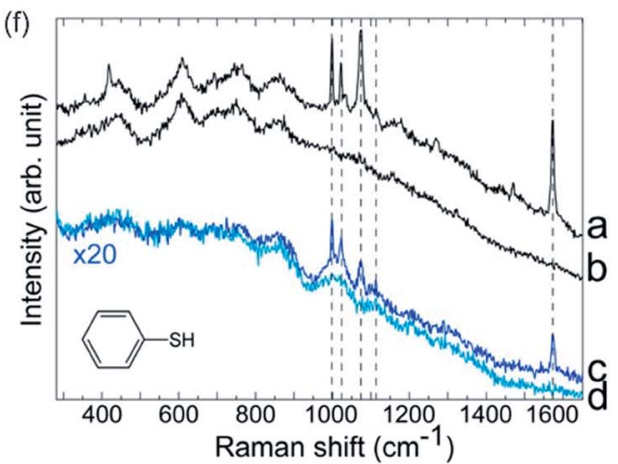

Fig. 10 (a) Experimental setup for solid/liquid TERS. (b) Scheme of the TERS setup (side illumination) (c) tip focusing process imaged in air (top) and water (bottom) (d) STM images of malachite green isothiocyanate (MGITC)/Au(111) in air (top) and in water (bottom). (e) Comparison of Raman spectra of MGITC Au(111) obtained via TER resonant (TERR) in air (e-a), far-field Raman in air (e-b), TERR in MilliQ water (e-c), far-field Raman in water (e-d) and signal of tip (e-e). (f) Comparison of Raman spectra of PhS/Au(111) obtained via TER resonant (TERR) in air (f-a), far-field Raman in air $(f-b)$, TERR in MilliQ water $(f-c)$, far-field Raman in water ( $f-d)$. Reprinted from open-access article ref. 45 under the terms of the Creative Commons Attribution License (CC-BY).

$$
S \sim \frac{\alpha_{\exp }}{\eta} \times U_{\mathrm{abs}} \times z^{3}
$$

The PTIR method was enhanced by utilizing resonance enhancement in 2014. ${ }^{299}$ The improved method relies on the $Q$-fold amplification of the signal, by matching the laser repetition rate of a quantum cascade laser to the resonance frequency of the cantilever oscillation. The oscillating cantilever senses the sample expansion and the absorbed energy is transferred to the cantilever yielding in an amplification factor $Q$ of approx. 100. This method is visualized in Fig. 11. Lu et al. utilized this specialized setup with a $p$-polarized pulsed midinfrared laser to induce photo expansion of polyethylene glycol methyl ether thiol (PEG) islands adsorbed on a gold modified mica surface. By this means, the $Q$-factor was enhanced to around 93 and thus, the sensitivity was estimated to 30 molecules of PEG with a thickness of $2 \mathrm{~nm}$. Via this method, a resolution of up to $25 \mathrm{~nm}$ was achieved (Fig. 11i) while even monolayers can be analyzed. ${ }^{299}$ Researchers have shown that polymers and self-assembled monolayers (SAMs) can be investigated via resonance enhanced-atomic force microscopy-infrared spectroscopy (RE-AFM-IR). ${ }^{\mathbf{2 9 9 , 3 0 0}}$ Recently, the coupling of mode synthesizing atomic force microscopy $\left(\right.$ MS-AFM) ${ }^{301}$ with PTIR was proposed, ${ }^{281}$ vesicles inside of bacteria could be detected and analyzed in situ. ${ }^{281}$ This method can potentially be generalized to nanocontainers inside soft matter.

Nowadays, PTIR is superseded by infrared photoinduced force microscopy (IR-PiFM) and recently, by peak force infrared microscopy (PFIR), ${ }^{302}$ both methods are related to PTIR. However, IR-PiFM is different from other spectroscopically sensitive force-detection technique as it can be operated in noncontact mode, generating high-resolution measurements in the sub-10 nm range ${ }^{303}$ while it can be conducted at ambient conditions without harmful contact between tip and sample, as shown in Fig. 12a. ${ }^{304,305}$ For example, Nowak et al. utilized this technique for chemical imaging of self-assembled block copolymer polystyrene- $b$-epoxydicyclopentadiene methacrylate (PS- $b$-PEDCPMA) patterns. Specifically, an incident mid-IR laser was employed to pulse at $f_{\mathrm{m}}=f_{1}-f_{0}$, where $f_{0}$ and $f_{1}$ are the first and second mechanical eigenmode resonances of the cantilever. The topography of the sample was recorded by the AFM feedback system at $f_{1}$, and the PiFM was concurrently recorded at $f_{0}$ by the feedback laser position-sensitive detector and lockin electronics. The sample was raster-scanned under the tip to generate the image. The incident light was polarized along the tip axis to maximize the signal coupling of the dipole-dipole force along the vertical direction of the cantilever vibration. High spatial resolution and chemical specificity were obtained by resolving and identifying each domain with $\sim 40 \mathrm{~nm}$ pitch (see Fig. 12b-i). ${ }^{304}$

Similarly, PFIR is capable of chemical imaging, a collection of infrared spectra, and mechanical mapping at a spatial resolution of $10 \mathrm{~nm}$. An advantage of this technique is the possibility to gather on top of chemical and topographical data also mechanical data, such as adhesion or moduli. ${ }^{302}$ Wang et al. showed its appliance, for example for the nanoscale phase separation in block copolymers. ${ }^{302}$

\subsection{AFM \& ellipsometry - scanning near-field ellipsometry microscopy (SNEM)}

Ellipsometry detects the changes of polarization of incident light while it is reflected from a surface. The polarization change is measured via the ellipsometry angles. These angles are related to parameters, such as thickness and the refractive index of the sample. ${ }^{306}$ The method is utilized to characterize 
(a)

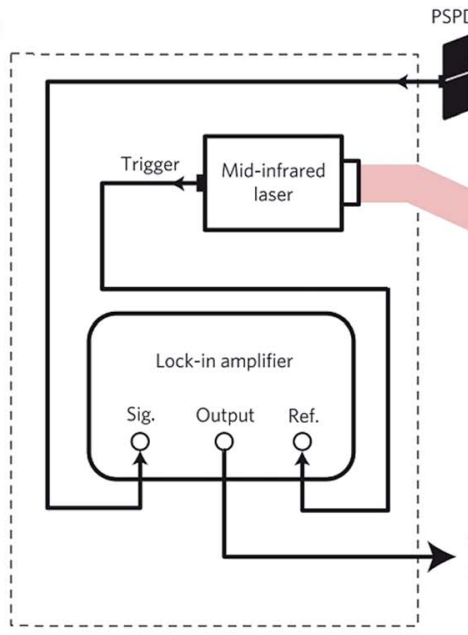

(d)

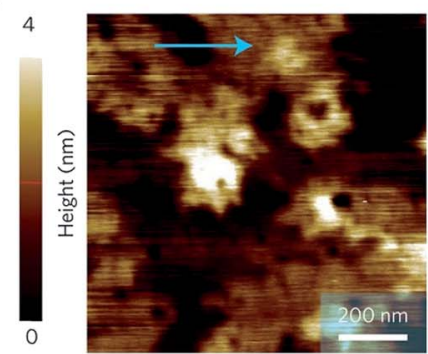

(h)

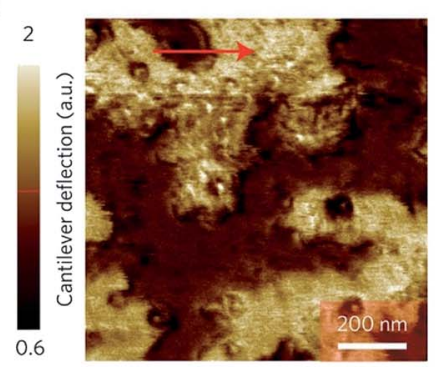

SPD
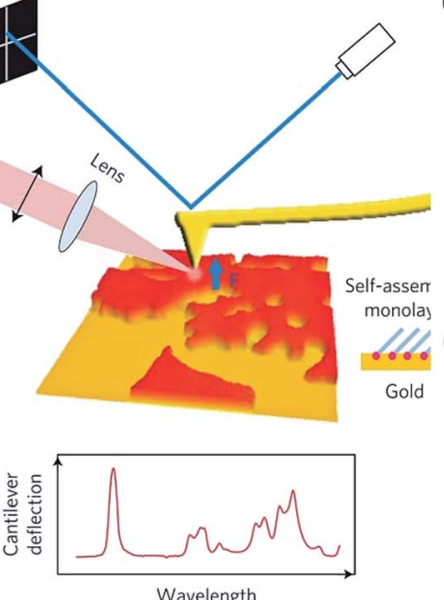

(e)

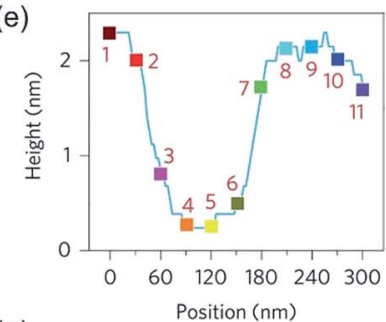

(g)

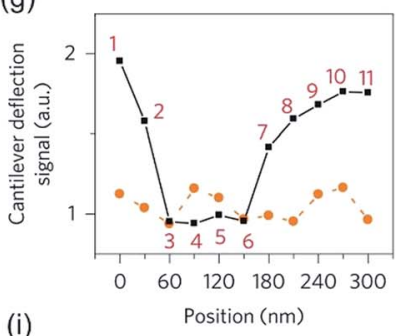

(i)

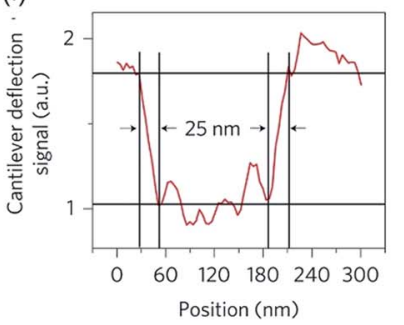

(b)

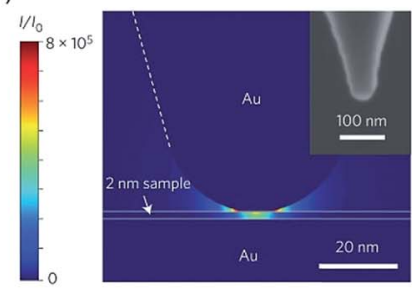

(c)

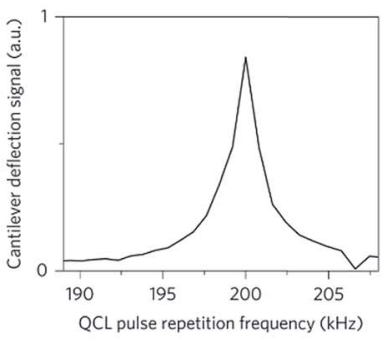

(f)

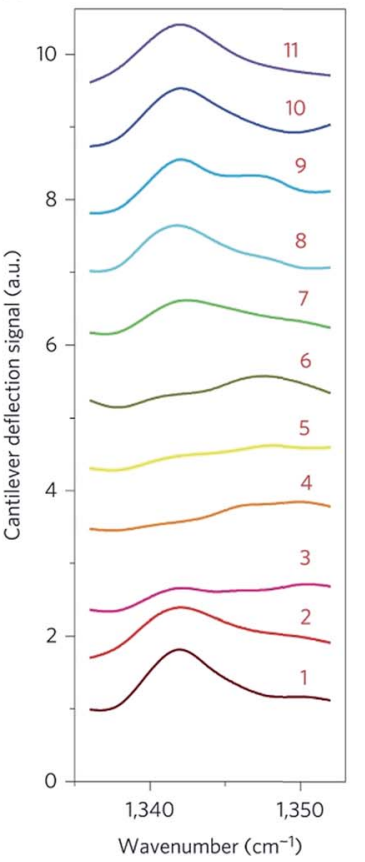

Fig. 11 (a). Schematic of the experimental set-up of PTIR. (b) 3D simulation of the tip-enhancement of the light intensity (I//O). For simulation, a molecular monolayer on gold with a thickness of $2 \mathrm{~nm}$, a mid-infrared light incident angle of $75^{\circ}$ to the surface normal and a geometry similar to the AFM tip was modeled with a radius of curvature of $25 \mathrm{~nm}$ and a half-cone angle of $17^{\circ}$. A SEM micrograph of the AFM tip is shown in the inset. (c) Experimental dependence of the lock-in output on the repetition frequency of quantum cascade laser pulses. (d) AFM topography image of PEG monolayer islands acquired in contact mode. (e) Line height profile along the blue arrow is shown in (d). (f) PTIR spectra taken at the positions indicated in (e). (g) Comparison of cantilever deflection signal between incident laser wavelength of $1342 \mathrm{~cm}^{-1}$ (PEG absorption line, black squares) and $1552 \mathrm{~cm}^{-1}$, no PEG absorption (orange circles). Data points were extracted from spectra in (f). (h) Mid-infrared mapping of monolayer islands at $1342 \mathrm{~cm}^{-1}$. Peg rich regions are bright, whereas at dark regions PEG is absent. (i) Signal along the line scan shown with a red arrow in (h), indicating a spatial resolution of $25 \mathrm{~nm}$. Reprinted with permission from ref. 299. Copyright (2014) Macmillan Publishers Ltd. (Nature publishing group).

material properties, such as composition, ${ }^{307}$ thickness (depth), ${ }^{308}$ crystalline nature ${ }^{309}$ doping concentration, ${ }^{310}$ electrical properties ${ }^{\mathbf{3 1 1}}$ and other material properties. AFM and ellipsometry were first combined in 2001, a scheme of such a combination is shown in Fig. 13a, to characterize concomitant optical (local refractive index) and topographical properties of thin polymer films. ${ }^{312}$ Via exploiting the electric field enhancement at a gold-coated AFM tip the signal to noise ratio (see Fig. 13b and c), as well as the resolution of the ellipsometry images, was enhanced by Tranchida et al., which was ascribed 
(a)
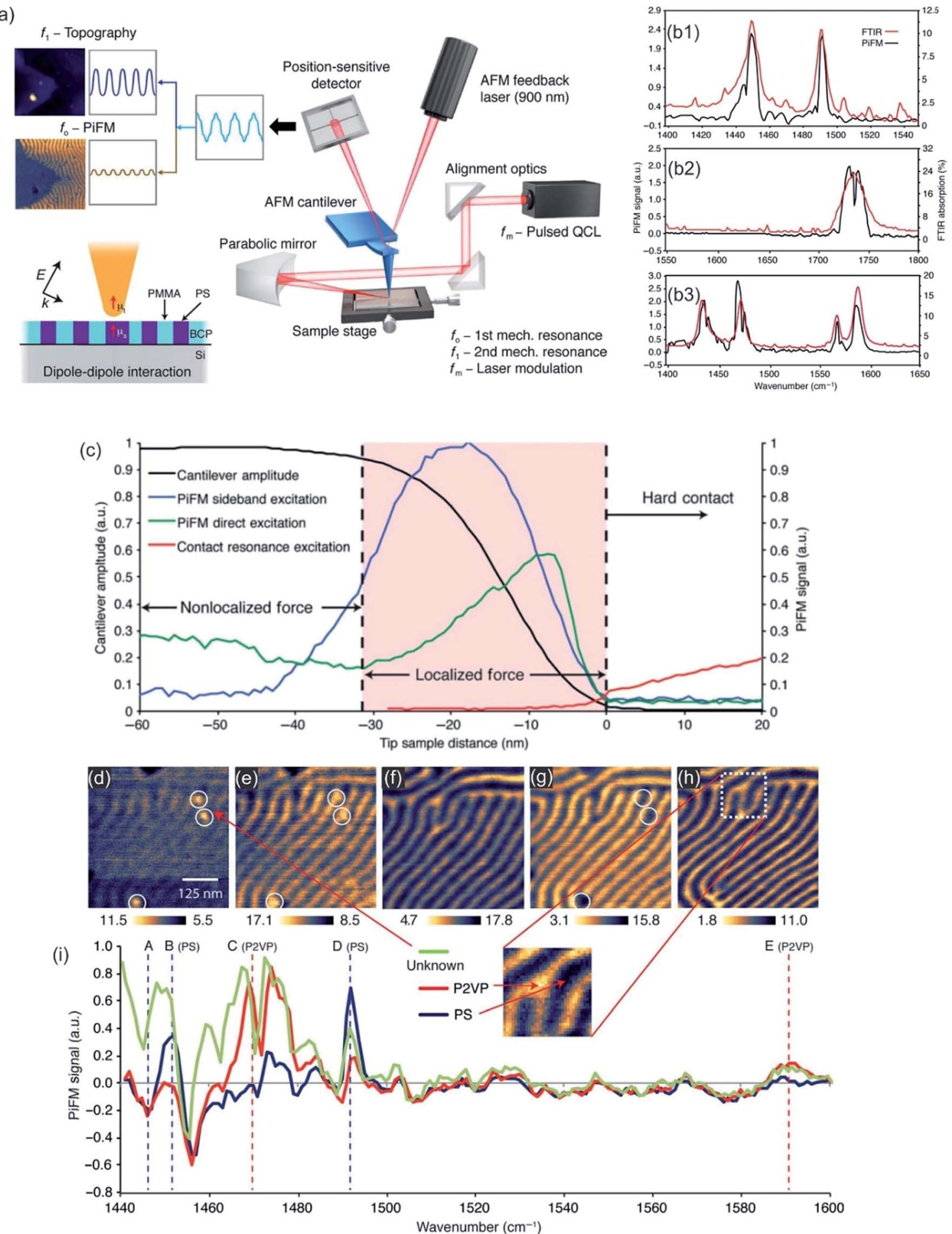

Fig. 12 (a) Schematic of IR-PiFM experiment. (b) Comparison of PiFM and FTIR spectra of homopolymers polystyrene (PS, b1), polymethylmethacrylate (PMMA, b2), and poly(2-vinylpyridine) (P2VP, b3). (c) The Pif signal and cantilever amplitude as a function of tip-sample distance on $10 \mathrm{~nm}$-thick PS- $b$-PEDCPMA brush prepared on Si and excited with $1733 \mathrm{~cm}^{-1}$. Fingerprint region of PS- $b$-P2VP. (d to h) PiFM imaging at (d) $1447 \mathrm{~cm}^{-1}$, (e) $1452 \mathrm{~cm}^{-1}$, (f) $1469 \mathrm{~cm}^{-1}$, (g) $1492 \mathrm{~cm}^{-1}$, and (h) $1589 \mathrm{~cm}^{-1}$. (i) Spectra were taken from point locations shown in (h), which were normalized against a Si background. Reprinted from open-access article ref. 304 under the terms of the Creative Commons Attribution License.

to lightning-rod effect and localized surface plasmons (see also TERS enhancement). ${ }^{313}$ The field enhancement was shown to be strongly distance dependent, denoting a significant effect at distances lower than $100 \mathrm{~nm}$. They were able to visualize concomitant with the AFM and the ellipsometry nanoparticles embedded in poly(methyl methacrylate) and microphases in 
(a)

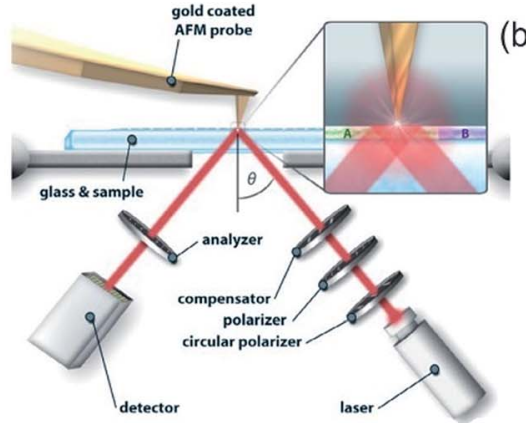

(b)

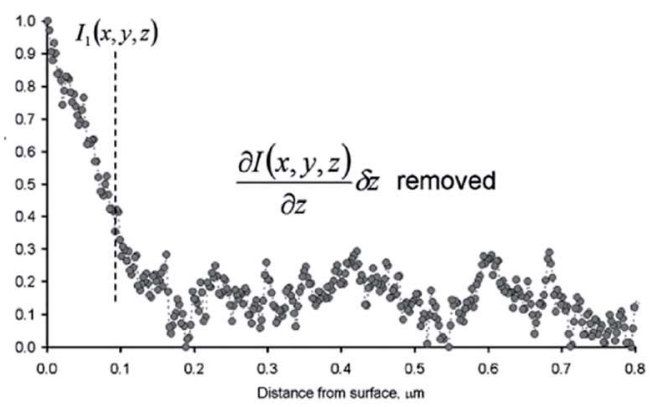

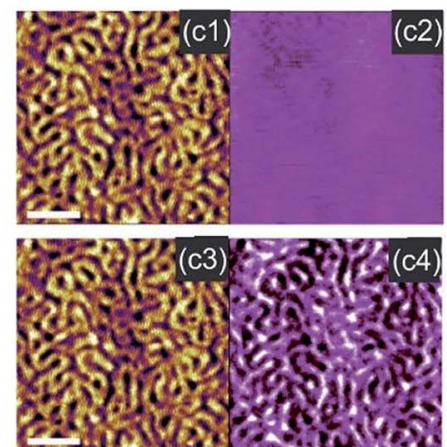

Fig. 13 (a) Scheme of the experimental setup of a SNEM experiment. The angle of incidence is fixed to around $\theta=40^{\circ}$. Reprinted with permission from, ${ }^{315}$ Copyright (2013) Elsevier. (b) Intensity vs. distance curve, obtained with a gold coated AFM tip during acquisition of a force-displacement curve on glass, corrected for the effect of background and interference. (c) SNEM data of micro-phase separated PS $2092-b$-PtBA ${ }_{1055}$ block copolymer film on glass: (c1, c3) AFM height image (the scale bar represents $500 \mathrm{~nm}$ ), (c2) optical image acquired without AFM tip in proximity of sample (the z-scale covers $0-12 \mathrm{mV}$ ) and the corresponding tip-enhanced SNEM optical image (c4). (b, c) Reprinted with permission from ref. 313 Copyright (2011) the Royal Society of Chemistry.

microphase-separated block copolymer films (vide Fig. 13c). For the enhancement effect, a tip with a gold surface is needed. Gold garners atmospheric contaminants and therefore a steady degradation in performance is observed in SNEM as well as TERS. Cumurcu et al. proposed a protective layer of ethanethiol (self-assembled monolayer), which yields consistent and reproducible results for scanning near-field ellipsometry microscopy for at least 5 days (storage at ambient conditions). ${ }^{\mathbf{3 1 4}}$ The combined SNEM method was advanced by Cumurcu et al. to a concomitant tapping mode SNEM measurement, which was applied for microphase-separated morphology of polystyrolblock-poly(2-vinylpyridine) (PS- $b$-P2VP) block copolymer thin films, reducing the inflicted damage originating from contact of the tip with the surface. ${ }^{315}$

\subsection{A combination of AFM with reflection interference contrast microscopy (RICM)}

The method RICM was invented in the 1960s to study the interaction of cells with a glass substrate. ${ }^{316}$ It is an interferometric technique that allows the determination of the vertical distance and of the contact area between the interfaces. ${ }^{317}$ RICM is a label-free method (similar to IR, Raman, and ellipsometry) and can be implemented into a standard inverted microscope with little investment. A RICM setup comprises out of a monochromatic light source, an inverted microscope equipped with an anti-flex objective and a CCD camera. The light beam passes an aperture diaphragm (AD) and a field diaphragm (FD), which is positioned with respect to the sample and objective to obtain a Köhler illumination. This illumination method generates an extremely even illumination. Subsequently, the light is linear polarized, reflected by a semi-reflecting mirror and passed through a quarter wave plate, which renders the light circularly polarized. The light is transmitted and reflected by the glass substrate and the sample (i.e. a cell). The reflected light of different distances from the glass surface (i.e. membrane a few nanometers away from the glass surface) interfere with polarized light reflected directly at the glass surface, which leads to constructive or destructive interference. The interference pattern is subsequently recorded as a two-dimensional matrix of intensities known as Newton rings. Out of these rings, the distance between two surfaces can be calculated by eqn (10).

$$
I(x)=I_{1}+I_{2}+2 \sqrt{I_{1} I_{2}} \cos (2 k h(x)+\pi)
$$

here, $k=2 \pi n / \lambda, n$ is the refractive index, $\lambda$ the wavelength of the monochromatic light, $h(x)$ is the separation distance between the two surfaces and $I_{1}$ and $I_{2}$ are the intensity of light reflected from the upper glass surface and the surface of the bead, respectively. A conceptional setup of a coupled measurement of AFM and RICM is shown in Fig. 14a.

In a combination with AFM, RICM can be a powerful tool, as it can assess the contact area of an AFM-tip with the surface/ polymer membrane. Therefore, it is a pivotal technique to study inter-surface interactions. The significance of the combination was boosted by advent of soft colloidal probes in AFM measurements, ${ }^{318}$ as by RICM the actual contact area is accessible, ${ }^{319}$ which further improved the sensitivity, for example for adhesion measurements.

Typically, the position of an interface can be detected easy and precise by an AFM. Hyaluronan brushes and other soft materials, i.e. hydrogels, possess in their swollen state a soft matter/water interface, which is difficult to locate by AFM. At this interface, an analysis of mechanical parameters (elasticity or viscosity) is difficult. Attili et al. utilized a combination of colloidal probe AFM and RICM to determine forces and concomitant the distances between a probe and an interface. ${ }^{320}$ The compressive mechanics of films of the polysaccharide hyaluronan that was end-grafted to a supported lipid bilayer via a biotin anchor was investigated. The compressive response of the polymer brushes was in good agreement with a theoretical model of polymer brushes. Furthermore, via RICM linear instrumental drifts of the AFM can be corrected, as well as cantilever sensitivity was obtained without additional reference measurements. ${ }^{320}$

Instead of compression, adhesion of a soft colloidal probe (SCP) was investigated by Schmidt et $a l .{ }^{321}$ The SCP was covered 
(a)

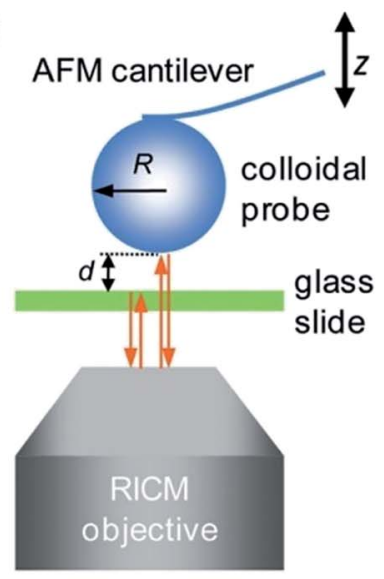

(b)

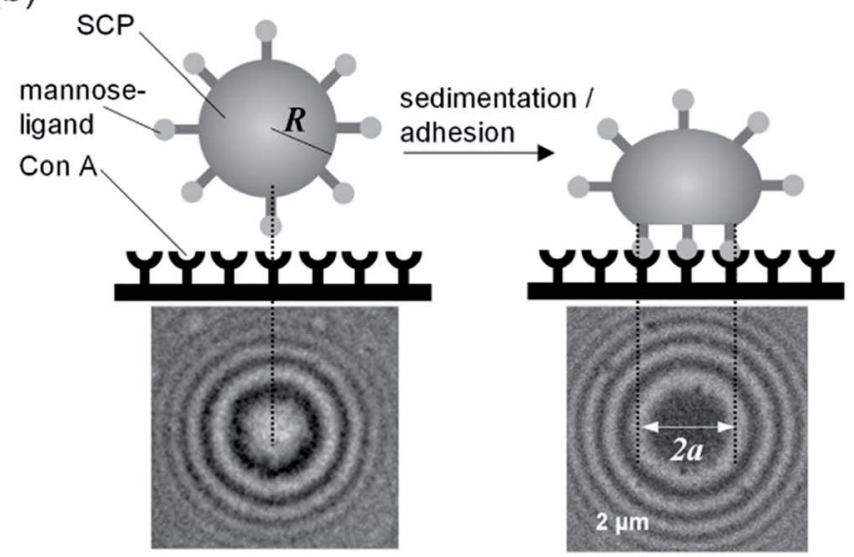

(c)

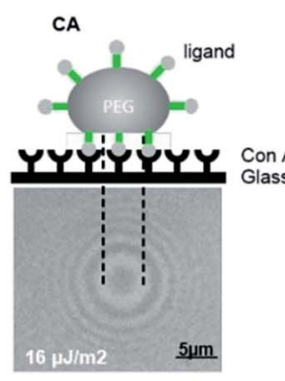

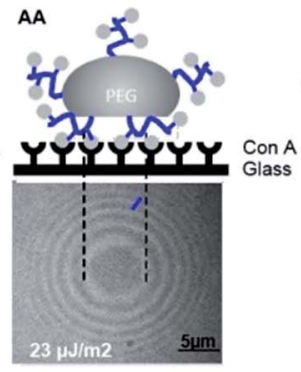

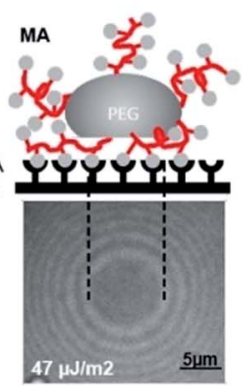

(d)

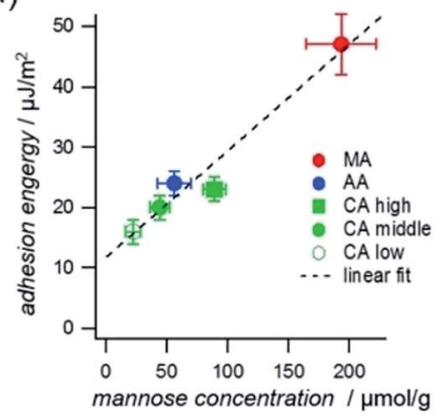

Fig. 14 (a) Combined colloidal probe AFM/RICM setup. Reprinted with permission from ref. 320. Copyright (2012) American Chemical Society. (b) SCP adhesion measurement sketch (top): a mannose-functionalized PEG-SCP sediments onto a Concanavalin A (ConA) receptor functionalized surface (left). Binding of mannose units to ConA (right). Central circular interference minimum (bottom, denoted by 2a). (c) Scheme of SCP deformation of polyethylenglycol-crotonic acid (PEG-CA) particles, PEG-MA (MA = methacrylic acid) and PEG-AA (AA = acrylic acid). Below, the corresponding interference patterns are presented. (d) Plot of the adhesion energy vs. mannose concentrations for SCPs with grafting type from (c) and PEG-CA SCPs with varying density of grafting units. Reprinted from open-access article ref. 321 under the terms of the Creative Commons Attribution License.

with a mannose ligand, which binds under contact to a surface covered with Concanavalin A (ConA) receptors (see Fig. 14b and c). The interaction energy between the SCP and the protein surface could be evaluated as the surface area is accessible via RICM by analysis of the extent of the central circular interference minimum. A linear relationship between the mannose density and adhesion energy was found (vide Fig. 14d), excluding affinity differences between mannose units. Therefore, multivalent binding types, such as chelate and subsite, were ruled out. ${ }^{321}$

\subsection{AFM coupled to surface plasmon resonance (SPR)}

With surface plasmon resonance (SPR) adsorption of molecules onto metal interfaces can be determined. ${ }^{322}$ SPR is a label-free method, ${ }^{323}$ possesses a high sensitivity, ${ }^{324}$ is able to monitor changes in adsorption in real-time, ${ }^{324}$ consumes low sample volumes $^{325}$ and is a quantitative measurement. Therefore, determination of quantitative kinetic rate constants is feasible (i.e. adsorption or desorption rate constants). ${ }^{326,327}$ In SPR the Kretschmann configuration ${ }^{328}$ is often utilized, which is shown in Fig. 15a. The Kretschmann configuration comprises of a thin metal film (silver or gold) at the interface of two dielectric media. A laser beam is focused onto a prism with refractive index $\left(n_{1}\right)$. The light is, dependent on the angle of incident, either reflected or adsorbed (resonance angle). The resonance angle changes upon the refractive index of the solution of interest, for example by adsorption. For the measurement, the solution of interest needs to possess a lower refractive index $\left(n_{2}\right)$. The surface plasmon resonance angle shift, which is originating from the change in refractive index, is utilized to determine the quantitative adsorption of molecules. SPR and AFM were already combined in 1995 by Chen et al. and Shakesheff et al. ${ }^{329,330}$ As the two systems are geometric complementary (AFM measured from the top of the sample, whereas SPR measures from bottom) a combination of these two methods is fairly easy. The time resolution of the SPR together with the high sensitivity can substitute in a combined AFM-SPR measurement the lack of time resolution (for most AFM equipment) of the AFM. Early studies have analyzed the degradation of biodegradable polymer films on silver. The combination can make use of synergy of both capabilities, as AFM can determine 
(a)

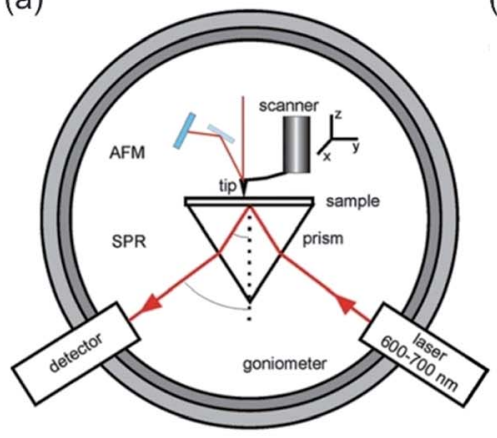

(b)

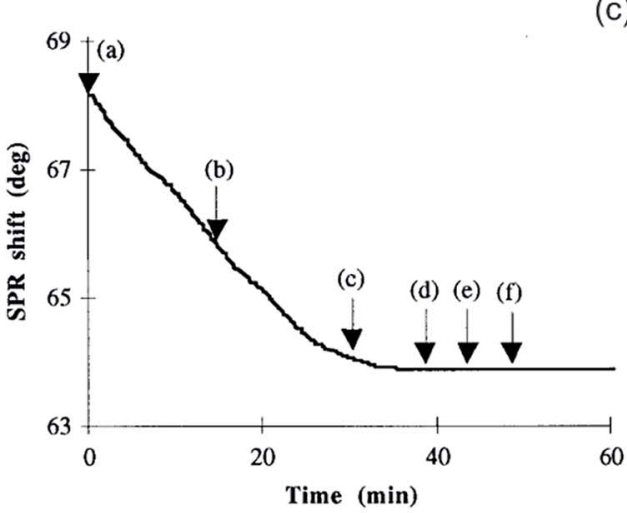

(c)

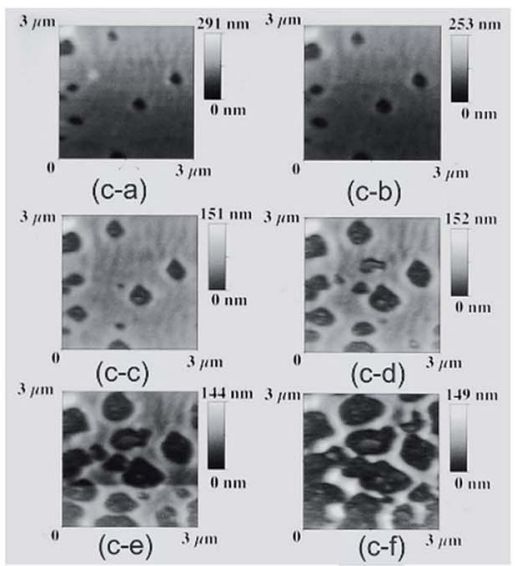

Fig. 15 (a) Instrumental configuration for AFM + SPR. Reprinted with permission from ref. 52 Copyright (2009) Royal Society of Chemistry. (b) Time dependence of SPR angle shift during degradation of a poly(orthoester) (POE) film exposed to flowing (1 $\mathrm{ml} \mathrm{s}^{-1}$ ) acidic solution ( $p H$ 3.5). The arrows indicate the acquisition of corresponding scanning force microscopy (SFM) scans (c) at the time $t=0 \mathrm{~min}$ (c-a), $t=15$ min (c-b), $t=$ $29 \mathrm{~min}(\mathrm{c}-\mathrm{c}), t=39 \mathrm{~min}(\mathrm{c}-\mathrm{d}), t=44 \mathrm{~min}(\mathrm{c}-\mathrm{e})$, and $t=49 \mathrm{~min}$ (c-f). (b, c) Reprinted with permission from ref. 334 Copyright (1996) AIP Publishing LLC.

structural changes of the polymer films during the degradation, whereas SPR determines the thinning of the polymer film, as shown in Fig. 15b and c. ${ }^{329}$ For conjugated polymer electropolymerization, a combined in situ AFM-SPR-electrochemical analysis yields in surface morphological properties and domain growth of the film by AFM, whereas via SPR \& electrochemical analysis the electrochemical behavior depending on the polymer is accessible. ${ }^{331}$ Similarly, both techniques have been employed for analysis of mucoadhesion of pectins ${ }^{332}$ and for real-time measurements of changes in the mammalian membrane mimics structure induced by antimicrobial peptides. ${ }^{333}$ For the latter, a detailed model for antimicrobial peptide action as a function of the degree of bilayer disruption was proposed by a comparative AFM, SPR, and polarization interferometry (DPI) analysis. ${ }^{333}$

\section{Combination of AFM with non- optical surface sensitive methods}

3.1 Combination of AFM with Kelvin method - Kelvin probe force microscopy (KPFM)

By rendering the AFM tip and the sample conductive, the work function or surface potential can be obtained with a high lateral resolution, which can be lower than $10 \mathrm{~nm} .{ }^{335}$ The method is commonly denoted as Kelvin probe force microscopy (KPFM) and was first introduced in 1991 by Nonnenmacher et al. ${ }^{336}$ KPFM measures the contact potential difference (CPD) of a conductive AFM tip and a conductive sample. CPD is defined by the work functions of the tip ( $\left.\Phi_{\text {Tip }}\right)$ and the sample $\left(\Phi_{\text {Sample }}\right)$ and the elementary charge $(e)$.

$$
V_{\mathrm{CPD}}=\frac{\phi_{\text {sample }}}{e}-\frac{\phi_{\mathrm{tip}}}{e}=\frac{\Delta \phi}{e}
$$

By applying both, an $\mathrm{AC}\left(V_{\mathrm{AC}}\right.$ also $\left.U_{\mathrm{AC}}\right)$ and a $\mathrm{DC}\left(V_{\mathrm{DC}}\right.$ also $\left.U_{\mathrm{DC}}\right)$ voltage to the AFM tip, the work function of the sample is assessed. Here, the DC voltage repeals the CPD difference between tip and sample surface, which is oscillated due to the applied AC voltage. The cantilever oscillates at $a \sin (\omega t)$ (sinusoidal excitation signal). $a$ denotes for the amplitude and $\omega$ for the frequency of the sinusoidal electrical signal. The feedback controller $\mathrm{C}$ compensates for the surface potential $\Phi$ indicated by ++++ by adjusting a DC (bias) voltage $U_{\mathrm{DC}}$, as shown in Fig. 16a. ${ }^{337}$ As a consequence of these applied signals, the resulting force has three contributions, two oscillatory terms $\omega$ and $2 \omega$ and a bias, which contributes to the topographical signal. The contact potential difference can be obtained by a lock-in technique by tuning the bias voltage $U_{\mathrm{DC}}$ to a value, which nulls the oscillating force signal $(\omega)$. The contact potential difference is this needed bias voltage.

The surface potential can be determined by at least 4 methods. Here, the KPFM is the most versatile and possesses distinct advantages over standard Kelvin probe (KP), photoemission spectroscopy (PES) and scanning electron microscopy (SEM). The measurement principle of KP and KPFM is similar. The energy resolution of KP method is slightly better compared to KPFM, but KP method relies on averaging over the whole sample, indicating no or low lateral resolution is obtainable. Via PES both, lateral resolution of approximately $100 \mathrm{~nm}$ and 20 meV energy resolution can be obtained. ${ }^{339}$ However, KPFM possesses enhanced lateral and energy resolution. However, for a sample surface containing adsorbents, KPFM cannot distinguish between surface band bending and surface dipoles created by adsorbents from the sample surface. ${ }^{340}$ PES yields in the spectral distribution of the surface potential, which can be utilized in a comparative approach to determine band bending and surface dipole contributions, independently. ${ }^{340}$ A qualitative measure of the surface potential can be obtained with a lateral resolution of approximately $70 \mathrm{~nm}$ by SEM via electron beam induced current. ${ }^{341}$ KPFM has an energy resolution of 5$20 \mathrm{meV}$ and a lateral resolution of $10 \mathrm{~nm}$ or better, ${ }^{335}$ depending on the utilized measurement mode. 
(a)

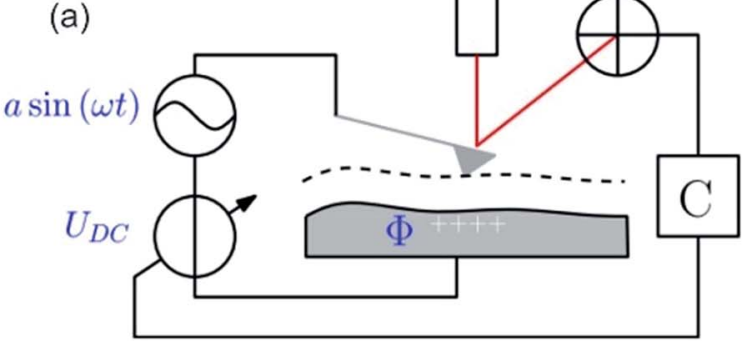

(b)

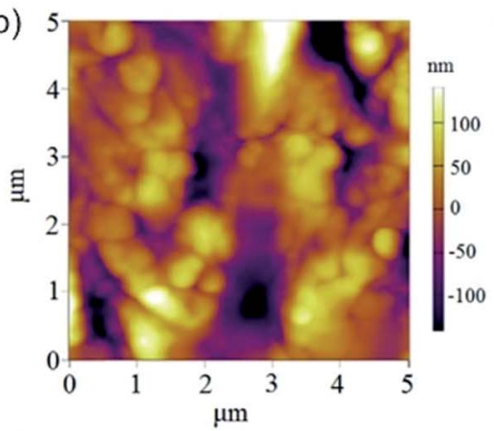

(e)

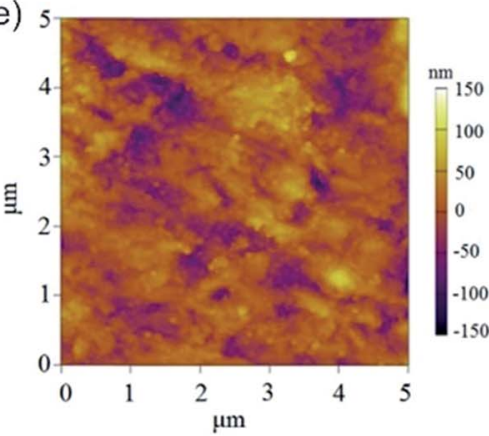

(c)

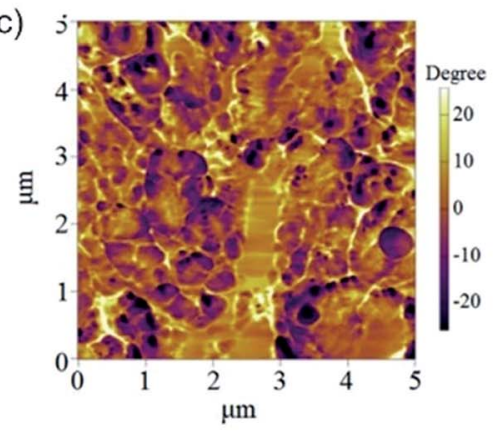

(f)

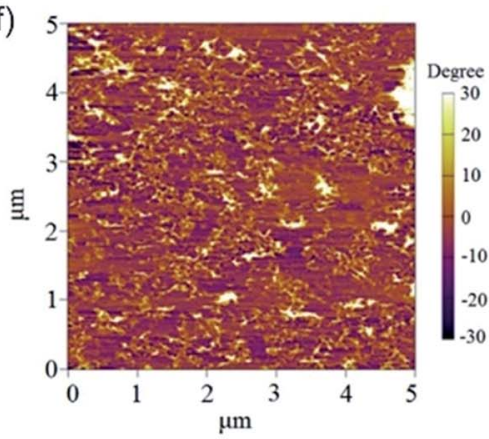

(d)

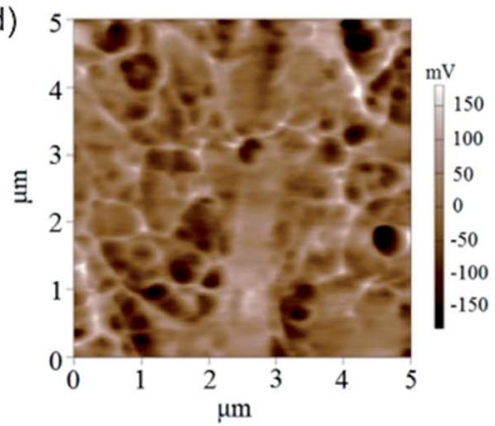

(g)

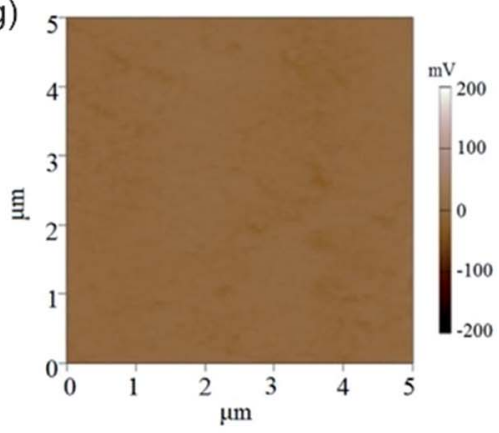

Fig. 16 (a) Schematic illustration of the Kelvin probe force microscopy setup. Reprinted from open-access article ref. 337 under the terms of the Creative Commons Attribution License. (b-d) AFM and KPFM of Al-6061 cv 3\%. (b) AFM height, (c) AFM phase and (d) KPFM potential mapping of Al-6061 covetic 3\% sample. $V$ can be computed by inverting and scaling the signal by one half $(V=-2 b)$ (e-g) AFM and KPFM of pure Al-6061. (e) AFM height (f) AFM phase and (g) KPFM potential mapping of a pure Al-6061 sample of the same region. Reprinted with permission from ref. 338 Copyright (2016) Elsevier.

Typically, a KPFM experiment is conducted in either an amplitude modulation mode (AM) or frequency modulation mode (FM). Here, FM-KPFM is considered the method with higher lateral resolution (up to sub-nanometer), whereas AMKPFM possesses a higher energy resolution of up to $5 \mathrm{meV}$. The enhanced $V_{\mathrm{CPD}}$ precision can be related to a higher signalto-noise ratio originating from the resonance peak of the cantilever, whereas in FM mode the $V_{\mathrm{CPD}}$ is determined by FM demodulator, which increases the noise in a measurement. ${ }^{342}$

Ongoing research is devoted to enhancing the time resolution, because the scan speed of FM mode is $\mathrm{slow}^{343}$ and AM mode measurements are burdened with artifacts, such as the stray capacitance effect ${ }^{344}$ and interfering signals, leading to topographical coupling. ${ }^{345,346}$ Several approaches try to couple the lateral resolution and reproducibility of FM mode KPFM with enhanced time resolution similar to AM mode KPFM, such as time-resolved electrostatic force microscopy, pump-probe KPFM, ${ }^{347}$ general acquisition mode, ${ }^{348}$ open loop (OL) KPFM $^{349}$ and Heterodyne (H) KPFM. ${ }^{350}$ For example, H-KPFM improved the scan rate while maintaining the spatial resolution and voltage sensitivity comparable to FM-KPFM. ${ }^{59}$ Furthermore, it is not susceptible to artifacts such as stray capacitance (AM mode), topographical oscillation (FM mode) and AC inductive coupling (AM mode) artifacts..$^{59,346}$ However, artifacts originating from collisions with the surface persist.

KPFM is applied for characterization of metal ${ }^{351}$ and semiconductor surfaces, ${ }^{352}$ organic films ${ }^{353}$ and biological membranes. ${ }^{354}$ Surface potential mapping is utilized for distinguishing carbon from the alloying elements, such as $\mathrm{Al}, \mathrm{Zn}$ and $\mathrm{Mg}$, as the surface potential difference is relatively small for carbon (similar work function as the tip (Pt)), as is illustrated in Fig. $16 \mathrm{~b}-\mathrm{e} .{ }^{338}$ In contrast, the potential difference between the metals and tip are high. ${ }^{338}$ For example, Jaim et al. utilized KPFM to analyze the difference between Al-6061 cv 3\% and Al6061 and substantiated the presence and the ribbon networks of carbon embedded in the Al-matrix of Al-6061 cv 3\%. They 
observed a correlation of the dark regions in the AFM phase image (Fig. 16b) and KPFM image (Fig. 16c), which was ascribed to carbon as it possesses a lower work function compared to the Al-matrix surface potential. Consequently, Al-6061 does not show this correlation between AFM phase and KPFM image, which appears to possess a rather homogeneous CPD. Another common area of application of KPFM is the analysis of semiconductors. ${ }^{352,355,356}$ Here, the KPFM method is sensitive enough to determine the dopant concentration in the range of $10^{14}$ $\mathrm{cm}^{-3}$ to $10^{19} \mathrm{~cm}^{-3} \cdot{ }^{356}$ However, the contact potential difference is altered by surface defects, ${ }^{357}$ exploitable for detecting of defects. ${ }^{358}$ By illumination with a sub-bandgap laser, band bending is reduced and higher contrast in CPD in conjunction with reduced influence on surface defect states is achieved. ${ }^{356}$ KPFM can be applied for Langmuir-Blodgett films of organic molecules on water (subphase) to assess the packing of the organic molecules at varying surface pressure by means of the CPD. ${ }^{353}$ Furthermore, KPFM can be utilized to ascertain charge transfer from Pt adsorbates to $\mathrm{TiO}_{2}$ surface ${ }^{359}$ and analysis of charge separation for the benchmark of organic solar cells, which for example consist of poly(3-hexylthiophene) (P3HT)/ fullerene (C60) acid-methyl ester (PCBM). ${ }^{155}$ An interesting application, which also shows the high lateral resolution and sensitivity, is the analysis of quantum size effects (QSE) proposed by Späth et al. In contrast to other techniques, KPFM was able to directly address the single digit nanometer height of single $\mathrm{Pb}$ islands on $\mathrm{Si}$ while simultaneously assessing the local work function (see Fig. 17). ${ }^{360}$ Yamagishi et al. utilized KPFM to visualize trapped charges in organic thin-film transistor (OTFT) channels. ${ }^{361}$ KPFM was utilized in the biological area to examine
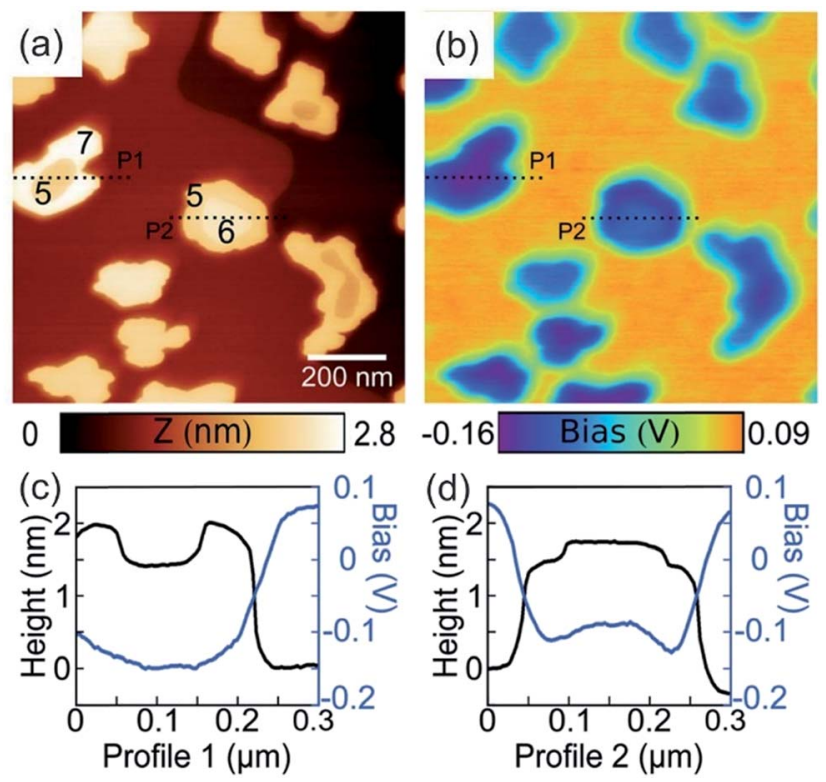

Fig. 17 SFM topographic (a) and simultaneously obtained Kelvin images (b) of ultra-thin Pb islands on Si(111). The local height in atomic layers measured from the wetting layer is indicated by the numbers in the images. ( $c$, d) Profiles over $\mathrm{Pb}$ islands along the dashed lines marked in (a, b). Reprinted with permission from ref. 360 Copyright (2017) Royal Society of Chemistry. the effects of surface potential on microbial adhesion on various metals. The surface potential of the microbial was dependent on adsorbents and was linked with changes in cellular metabolism and motility. ${ }^{60}$

\subsection{AFM combined with quartz crystal microbalance (QCM)}

QCM is a method to detect and quantify change of the mass by a shift in the frequency of vibration of a piezoelectric device. ${ }^{362}$ The frequency shift ( $\Delta F$, eqn (12)) of the resonator is dependent on the fundamental frequency of quartz resonator $\left(F_{0}\right)$, change of mass on the surface of quartz resonator $(\Delta m)$ and the vibrating surface area $(s)$.

$$
\Delta F=-2.3 \times 10^{6} F_{0}{ }^{2} \frac{\Delta m}{s}
$$

In addition to the detection of mass change, it may provide information about the conformation of molecules adsorbed to the surface by damping of the oscillation (amplitude). The latter information is often hard to come by, as changes in shape cannot directly be related to a QCM response. On the other hand, it is feasible to analyze changes in shape by AFM. Therefore, it is conducive for analysis of QCM data. For a combined measurement, the oscillation amplitude has to be lower than the desired resolution of the AFM experiment. The first combination was reported in 1998 by Iwata et al. and was used to evaluate the morphology and mass change at the liquid/ solid interface during the electrodeposition of $\mathrm{Ag}$ on a Pt thin film. ${ }^{363}$ For electrochemistry purposes, a specialized threeelectrode cell was utilized, comprising of an Au coated quartz crystal working electrode (WE), Ag wire reference electrode (RE), and $\mathrm{Zn}$ foil counter electrode (CE) (see Fig. 18a). Such a setup was successful in performing in situ electrochemistry, as shown in Fig. 18b-d. ${ }^{363-365}$ For example, Smith et al. were able to confirm that the growth mechanism of electrodeposited $\mathrm{Zn}$ resembles a 3D progressive model. ${ }^{365}$ Thus, this hybrid method imparts a realistic prospect of understanding of electrochemical nucleation and growth, which may be transferable to other issues outside electrochemistry. Most recently, Kelvin probe microscopy was annexed to an AFM-QCM combination to improve the versatility. ${ }^{366}$ This approach was utilized to measure the response of tin oxide-based sensors and determine during adsorption of analyte in situ the work function of the sensor nanomaterial (i.e. $\mathrm{SnO}_{2}, \mathrm{Pd}$-doped $\mathrm{SnO}_{2}$, and bromocresol purple-doped $\left.\mathrm{HfO}_{2}\right) \cdot{ }^{366}$

\subsection{AFM combined with thermal imaging - scanning thermal microscopy (SThM)}

Scanning thermal microscopy (SThM) maps the local temperature and thermal conductivity of interfaces. SThM allows thermal measurements at the nano-scale. Besides temperature and energy transport, processes involving the exchange of energy and entropy with the surroundings are feasible, such as thermal properties of materials, ${ }^{367}$ thermal conductivity, ${ }^{50}$ heat capacity, glass transition temperature, ${ }^{368}$ latent heat, enthalpy, etc. 
(a)

In 1986, shortly after the scanning tunneling microscope was invented, Williams and Wickramasinghe reported a nanoscale measurement exploiting thermal phenomena. ${ }^{369}$ Several scanning thermal microscopes were established, which are mainly based on atomic force microscopy. Here, the combined system with the AFM diversifies the applicability, for example by performing measurements as a function of the tip-sample force and distance ${ }^{370,371}$ or by varying sensor types and geometries. The working principle of SThM is dependent on the utilized thermal method. The methods can be classified according to the temperature-dependent mechanism into either (a) thermovoltage, ${ }^{372-374}$ (b) change in electrical resistance, ${ }^{375-377}$ (c) fluorescence $^{378}$ or (d) thermal expansion. ${ }^{379,380}$ However, these methods are still under rapid development and other techniques have been proposed, for example a method based on simultaneous registration of the static and the dynamic electrical resistance of the probe driven by the sum of DC and AC currents, which is suitable for thin films deposited on thick substrates. ${ }^{\mathbf{3 8 1}}$ Reprinted with permission from ref. 365 Copyright (2009) American Chemical Society.

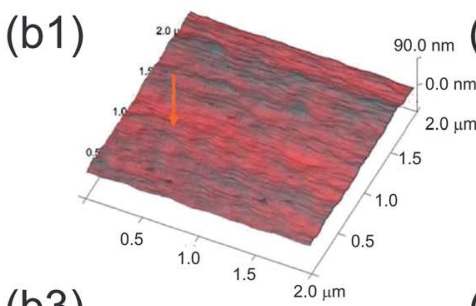

(b3)
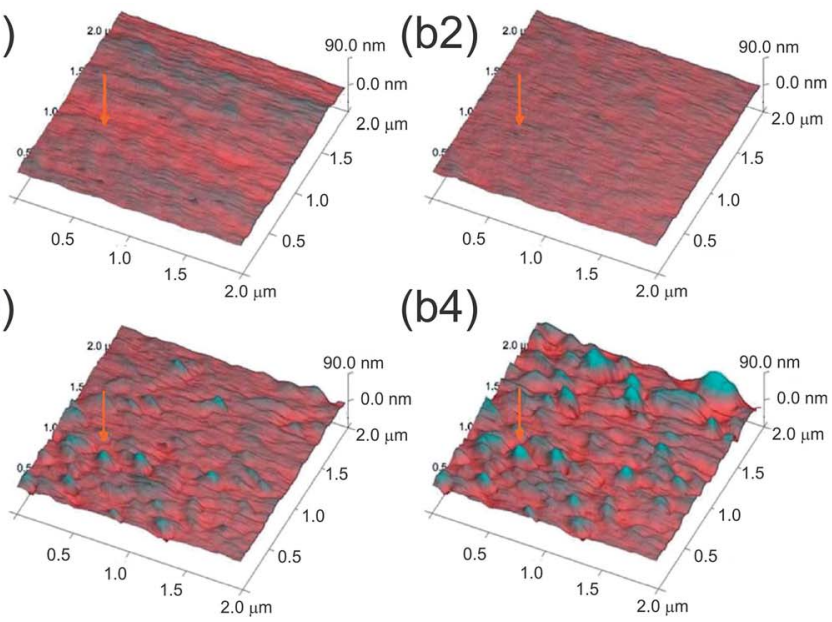

(b4)

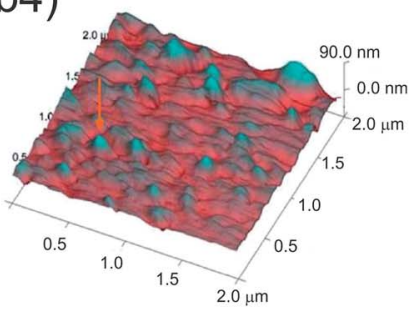

(d)

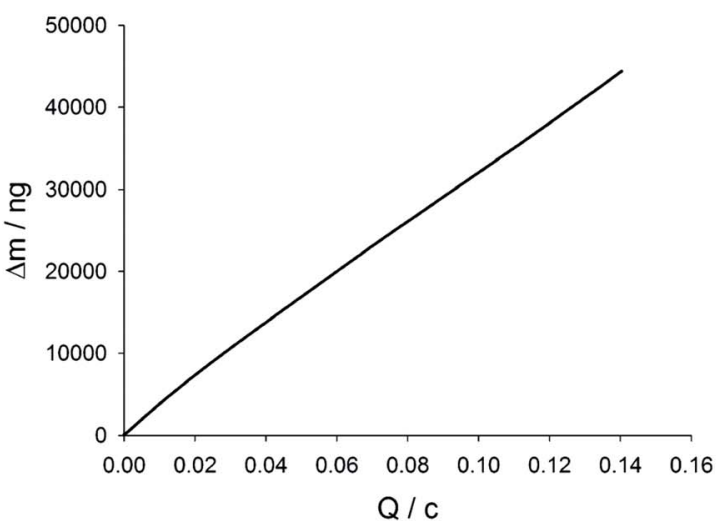

Fig. 18 (a) Schematic of a custom-built AFM-QCM hybrid especially designed for electrochemistry, also called electrochemical QCM. (b) Tapping mode, AFM images recorded in liquid at a concentration of $0.3 \mathrm{M} \mathrm{ZnCl}_{2}$ in ethaline of an Au coated resonating quartz crystal under electrochemical control ( $-1.1 \mathrm{~V}$ versus Ag wire). Height images show Zn deposition at times (b1)t $=0 \mathrm{~s}(\mathrm{bare} A \mathrm{Au}),(\mathrm{b} 2) t=120 \mathrm{~s},(\mathrm{~b} 3) t=240 \mathrm{~s}$ and no baseline correction. (d) Deposited mass, $\Delta m$, versus passed charge (Q), acquired simultaneously with AFM images during deposition.

(a) Thermovoltage based measurements can be conducted either in contact or in non-contact mode. Crucial, but also limiting its applicability is the need for an electrically conducting surface of the sample. The working principle of thermovoltage methods can be tunneling thermometry ${ }^{382}$ or the point-contact thermocouple method. ${ }^{383}$ As probes in thermovoltage-based methods, thermocouples (see Fig. 19c) ${ }^{374,384}$ and Schottky diode are utilized. ${ }^{385,386}$ Accessible via these methods are the temperature and the thermal conductivity ${ }^{387,388}$ at a minuscule surface area. The lateral resolution utilizing a thermovoltage method is in the $\mathrm{nm}$ range and both the lateral resolution and probe thermal time constant of an experiment was substantial enhanced by miniaturization of the cantilever, the tip and the junction at the tip. ${ }^{374,384}$ Thermovoltage methods are per se not quantitative due to the dependence between temperature rise measured locally on the size of the heated area (heat transfer also via gas), as shown in Fig. 19b. Vacuum conditions (below 0.1 Pa) with an active thermal feedback scheme allows maintaining the tip temperature equal to the sample surface temperature. ${ }^{389}$ 
(a)

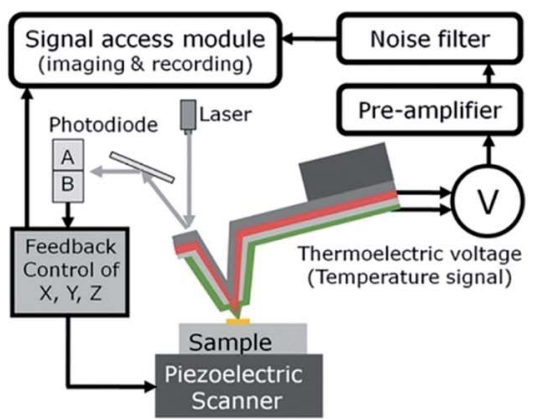

(c)

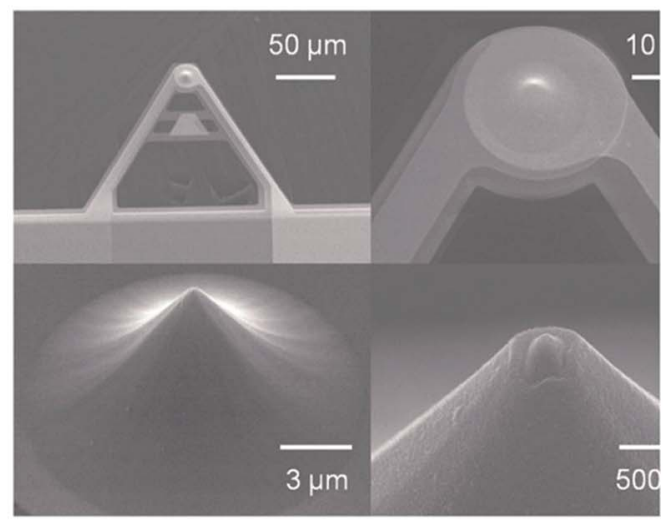

(e)

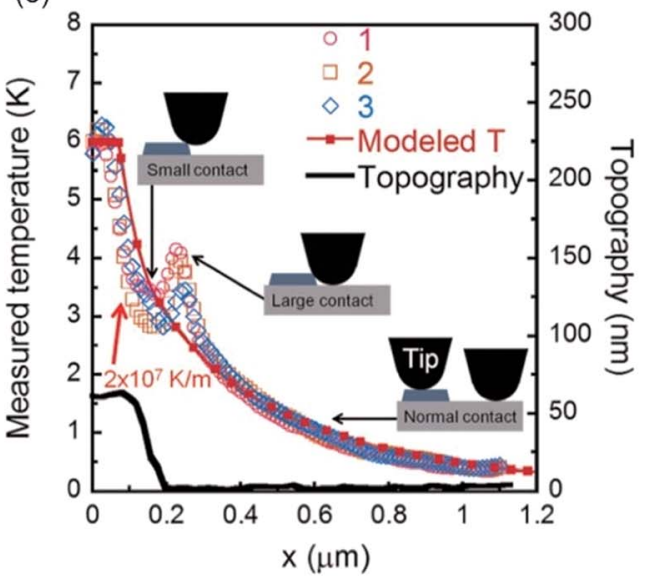

(b)

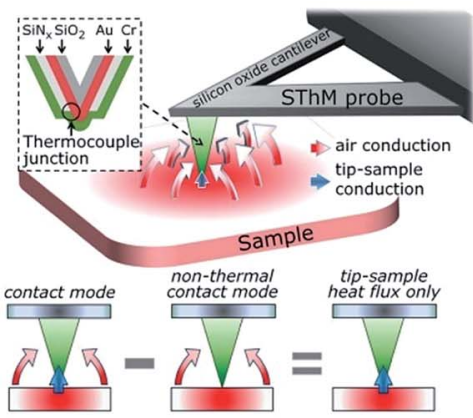

(d)

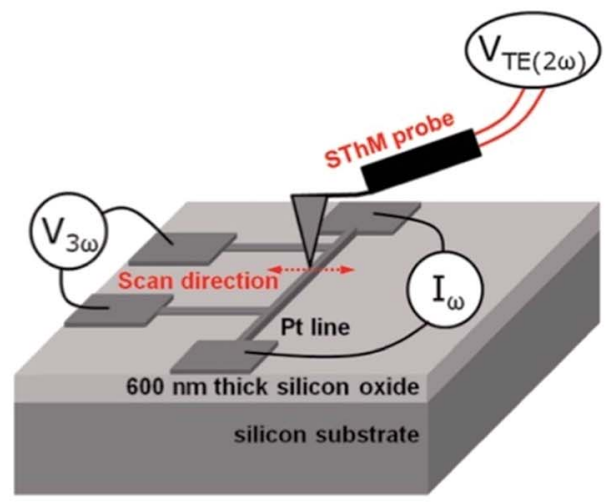

(f)

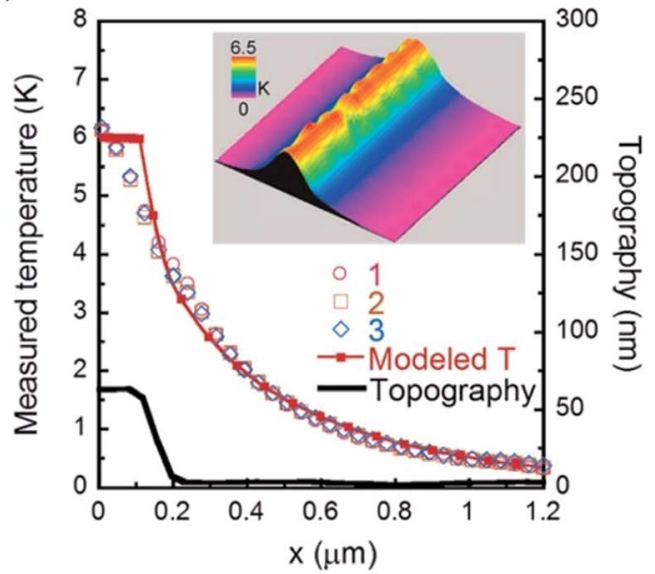

Fig. 19 (a) Experimental setup of SThM and (b) principle of quantitative thermal profiling. The SThM probe with a nano thermocouple junction integrated at the end of the tip, as shown in the inset, is mounted on an AFM. Below a scheme illustrating both contact mode and nonthermal contact mode is shown. (a, b) Reprinted with permission from ref. 390 Copyright (2011) American Chemical Society. (c) SEM micrographs of the SThM probe with an integrated AuCr thermocouple. The AuCr thermocouple junction is at the apex of the tip (d $\sim 100 \mathrm{~nm}$ ) (d) setup used to demonstrate quantitative thermal measurements. The temperature and topography of a heated Pt line are concomitantly mapped by scanning in a direction perpendicular to the Pt line. (e) Temperature profile obtained during slow scan, along with the modeled temperature profiles and the topographical profile of the Pt line. The inset depicts the contact conditions between the probe and the Pt line during the scan. (f) Temperature profile and topography obtained during fast scan. The inset depicts a four-dimensional map of the temperature field (color) and topography. The scan size was $200 \times 200 \mathrm{~nm}^{2}$. (c-f) Reprinted with permission from ref. 61 Copyright (2012) American Chemical Society.

Nevertheless, null-point SThM (NP-SThM) $)^{390}$ and the previously mentioned ultra-high vacuum method circumvent this limitation, and outstanding thermal $(\sim 15 \mathrm{mK})$ and spatial $(\sim 10 \mathrm{~nm})$ resolutions were obtained (see also Fig. 19d-f) ${ }^{61}$ Thermal conductivity is probed via the two omega $(2 \omega)$ method. ${ }^{387,388}$ To this end, an AC current at a frequency $\omega$ is applied to the thermoelectric probe. The current passing from the SThM probe to the sample surface via the thermocouple junction generates heat at a frequency of $2 \omega$, which leads to an oscillation of the junction temperature at $2 \omega$ and the amplitude of the $2 \omega$ signal from the thermocouple junction is monitored. ${ }^{387}$ Recently, a $2 \omega$ method was proposed utilizing 
a micro-thermocouple probe placed on a Quartz Tuning Fork (QTF), which circumvents the imprecise surface detection by optical deflection methods. ${ }^{391}$ Nevertheless, some artifacts persist during the measurement, for example Kim et al. observed a different temperature profile during the measurement of a heated Pt line in slow and fast scan mode, which was assigned to differences in contact area between tip and sample, resulting in a change in thermal resistance between the tip and the sample, as shown in Fig. 19e and f. ${ }^{61}$

(b) The change in electrical resistance can be utilized to determine the temperature at a small region on a sample. A heater with frequency $\omega$ heats the sample at $2 \omega$ frequency, similar to the $2 \omega$ method. The temperature oscillation at a frequency of $2 \omega$ results in an oscillation of the resistance of the second probe, which is thermal sensor, at $2 \omega$. (c) The fluorescence intensity depends strongly on the temperature, as the emitted intensity $I_{\mathrm{em}}$ is proportional to the population of excited states. By monitoring the fluorescence intensity at a given frequency, the temperature change can be derived from the change in fluorescence intensity. To enhance this approach, Aigouy et al. proposed gluing a fluorescent particle to the tip apex, which is afterward utilized for the local temperature measurement. ${ }^{378}$

(d) Thermal expansion (thermoacoustic effect) can be utilized to measure the increase of temperature as thermal expansion coefficient is usually in a range close to $10^{-5} \mathrm{~m} \mathrm{~K}^{-1}$ and the dilation can be measured accurately via AFM. The sample can be either heated by Joule heating ${ }^{392}$ or optically, ${ }^{393}$ similar to AFM-IR. ${ }^{289}$

Several approaches to enhance the lateral and thermal resolution have been investigated. In the beginning, in SThM (a)

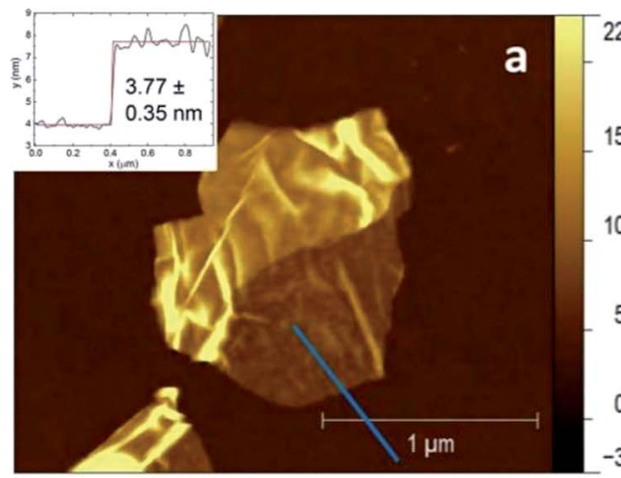

(b)

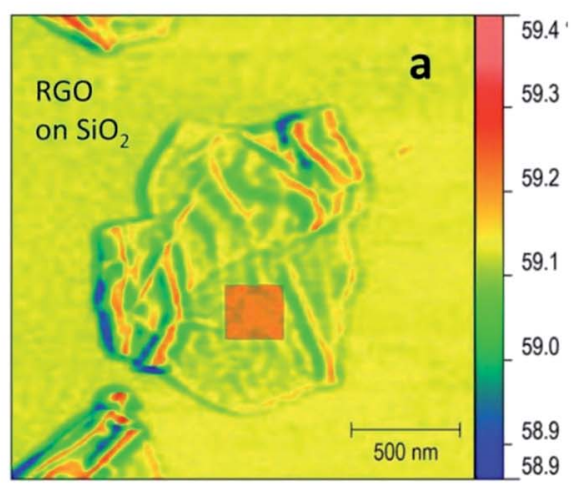

(c)

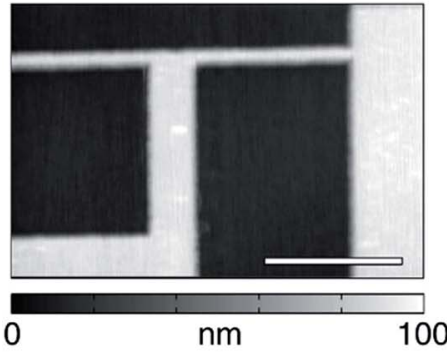

(d)

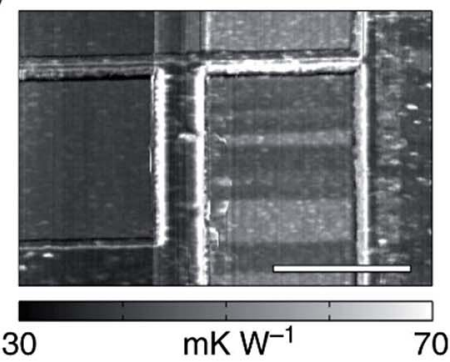

(e)

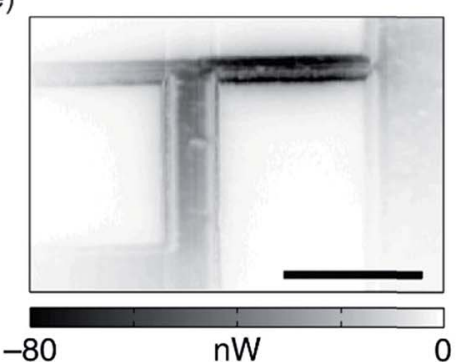

(f)

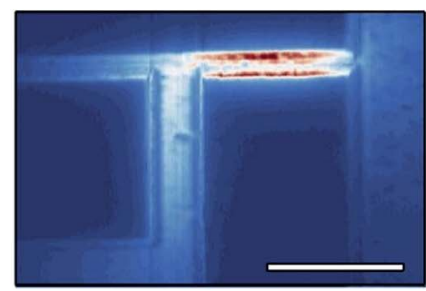

(g)

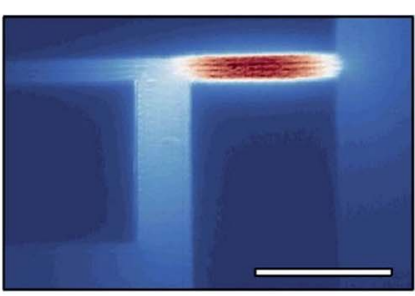

(h)

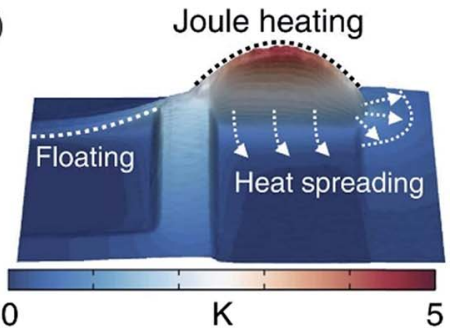

Fig. 20 Topography (a) and SThM (b) maps of RGO nanoplates deposited on a Si/SiO 2 . Reprinted with permission from ref. 405 Copyright (2016) Elsevier. A bipolar voltage bias is applied to the electrical interconnect shown in (c) as height image (scale bar, $1 \mathrm{~mm}$ ). Joule heating of the structure is characterized by simultaneously mapping local variations in (d) the tip-sample thermal resistance and (e) the tip-sample heat flux. ( $f$ ) Artefacts arising from constant, position-independent $(x, y)$ tip-sample thermal resistance (color bar see h) (g) calculated true sample temperature incline by combining position-dependent thermal resistance variations with the local heat flux maps. (h) Relative temperature increase encoded in both height and color, as well as heat spreading into the $\mathrm{SiN}_{\chi} / \mathrm{Si}$ substrate and the electrically floating interconnect segment. Reprinted from open-access article ref. 400 under the terms of the Creative Commons Attribution License. 
a Wollaston wire probe with a temperature coefficient $a=$ $0.00166 \mathrm{~K}^{-1}$ and time response of approximately $200 \mathrm{~ms}$ (in air) was utilized, which limits the lateral resolution of an experiment. ${ }^{394,395}$ Smaller metallic probes attached to cantilevers with a low spring constant $\left(0.35 \mathrm{~N} \mathrm{~m}^{-1}\right)$ and a tip radius of around $50 \mathrm{~nm}$ improved the lateral resolution. ${ }^{396,397}$ Furthermore, this probe enhanced the time response to a few tens of $\mathrm{ms}$ while maintaining a similar temperature coefficient as the Wollaston wire. ${ }^{398}$ Recently, high thermal conductivity nanowires (NW) attached to a tip apex were proposed to address the low efficiency of thermal coupling in SThM approach, while maintaining the lateral resolution. ${ }^{399}$ It has to be mentioned that the tip-sample heat transfer is crucial for the lateral resolution. Menges et al. developed a method, probing a timedependent and a time-averaged heat flux signal between a selfheated scanning probe sensor and a temperature-modulated sample, permitting the elimination of tip-sample contactrelated artifacts, a major step of SThM towards nanoscale thermometry. ${ }^{400}$

As stated previously, SThM can measure temperature (passive mode), thermal conductivity (active mode) and local phase-change transition (active mode) of a sample. The measurement of temperature is exploited for point studies and mappings of the amplitude of the steady periodic temperature field. It was utilized for the characterization of the temperature profile measurements of a PN thermoelectric couple ${ }^{401}$ and hot-spots in integrated circuits. ${ }^{402}$ Similar to KPFM, SThM in thermal mode appears useful for failure localization and analysis of integrated circuits. ${ }^{403,404}$ By the aforementioned smaller probes, heat dissipation, thermal conductivity or transport pathways in multiwall carbon nanotubes (MWCNs), single wall carbon nanotubes (SWCNs), graphene oxide (see Fig. 20a and b) ${ }^{405}$ and various other nanocomponents was determined. ${ }^{406}$ Furthermore, Joule selfheated nanomaterials, such as graphene nanoribbons (GNRs) ${ }^{407}$ silicon nanowire, nanowire diode ${ }^{408}$ and nanoscale metal interconnect structures (see Fig. $20 \mathrm{c}-\mathrm{h}$ ), ${ }^{400}$ and photoinduced heating ${ }^{409}$ were analyzed via this method. The thermal conductivity of materials was probed at the level of minuscule subsurface volumes for bulk $\mathrm{ZnO}$ and simple binary intermetallic clathrates $\left(\mathrm{Ba}_{8} \mathrm{Si}_{46}\right)$, porous bulks, ${ }^{410-413}$ mesoporous bulks ${ }^{414}$ and thin films of submicrometric thickness. ${ }^{415}$ Recently, SThM was utilized to determine the penetration depth of an adhesive in wood (subsurface imaging). ${ }^{47}$ Via the measurement of the heat conductivity, the phase transition between martensite-austenite of NiTi microstructures was accessible. ${ }^{416}$ Furthermore, the glass transition $T_{\mathrm{g}}$ and melting temperature $T_{\mathrm{m}}$ of polymers or polymer blends at submicron scale is accessible (method is also named nano-TA). ${ }^{368}$

\section{Conclusion and future aspects}

The combination of several orthogonal techniques is a fertile research field. Especially, hybrid analysis tools comprising out of two complementary techniques, which can compensate for their intrinsic weaknesses, are employed and refined while novel approaches are investigated in recent years. All these attempts to design hybrid analysis tools with nanometer resolution are driven by the search for advanced means to analyze and characterize new nanomaterials with high sensitivity and spatial resolution as well as to analyze processes occurring at the nanoscale, be it transport processes in biological samples or electronic processes at metal interfaces. Therefore, a strong emphasis was on combinations of optical methods, which are impeded by its low resolution, and AFM. Here, tremendous effort has been devoted to the improvement of resolution, signal-to-noise ratio and alignment/drift of the AFM. Furthermore, novel methods, such as SNEM and PTIR were actively developed, which yielded in the development of IR-PiFM, a noninvasive method of combined AFM and IR measurement with an unprecedentedly high optical resolution when compared with other IR measurement techniques. Still, some issues remain unsolved, for instance, the lifetime of the tip-apex in SNEM, PTIR and TERS is short, which results in a loss of signal after a few hours. Nonetheless, these tipenhanced methods are invaluable nowadays as they provide chemical fingerprints or chemical structures at sub-diffraction resolution. Combinations out of AFM and an optical microscopy can be advanced by adding further measurement techniques, such as fluorescence spectroscopy, fluorescence anisotropy and fluorescence lifetime. Although commercial solutions, at least for FLIM in combination with AFM are present, their alignment and computerized control still lack. These combined methods based on optical measurement techniques were mainly but not exclusively applied to biological samples (e.g. lipids, proteins, cells, viruses, particles, or biofilms), although these techniques also possess applications in material science and surface science (see surface nanobubbles).

In contrast, the non-optical techniques were predominantly utilized in applications related to material science and engineering (e.g. semiconductors and nanomaterials). Surface analysis techniques, relying on non-optical techniques, have emerged and effort has been devoted to these surface analysis techniques (KPFM and SThM) to improve their lateral resolution as well as their time resolution and precision. Still, resolution of these techniques is lower than for AFM on its own. Here, a variation of tip/tip-apex geometry and tip material has shown to enhance these parameters. Further advancement would benefit material science and engineering, as these methods are utilized to examine the properties of semiconductors for computers, which are presently undergoing miniaturization.

Some techniques, like QCM, SPR, FCS and RICM, are rarely combined with the AFM, which can be related to different acquisition times, issues with alignment and/or lack of data interpretation. However, these hybrid methods potentially give insight into processes appearing at interfaces at a molecular level. For example, despite the scarce application of RICM in literature, it has the potential to become a vital technique for determination of the actual contact area, which is important for adhesion measurements of soft colloidal probes. 
Table 1 Comprehensive list of hybrid methods based on AFM stated in this article

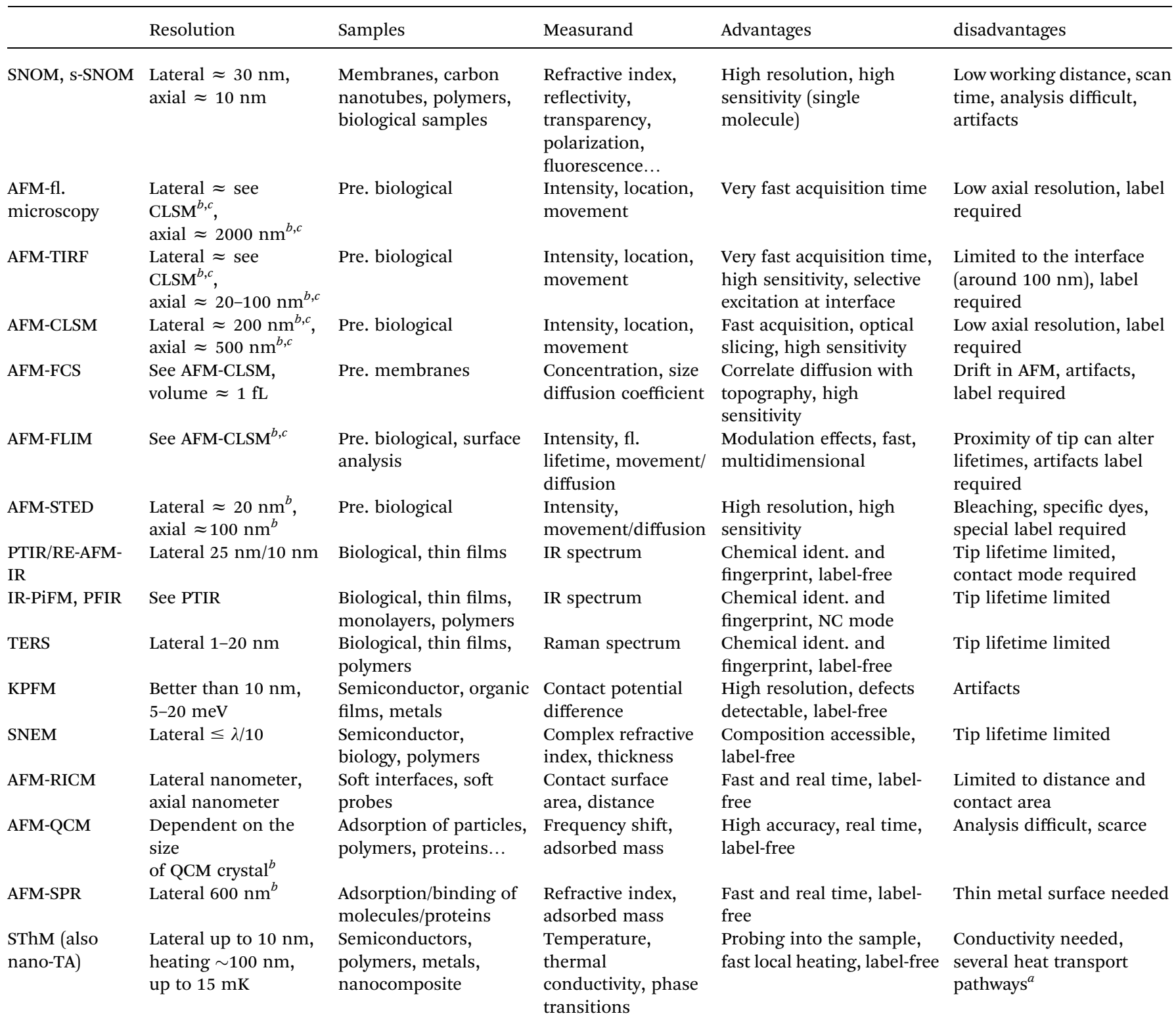

${ }^{a}$ For thermovoltage measurements. ${ }^{b}$ Resolution of the complementary technique, resolution of AFM remains sub nm (lateral) and sub Angström (axial). ${ }^{c}$ Tip enhancement effects may apply, pre. = predominant, fl. = fluorescence, ident. $=$ identification $\mathrm{NC}=$ noncontact.

In spite of the fact that most of the combined techniques are still under development and improvement, and equipment optimization is pending, hybrid techniques are highly important in the areas of biology, surface science (physical chemistry) and material science. Subsequently, Table 1 shows a comprehensive list of all major combined techniques mentioned in this review, together with their typical resolution, the field of application, advantages, and drawbacks, serving as a decision guidance for the interested reader. We anticipate that this review will provide a comprehensive overview of the hybrid measurement techniques based on the combination of surface sensitive techniques with the atomic force microscope. Besides, it will impart knowledge of the field of application of a specific combination in conjunction with its intrinsic advantages and limitations and thus serve as a viable tool for deciding on the most beneficial measurement method.

\section{Conflicts of interest}

There are no conflicts to declare.

\section{Acknowledgements}

We acknowledge the National Natural Science Foundation of China (21674064 and 21404072) for financial support of this work. We thank Lili Wang for fruitful discussions. 


\section{References}

1 P. Knittel, B. Mizaikoff and C. Kranz, Anal. Chem., 2016, 88, 6174-6178.

2 J. M. Mativetsky, Y.-L. Loo and P. Samori, J. Mater. Chem. C, 2014, 2, 3118-3128.

3 S. Liang, Y. Li, T. Zhou, J. Yang, X. Zhou, T. Zhu, J. Huang, J. Zhu, D. Zhu, Y. Liu, C. He, J. Zhang and X. Zhou, Adv. Sci., 2017, 4, 1600313.

4 I. S. Yahia, V. Ganesh, M. Shkir, S. AlFaify, H. Y. Zahran, H. Algarni, M. M. Abutalib, A. Al-Ghamdi, A. M. El-Naggar and A. M. AlBassam, Phys. B, 2016, 496, 9-14.

5 G. R. Luis and L. Jian, J. Phys.: Condens. Matter, 2009, 21, 483001.

6 M. D'Acunto, F. Dinelli and P. Pingue, Beilstein J. Nanotechnol., 2015, 6, 2278-2289.

7 J. A. Barreto, W. O'Malley, M. Kubeil, B. Graham, H. Stephan and L. Spiccia, Adv. Mater., 2011, 23, H18-H40.

8 Q. Huang, H. Wu, P. Cai, J. B. Fein and W. Chen, Sci. Rep., 2015, 5, 16857.

9 B. Song, K. Chen, M. Schmittel and H. Schönherr, Langmuir, 2016, 32, 11172-11178.

$10 \mathrm{H}$. Beyer, T. Wagner and A. Stemmer, Beilstein J. Nanotechnol., 2016, 7, 432-438.

11 B. Song, Y. Zhou and H. Schönherr, Langmuir, 2016, 32, 11179-11187.

12 C. Formosa and E. Dague, in Advanced Microscopy in Mycology, ed. S. T. E. Dahms and J. K. Czymmek, Springer International Publishing, Cham, 2015, pp. 125-141, DOI: 10.1007/978-3-319-22437-4_7.

13 M. Gaczynska, P. Karpowicz, C. E. Stuart, M. G. Norton, J. H. Teckman, E. Marszal and P. A. Osmulski, PLoS One, 2016, 11, 19.

14 R. Gahlin and S. Jacobson, Wear, 1998, 222, 93-102.

15 K. Miyahara, N. Nagashima, T. Ohmura and S. Matsuoka, Nanostruct. Mater., 1999, 12, 1049-1052.

16 S. Sundararajan and B. Bhushan, J. Mater. Res., 2001, 16, 437-445.

17 S. Amelio, A. V. Goldade, U. Rabe, V. Scherer, B. Bhushan and W. Arnold, Thin Solid Films, 2001, 392, 75-84.

18 I. A. Morozov, Macromolecules, 2016, 49, 5985-5992.

19 S. Z. Lian, R. Y. Shi, X. Huang, X. L. Hu, B. Song, Y. S. Bai, B. Yang, J. Y. Dong, Z. J. Du, Y. Y. Zhang, J. M. Jia, N. Ma, G. Guo and M. Y. Wang, Oncol. Rep., 2016, 36, 984-990.

20 Z. S. Fang, C. R. Jiang, Y. Feng, R. X. Chen, X. Y. Lin, Z. Q. Zhang, L. H. Han, X. D. Chen, H. Y. Li, Y. B. Guo and W. Y. Jiang, Biochim. Biophys. Acta, Mol. Cell Res., 2016, 1863, 2245-2254.

21 J. Li, C. Zhang, P. Cheng, X. Chen, W. Wang and J. Luo, Langmuir, 2016, 32, 5593-5599.

22 S. Senapati and S. Lindsay, Acc. Chem. Res., 2016, 49, 503510.

23 G. Zito, G. Rusciano, A. Vecchione, G. Pesce, R. Di Girolamo, A. Malafronte and A. Sasso, Sci. Rep., 2016, 6, 16.

24 X. L. Kou, W. Zhang and W. K. Zhang, ACS Appl. Mater. Interfaces, 2016, 8, 21055-21062.
25 X. Y. Hu, H. Z. Lei, X. Q. Zhang and Y. Zhang, Microsc. Res. Tech., 2016, 79, 721-726.

26 J. Onoda, K. Niki and Y. Sugimoto, Phys. Rev. B: Condens. Matter Mater. Phys., 2015, 92, 155309.

27 R. D. Whaley, B. Gopalan, M. Dagenais, R. D. Gomez, F. G. Johnson, S. Agarwala, O. King and D. R. Stone, J. Vac. Sci. Technol., B: Microelectron. Nanometer Struct.Process., Meas., Phenom., 1998, 16, 1007-1011.

28 B. Schuler, S. Fatayer, F. Mohn, N. Moll, N. Pavliček, G. Meyer, D. Peña and L. Gross, Nat. Chem., 2016, 8, 220224.

29 Q. Chen, H. Schonherr and G. J. Vancso, Soft Matter, 2009, 5, 4944-4950.

30 D. Hoffman, I. Miskioglu, K. E. Aifantis and J. Drelich, J. Adhes. Sci. Technol., 2012, 26, 1201-1220.

31 C. Kumari, K. Bhat and R. Bansal, J. Conservative Dent., 2016, 19, 56-62.

32 T. Hayashida and K. Umemura, Colloids Surf., B, 2016, 143, 526-531.

33 V. Horňáková, J. Přibyl and P. Skládal, Monatsh. Chem., 2016, 147, 865-871.

34 A. Wang, K. Vijayraghavan, O. Solgaard and M. J. Butte, ACS Nano, 2016, 10, 257-264.

35 M. Marchetti, G. J. L. Wuite and W. H. Roos, Curr. Opin. Virol., 2016, 18, 82-88.

36 E. Kämmer, I. Götz, T. Bocklitz, S. Stöckel, A. Dellith, D. Cialla-May, K. Weber, R. Zell, J. Dellith, V. Deckert and J. Popp, Anal. Bioanal. Chem., 2016, 408, 40354041.

37 C.-C. Wu, D. N. Reinhoudt, C. Otto, V. Subramaniam and A. H. Velders, Small, 2011, 7, 989-1002.

38 T. Gan, B. Wu, X. Zhou and G. Zhang, RSC Adv., 2016, 6, 50331-50335.

39 C. Chen, X. Zhou, Z. Xie, T. Gao and Z. Zheng, Small, 2015, 11, 613-621.

40 T. Gan, X. Zhou, C. Ma, X. Liu, Z. Xie, G. Zhang and Z. Zheng, Small, 2013, 9, 2850.

41 D. Alsteens, D. J. Müller and Y. F. Dufrêne, Acc. Chem. Res., 2017, 50, 924-931.

42 A. F. Eftaiha, S. M. K. Brunet and M. F. Paige, Current Microscopy Contributions to Advances in Science and Technology, Formatex, 2012, p. 1438.

43 D. H. Hu, M. Micic, N. Klymyshyn, Y. D. Suh and H. P. Lu, J. Lumin., 2004, 107, 4-12.

44 R. Kassies, K. O. V. d. Werf, A. Lenferink, C. N. Hunter, J. D. Olsen, V. Subramaniam and C. Otto, J. Microsc., 2005, 217, 109-116.

45 N. M. Sabanes, L. M. A. Driessen and K. F. Domke, Anal. Chem., 2016, 88, 7108-7114.

46 B. Lahiri, G. Holland and A. Centrone, Small, 2013, 9, 439445.

47 D. L. Xu, Y. Zhang, H. D. Zhou, Y. J. Meng and S. Q. Wang, Holzforschung, 2016, 70, 323-330.

48 L. Baldassarre, V. Giliberti, A. Rosa, M. Ortolani, A. Bonamore, P. Baiocco, K. Kjoller, P. Calvani and A. Nucara, Nanotechnology, 2016, 27, 075101. 
49 S. Ghosh, H. Remita, L. Ramos, A. Dazzi, A. DenisetBesseau, P. Beaunier, F. Goubard, P.-H. Aubert, F. Brisset and S. Remita, New J. Chem., 2014, 38, 1106-1115.

50 G. Hwang and O. Kwon, Nanoscale, 2016, 8, 5280-5290.

51 T. Tai, O. Karácsony, V. Bocharova, G. J. Van Berkel and V. Kertesz, Anal. Chem., 2016, 88, 2864-2870.

52 S. Moreno Flores and J. L. Toca-Herrera, Nanoscale, 2009, 1, 40-49.

53 J. M. Gerton, L. A. Wade, G. A. Lessard, Z. Ma and S. R. Quake, Phys. Rev. Lett., 2004, 93, 180801.

54 V. Walhorn, O. Schulz, C. Pelargus, D. Anselmetti and R. Ros, Proc. SPIE, 2007, 6444, 644406.

55 Y. Ebenstein, T. Mokari and U. Banin, J. Phys. Chem. B, 2004, 108, 93-99.

56 Z. Y. Ma, J. M. Gerton, L. A. Wade and S. R. Quake, Phys. Rev. Lett., 2006, 97, 260801-260804.

57 E. Yoskovitz, D. Oron, I. Shweky and U. Banin, J. Phys. Chem. C, 2008, 112, 16306-16311.

58 O. Schulz, Z. Zhao, A. Ward, M. König, F. Koberling, Y. Liu, J. Enderlein, H. Yan and R. Ros, Opt. Nanoscopy, 2013, 2, 1.

59 J. L. Garrett and J. N. Munday, Nanotechnology, 2016, 27, 245705.

60 E. Birkenhauer and S. Neethirajan, J. Visualized Exp., 2014, 93, e52327.

61 K. Kim, W. Jeong, W. Lee and P. Reddy, ACS Nano, 2012, 6, $4248-4257$.

62 S. Sadewasser, T. Glatzel, M. Rusu, A. Jäger-Waldau and M. C. Lux-Steiner, Appl. Phys. Lett., 2002, 80, 2979-2981.

63 P. Eaton and P. West, Atomic force microscopy, Oxford University Press Inc., New York, 2010.

64 H. Schönherr and G. J. Vancso, Scanning Force Microscopy of Polymers, Springer-Verlag, Berlin-Heidelberg, 2010.

$65 \mathrm{~J}$. Zhong and J. Yan, RSC Adv., 2016, 6, 1103-1121.

66 D. P. Allison, N. P. Mortensen, C. J. Sullivan and M. J. Doktycz, Wiley Interdiscip. Rev.: Nanomed. Nanobiotechnol., 2010, 2, 618-634.

67 Y. F. Dufrêne, T. Ando, R. Garcia, D. Alsteens, D. MartinezMartin, A. Engel, C. Gerber and D. J. Muller, Nat. Nanotechnol., 2017, 12, 295-307.

68 W. B. Amos and J. G. White, Biol. Cell, 2003, 95, 335-342.

69 A. Ostrowski, D. Nordmeyer, A. Boreham, C. Holzhausen, L. Mundhenk, C. Graf, M. C. Meinke, A. Vogt, S. Hadam, J. Lademann, E. Rühl, U. Alexiev and A. D. Gruber, Beilstein J. Nanotechnol., 2015, 6, 263-280.

70 M. Minsky, Microscopy Apparatus, US Pat., 3.013.467, 1957.

71 L. Schermelleh, R. Heintzmann and H. Leonhardt, J. Cell Biol., 2010, 190, 165-175.

72 K. D. Kihm, Near-Field Characterization of Micro/NanoScaled Fluid Flows, Springer, Berlin-Heidelberg, 2011.

73 M. Grossi, M. Morgunova, S. Cheung, D. Scholz, E. Conroy, M. Terrile, A. Panarella, J. C. Simpson, W. M. Gallagher and D. F. O'Shea, Nat. Commun., 2016, 7, 10855.

74 S. Liang, Y. Li, J. Yang, J. Zhang, C. He, Y. Liu and X. Zhou, Adv. Mater. Technol., 2016, 1, 1600117.

75 S. Liang, Y. Li, Y. Chen, J. Yang, T. Zhu, D. Zhu, C. He, Y. Liu, S. Handschuh-Wang and X. Zhou, J. Mater. Chem. C, 2017, 5, 1586-1590.
76 T. Zhou, J. Yang, D. Zhu, J. Zheng, S. Handschuh-Wang, X. Zhou, J. Zhang, Y. Liu, Z. Liu, C. He and X. Zhou, Adv. Sci., 2017, 4, 1700028.

77 D. Zhu, S. Handschuh-Wang and X. Zhou, J. Mater. Chem. A, 2017, 5, 16467-16497.

78 M. Bohrer, M. Schweitzer, R. Nirnberger and B. Weinberger, Proc. SPIE, 2015, 9636, 963609.

79 P. Ye, H. Yu and M. Houshmandi, BMC Oral Health, 2016, 16, 1-9.

80 S. Bosi, R. Rauti, J. Laishram, A. Turco, D. Lonardoni, T. Nieus, M. Prato, D. Scaini and L. Ballerini, Sci. Rep., 2015, 5, 9562.

81 M. Maglione and S. J. Sigrist, Nat. Neurosci., 2013, 16, 790797.

82 E. Abbe, Archiv für Mikroskopische Anatomie, 1873, 9, 413420.

83 S. Schnorrenberg, T. Grotjohann, G. Vorbruggen, A. Herzig, S. W. Hell and S. Jakobs, eLife, 2016, 5, e15567.

84 P. Hoyer, G. de Medeiros, B. Balazs, N. Norlin, C. Besir, J. Hanne, H. G. Krausslich, J. Engelhardt, S. J. Sahl, S. W. Hell and L. Hufnagel, Proc. Natl. Acad. Sci. U. S. A., 2016, 113, 3442-3446.

85 S. W. Hell, S. J. Sahl, M. Bates, X. W. Zhuang, R. Heintzmann, M. J. Booth, J. Bewersdorf, G. Shtengel, H. Hess, P. Tinnefeld, A. Honigmann, S. Jakobs, I. Testa, L. Cognet, B. Lounis, H. Ewers, S. J. Davis, C. Eggeling, D. Klenerman, K. I. Willig, G. Vicidomini, M. Castello, A. Diaspro and T. Cordes, J. Phys. D: Appl. Phys., 2015, 48, 443001.

86 S. W. Hell, Science, 2007, 316, 1153-1158.

87 S. W. Hell and J. Wichmann, Opt. Lett., 1994, 19, 780782 .

88 M. J. Broadhead, M. H. Horrocks, F. Zhu, L. Muresan, R. Benavides-Piccione, J. DeFelipe, D. Fricker, M. V. Kopanitsa, R. R. Duncan, D. Klenerman, N. H. Komiyama, S. F. Lee and S. G. N. Grant, Sci. Rep., 2016, 6, 24626.

89 A. Yildiz and P. R. Selvin, Acc. Chem. Res., 2005, 38, 574-582. 90 D. Kim, T. J. Deerinck, Y. M. Sigal, H. P. Babcock, M. H. Ellisman and X. Zhuang, PLoS One, 2015, 10, e0124581.

91 D. Wildanger, B. R. Patton, H. Schill, L. Marseglia, J. P. Hadden, S. Knauer, A. Schönle, J. G. Rarity, J. L. O'Brien, S. W. Hell and J. M. Smith, Adv. Mater., 2012, 24, 309-313.

92 C. Eggeling, K. I. Willig and F. J. Barrantes, J. Neurochem., 2013, 126, 203-212.

93 B. Harke, J. Keller and S. W. Hell, Opt. Express, 2008, 16, 4154-4162.

94 M. Dyba and S. W. Hell, Phys. Rev. Lett., 2002, 88, 163901. 95 J. Bückers, D. Wildanger, G. Vicidomini, L. Kastrup and S. W. Hell, Opt. Express, 2011, 19, 3130-3143.

96 E. Auksorius, B. R. Boruah, C. Dunsby, P. M. P. Lanigan, G. Kennedy, M. A. A. Neil and P. M. W. French, Opt. Lett., 2008, 33, 113-115.

97 M. Fernandez-Suarez and A. Y. Ting, Nat. Rev. Mol. Cell Biol., 2008, 9, 929-943. 
98 L. Po-Yen, L. Yi-Cheng, C. Chia-Seng and K. Fu-Jen, Jpn. J. Appl. Phys., 2013, 52, 028004.

99 C. S. Kim, X. Li, Y. Jiang, B. Yan, G. Y. Tonga, M. Ray, D. J. Solfiell and V. M. Rotello, MethodsX, 2015, 2, 306315.

100 K. M. Dean and A. E. Palmer, Nat. Chem. Biol., 2014, 10, 512-523.

101 A. Ettinger and T. Wittmann, in Methods in Cell Biology, ed. C. W. Jennifer and W. Torsten, Academic Press, 2014, vol. 123, pp. 77-94.

102 P. R. Sarika, N. R. James, P. R. A. Kumar and D. K. Raj, Mater. Sci. Eng., C, 2016, 68, 251-257.

103 N. Singh and S. C. Bhatla, Nitric Oxide Biol. Ch., 2016, 53, 54-64.

104 M. Wada, Plant Sci., 2013, 210, 177-182.

105 Y. C. Yao, H. Xu, C. Liu, Y. Y. Guan, D. Q. Xu, J. Y. Zhang, Y. L. Su, L. L. Zhao and J. B. Luo, RSC Adv., 2016, 6, 90829089.

106 M. J. Chen, C. M. Gao, S. Y. Lu, Y. M. Chen and M. Z. Liu, RSC Adv., 2016, 6, 9164-9174.

107 J. S. P. Wong, M. J. Hu, D. Shi, R. K. Y. Li and J. S. S. Wong, Polymer, 2015, 58, 67-75.

108 T. Wang, S. Handschuh-Wang, Y. Yang, H. Zhuang, C. Schlemper, D. Wesner, H. Schonherr, W. J. Zhang and X. Jiang, Langmuir, 2014, 30, 1089-1099.

109 N. Hain, D. Wesner, S. I. Druzhinin and H. Schönherr, Langmuir, 2016, 32, 11155-11163.

110 O. Schulz, F. Koberling, D. Walters, M. Koenig, J. Viani and R. Ros, Proc. SPIE, 2010, 7571, 757109.

111 C. Neagu, K. O. v. d. Werf, C. A. J. Putman, Y. M. Kraan, B. G. D. Grooth, N. F. v. Hulst and J. Greve, J. Struct. Biol., 1994, 112, 32-40.

112 K. Lieberman, N. Ben-Ami and A. Lewis, Rev. Sci. Instrum., 1996, 67, 3567.

113 M. d. Vestergaard, T. Hamada, M. Saito, Y. Yajima, M. Kudou, E. Tamiya and M. Takagi, Biochem. Biophys. Res. Commun., 2008, 377, 725-728.

114 S.-H. Jung, J.-Y. Park, J.-O. Yoo, I. Shin, Y.-M. Kim and K.-S. Ha, Ultramicroscopy, 2009, 109, 1428-1434.

115 S. Cazaux, A. Sadoun, M. Biarnes-Pelicot, M. Martinez, S. Obeid, P. Bongrand, L. Limozin and P.-H. Puech, Ultramicroscopy, 2016, 160, 168-181.

116 Y. R. Silberberg, A. E. Pelling, G. E. Yakubov, W. R. Crum, D. J. Hawkes and M. A. Horton, J. Mol. Recognit., 2008, 21, 30-36.

117 E. L. Grzywa, A. C. Lee, G. U. Lee and D. M. Suter, J. Neurobiol., 2006, 66, 1529-1543.

118 S. Kidoaki and T. Matsuda, J. Biomed. Mater. Res., Part A, 2007, 81, 803-810.

119 A. Sarkar, R. B. Robertson and J. M. Fernandez, Proc. Natl. Acad. Sci. U. S. A., 2004, 101, 12882-12886.

120 J. Gorelik, A. Shevchuk, M. Ramalho, M. Elliott, C. Lei, C. F. Higgins, M. J. Lab, D. Klenerman, N. Krauzewicz and Y. Korchev, Proc. Natl. Acad. Sci. U. S. A., 2002, 99, 16018-16023.

121 T. Timmel, M. Schuelke and S. Spuler, Microsc. Microanal., 2014, 20, 514-520.
122 A. Noy and T. R. Huser, Rev. Sci. Instrum., 2003, 74, 12171221.

123 R. Kassies, K. O. v. d. Werft, A. Lenferink, C. N. Hunter, J. D. Olsen, V. Subramaniam and C. Otto, J. Microsc., 2005, 217, 109-116.

124 J. R. Staunton, B. L. Doss, S. Lindsay and R. Ros, Sci. Rep., 2015, 6, 19686.

125 E. Gaviola, Z. Phys., 1925, 35, 748-756.

126 S. Cova, M. Bertolaccini and C. Bussolati, Phys. Status Solidi A, 1973, 18, 11-61.

127 S. Kinoshita and T. Kushida, Rev. Sci. Instrum., 1981, 52, $572-575$.

128 R. Schuyler and I. Isenberg, Rev. Sci. Instrum., 1971, 42, 813817.

129 T. Förster and G. Hoffmann, Z. Phys. Chem., 1971, 75, 6376.

130 M. Kaschke, J. Kleinschmidt and B. Wilhelmi, Laser Chem., 1985, 5, 119-132.

131 T. Nakabayashi, S. Oshita, R. Sumikawa, F. Sun, M. Kinjo and N. Ohta, J. Photochem. Photobiol., A, 2012, 235, 65-71.

132 V. Helenius, R. Monshouwer and R. v. Grondelle, J. Phys. Chem. B, 1997, 101, 10554-10559.

133 D. M. Togashi and A. G. Ryder, J. Fluoresc., 2006, 16, 153160.

134 M. Y. Berezin, H. Lee, W. Akers, G. Nikiforovich and S. Achilefu, Photochem. Photobiol. Sci., 2007, 83, 1371.

135 D. Magde, G. E. Rojas and P. G. Seybold, Photochem. Photobiol., 1999, 70, 737-744.

136 A. L. Koner, P. P. Mishra, S. Jha and A. Datta, J. Photochem. Photobiol., A, 2005, 170(1), 21-26.

137 D. A. Rusak, W. H. James, M. J. Ferzola and M. J. Stefanski, J. Chem. Educ., 2006, 83, 1857-1859.

138 S. Handschuh-Wang, T. Wang, S. I. Druzhinin, D. Wesner, X. Jiang and H. Schönherr, Langmuir, 2017, 33, 802-813.

139 S. Bernacchi and Y. Mély, Nucleic Acids Res., 2001, 29, e62.

140 D. Llères, S. Swift and A. I. Lamond, Curr. Protoc. Cytom., 2007, Chapter 12, Unit 12.10.

141 J. A. Lakowicz, H. Szmacinski, K. Nowaczyk and M. L. Johnson, Cell Calcium, 1992, 13, 131-147.

142 L. Seveus, M. Vaisala and E. Soini, Cytometry, 1992, 13, 329338.

143 S. Miersch, M. G. Espey, R. Chaube, A. Akarca, R. Tweten, S. Ananvoranich and B. Mutus, J. Biol. Chem., 2008, 283, 18513-18521.

144 S. Wang and Z.-R. Chen, Photochem. Photobiol. Sci., 2017, 16, 155-158.

145 S. Handschuh-Wang, D. Wesner, T. Wang, P. Lu, K.-S. Tücking, S. Haas, S. I. Druzhinin, X. Jiang and H. Schönherr, Macromol. Chem. Phys., 2017, 218, 1600454.

146 J. T. Krug, E. J. Sanchez and X. S. Xie, Appl. Phys. Lett., 2005, 86, 233102.

147 V. Protasenko, M. Kuno, A. Gallagher and D. Nesbitt, Opt. Commun., 2002, 210, 11-23.

148 J. R. T. Seddon, H. J. W. Zandvliet and D. Lohse, Phys. Rev. Lett., 2011, 107, 116101.

149 Y. He, V. Govind Rao, J. Cao and H. P. Lu, J. Phys. Chem. Lett., 2016, 7, 2221-2227. 
150 S. Patil, G. Matei, C. A. Grabowski, P. M. Hoffmann and A. Mukhopadhyay, Langmuir, 2007, 23, 4988-4992.

151 S. Chiantia, N. Kahya and P. Schwille, Langmuir, 2007, 23, 7659-7665.

152 A. R. Burns, D. J. Frankel and T. Buranda, Biophys. J., 2005, 89, 1081-1093.

153 S. Chiantia, N. Kahya, J. Ries and P. Schwille, Biophys. J., 2006, 90, 4500-4508.

154 S. Chiantia, J. Ries, G. Chwastek, D. Carrer, Z. Li, R. Bittman and P. Schwille, Biochim. Biophys. Acta, 2008, 1778, 13561364.

155 G. A. Rojas, Y. Wu, G. Haugstad and C. D. Frisbie, ACS Appl. Mater. Interfaces, 2016, 8, 5772-5776.

156 M. S. Itano, A. K. Neumann, P. Liu, F. Zhang, E. Gratton, W. J. Parak, N. L. Thompson and K. Jacobson, Biophys. J., 2011, 100, 2662-2670.

157 J. Kuchlyan, N. Kundu, D. Banik, A. Roy and N. Sarkar, Langmuir, 2015, 31, 13793-13801.

158 X. W. Ng, C. Teh, V. Korzh and T. Wohland, Biophys. J., 2016, 111, 418-429.

159 J. Widengren, U. Mets and R. Rigler, J. Phys. Chem. B, 1995, 99, 13368-13379.

160 N. Biswas, R. Bhattacharya, A. Saha, N. R. Janab and J. K. Basu, Phys. Chem. Chem. Phys., 2015, 17, 24238-24247.

161 S. Chiantia, J. Ries, N. Kahya and P. Schwille, ChemPhysChem, 2006, 7, 2409.

162 D. R. Stabley, T. Oh, S. M. Simon, A. L. Mattheyses and K. Salaita, Nat. Commun., 2015, 6, 8307.

163 Y. Fu, P. W. Winter, R. Rojas, V. Wang, M. McAuliffe and G. H. Patterson, Proc. Natl. Acad. Sci. U. S. A., 2016, 113, 4368-4373.

164 C. U. Chan and C.-D. Ohl, Phys. Rev. Lett., 2012, 109, 174501.

165 J. Ki, P. Arumugam and J. M. Song, Anal. Bioanal. Chem., 2016, 408, 3233-3238.

166 S. Block, V. P. Zhdanov and F. Höök, Nano Lett., 2016, 16, 4382-4390.

167 T. Yamada, R. Afrin, H. Arakawa and A. Ikai, FEBS Lett., 2004, 569, 59-64.

168 A. E. X. Brown, A. Hategan, D. Safer, Y. E. Goldman and D. E. Discher, Biophys. J., 2009, 96, 1952-1960.

169 M. S. Z. Kellermayer, A. Karsai, A. Kengyel, A. Nagy, P. Bianco, T. Huber, A. Kulcsar, C. Niedetzky, R. Proksch and L. Grama, Biophys. J., 2006, 91, 2665-2677.

170 R. Eckel, V. Walhorn, C. Pelargus, J. Martini, J. Enderlein, T. Nann, D. Anselmetti and R. Ros, Small, 2007, 3, 44-49.

171 A. Choucair, M. Chakrapani, B. Chakravarthy, L. Katsaras and L. J. Johnston, Biochim. Biophys. Acta, 2007, 1786, 146-154.

172 W. Christenson, I. Yermolenko, B. Plochberger, F. Camacho-Alanis, A. Ros, T. P. Ugarova and R. Ros, Ultramicroscopy, 2014, 136, 211-215.

173 A. B. Mathur, G. A. Truskey and W. M. Reichert, Biophys. J., 2000, 78, 1725-1735.

174 A. Ortega-Esteban, K. Bodensiek, C. San Martín, M. Suomalainen, U. F. Greber, P. J. de Pablo and I. A. T. Schaap, ACS Nano, 2015, 9, 10571-10579.
175 S. Nishida, Y. Funabashi and A. Ikai, Ultramicroscopy, 2002, 91, 269-274.

176 P. Anger, P. Bharadwadj and L. Novotny, Phys. Rev. Lett., 2006, 96, 113002.

177 S. Y. Lee, K. Nakaya, T. Hayashiab and M. Hara, Phys. Chem. Chem. Phys., 2009, 11, 4403-4409.

178 V. Walhorn, J. Paskarbeit, H. G. Frey, A. Harder and D. Anselmetti, Beilstein J. Nanotechnol., 2011, 2, 645-652.

179 D. Milovanovic, M. Platen, M. Junius, U. Diederichsen, I. A. T. Schaap, A. Honigmann, R. Jahn and G. van den Bogaart, J. Biol. Chem., 2016, 291, 7868-7876.

180 M. Capitanio, D. Maggi, F. Vanzi and F. S. Pavone, J. Opt. A: Pure Appl. Opt., 2007, 9, S157-S163.

181 P. D. Odermatt, A. Shivanandan, H. Deschout, R. Jankele, A. P. Nievergelt, L. Feletti, M. W. Davidson, A. Radenovic and G. E. Fantner, Nano Lett., 2015, 15, 4896-4904.

182 D. Wustner, M. Modzel, F. W. Lund and M. A. Lomholt, Chem. Phys. Lipids, 2016, 199, 106-135.

183 M. Koenig, P. Reisch, R. Dowler, B. Kraemer, S. Tannert, M. Patting, M. P. Clausen, S. Galiani, C. Eggeling, F. Koberling and R. Erdmann, Proc. SPIE, 2016, 9712, 97120T.

184 I. Testa, E. D'Este, N. T. Urban, F. Balzarotti and S. W. Hell, Nano Lett., 2015, 15, 103-106.

185 J. Tonnesen, G. Katona, B. Rozsa and U. V. Nagerl, Nat. Neurosci., 2014, 17, 678-685.

186 A. N. Butkevich, G. Y. Mitronova, S. C. Sidenstein, J. L. Klocke, D. Kamin, D. N. H. Meineke, E. D'Este, P. T. Kraemer, J. G. Danzl, V. N. Belov and S. W. Hell, Angew. Chem., Int. Ed., 2016, 55, 3290-3294.

187 S. C. Sidenstein, E. D'Este, M. J. Bohm, J. G. Danzl, V. N. Belov and S. W. Hell, Sci. Rep., 2016, 6, 26725.

188 N. Kodera, D. Yamamoto, R. Ishikawa and T. Ando, Nature, 2010, 468, 72-76.

189 J. C. Cordova, D. K. Das, H. W. Manning and M. J. Lang, Curr. Opin. Struct. Biol., 2014, 28, 142-148.

190 J. V. Chacko, B. Harke, C. Canale and A. Diaspro, J. Biomed. Opt., 2014, 19, 105003.

191 B. Harke, J. V. Chacko, H. Haschke, C. Canale and A. Diaspro, Opt. Nanoscopy, 2012, 1, 3.

192 T. Deguchi, S. Koho, T. Nareoja and P. Hanninen, Opt. Rev., 2014, 21, 389-394.

193 J. V. Chacko, C. Canale, B. Harke and A. Diaspro, PLoS One, 2013, 8, e66608.

194 J. Q. Yu, J. H. Yuan, X. J. Zhang, J. L. Liu and X. H. Fang, Chin. Sci. Bull., 2013, 58, 4045-4050.

195 D. N. Fronczek, C. Quammen, H. Wang, C. Kisker, R. Superfine, R. Taylor, D. A. Erie and I. Tessmer, Ultramicroscopy, 2011, 111, 350-355.

196 E. A. Ash and O. Nichols, Nature, 1972, 237, 510-512.

197 Y. Oshikane, T. Kataoka, M. Okuda, S. Hara, H. Inoue and M. Nakano, Sci. Technol. Adv. Mater., 2007, 8, 181-185.

198 F. Keilmann and R. Hillenbrand, Phil. Trans. R. Soc. A, 2004, 362, 787-805.

199 T. J. Yang, G. A. Lessard and S. R. Quake, Appl. Phys. Lett., 2000, 76, 378-380. 
200 F. Zenhausern, M. P. O'Boyle and H. K. Wickramasinghe, Appl. Phys. Lett., 1994, 65, 1623.

201 Y. Martin, F. Zenhausern and H. K. Wickramasinghe, Appl. Phys. Lett., 1996, 68, 2475.

202 A. Kramer, W. Trabesinger, B. Hecht and U. P. Wild, Appl. Phys. Lett., 2002, 80, 1652.

203 N. Hayazawa, Y. Inouye and S. Kawata, J. Microsc., 1999, 194, 472-476.

204 J. Azoulay, A. Débarre, A. Richard and P. Tchénio, J. Microsc., 1999, 194, 486.

205 A. Hartschuh, E. J. Sánchez, X. S. Xie and L. Novotny, Phys. Rev. Lett., 2003, 90, 095503.

206 T. Ichimura, N. Hayazawa, M. Hashimoto, Y. Inouye and S. Kawata, Phys. Rev. Lett., 2004, 92, 220801.

207 H. G. Frey, J. Paskarbeit and D. Anselmetti, Appl. Phys. Lett., 2009, 94, 241116.

208 N. Behr and M. Raschke, J. Phys. Chem. C, 2008, 112, 37663773.

209 W.-S. Chang, S. Bauerdick and M. S. Jeong, Ultramicroscopy, 2008, 108, 1070-1075.

210 Z. Shi, X. Hong, H. A. Bechtel, B. Zeng, M. C. Martin, K. Watanabe, T. Taniguchi, Y.-R. Shen and F. Wang, Nat. Photonics, 2015, 9, 515-519.

211 B. Pollard, E. A. Muller, K. Hinrichs and M. B. Raschke, Nat. Commun., 2014, 5, 3587.

212 S. A. Donges, O. Khatib, B. T. O'Callahan, J. M. Atkin, J. H. Park, D. Cobden and M. B. Raschke, Nano Lett., 2016, 16, 3029-3035.

213 N. El-Kork, P. Moretti, B. Jacquier, F. Lei and M. Ismail, J. Nanomater., 2016, 2016, 4089260.

214 L. Andolfi, E. Trevisan, B. Troian, S. Prato, R. Boscolo, E. Giolo, S. Luppi, M. Martinelli, G. Ricci and M. Zweyer, J. Nanobiotechnol., 2015, 13, 2.

215 Y. De Wilde and P. A. Lemoine, AIP Conf. Proc., 2007, 931, 43-52.

216 R. Gaifulina, A. T. Maher, C. Kendall, J. Nelson, M. Rodriguez-Justo, K. Lau and G. M. Thomas, Int. J. Exp. Pathol., 2016, 97, 337-350.

217 A. K. Meher and Y.-C. Chen, Anal. Chem., 2016, 88, 91519157.

218 K. Luo, Y. D. Xiang, H. M. Wang, L. Xiang and Z. H. Luo, J. Mater. Sci. Technol., 2016, 32, 733-737.

219 C. S. Casari, C. S. Giannuzzi and V. Russo, Carbon, 2016, 104, 190-195.

220 T. Kavinkumar and S. Manivannan, J. Mater. Sci. Technol., 2016, 32, 626-632.

221 W. Miranda, S. S. Coutinho, M. S. Tavares, E. Moreira and D. L. Azevedo, J. Mol. Struct., 2016, 1122, 299-308.

222 P. K. Chu and L. Li, Mater. Chem. Phys., 2006, 96, 253277.

223 E. V. Efremov, F. Ariese and C. Gooijer, Anal. Chim. Acta, 2008, 606, 119-134.

224 S. Bonhommeau, D. Talaga, J. Hunel, C. Cullin and S. Lecomte, Angew. Chem., 2017, 129, 1797-1800.

225 P. C. Gutiérrez-Neira, F. Agulló-Rueda, A. Climent-Font and C. Garrido, Vib. Spectrosc., 2013, 69, 13-20.
226 S. Boyd, M. F. Bertino and S. J. Seashols, Forensic Sci. Int., 2011, 208, 124-128.

227 G. Barone, D. Bersani, J. Jehlicka, P. P. Lottici, P. Mazzoleni, S. Raneri, P. Vandenabeele, C. Di Giacomo and G. Larina, J. Raman Spectrosc., 2015, 46, 989-995.

228 M. N. Kinalwa, E. W. Blanch and A. J. Doig, Anal. Chem., 2010, 82, 6347-6349.

229 S. Signorelli, S. Cannistraro and A. R. Bizzarri, Appl. Spectrosc., 2016, 71, 823-832.

230 H. J. Butler, L. Ashton, B. Bird, G. Cinque, K. Curtis, J. Dorney, K. Esmonde-White, N. J. Fullwood, B. Gardner, P. L. Martin-Hirsch, M. J. Walsh, M. R. McAinsh, N. Stone and F. L. Martin, Nat. Protoc., 2016, 11, 664-687.

231 N. Kumar, M. M. Drozdz, H. Jiang, D. M. Santos and D. J. Vaux, Chem. Commun., 2017, 53, 2451-2454.

232 P. Mukherjee, S. J. Lim, T. P. Wrobel, R. Bhargava and A. M. Smith, J. Am. Chem. Soc., 2016, 138, 10887-10896.

233 T. Iwasaki, T. Zelai, S. Ye, Y. Tsuchiya, H. M. H. Chong and H. Mizuta, Carbon, 2017, 111, 67-73.

234 A. Paudel, D. Raijada and J. Rantanen, Adv. Drug Delivery Rev., 2015, 89, 3-20.

235 L. S. Lawson and J. D. Rodriguez, Anal. Chem., 2016, 88, 4706-4713.

236 M. Asghari-Khiavi, B. R. Wood, P. Hojati-Talemi, A. Downes, D. McNaughtona and A. Mechlera, J. Raman Spectrosc., 2012, 43, 173-180.

237 W. Su and D. Roy, J. Vac. Sci. Technol., B: Nanotechnol. Microelectron.: Mater., Process., Meas., Phenom., 2013, 31, 041808.

238 N. Kumar, B. Stephanidis, R. Zenobi, A. J. Wain and D. Roy, Nanoscale, 2015, 7, 7133-7137.

239 R. Zhang, Y. Zhang, Z. C. Dong, S. Jiang, C. Zhang and L. G. Chen, Nature, 2013, 498, 82-86.

240 N. Kumar, S. Mignuzzi, W. Su and D. Roy, EPJ Tech. Instrum., 2015, 2, 9.

241 B. R. Wood, E. Bailo, M. A. Khiavi, L. Tilley, S. Deed and T. Deckert-Gaudig, Nano Lett., 2011, 11, 1868-1873.

242 R. Bohme, M. Richter, D. Cialla, P. Rosch, V. Deckert and J. Popp, J. Raman Spectrosc., 2009, 40, 1452-1457.

243 T. Deckert-Gaudig, E. Bailo and V. Deckert, Phys. Chem. Chem. Phys., 2009, 11, 7360-7362.

244 L. M. Malard, M. A. Pimenta, G. Dresselhaus and M. S. Dresselhaus, Phys. Rep., 2009, 473, 51-87.

245 E. M. v. S. Lantman, T. Deckert-Gaudig, A. J. G. Mank, V. Deckert and B. M. Weckhuysen, Nat. Nanotechnol., 2012, 7, 583-586.

246 C. E. Harvey and B. M. Weckhuysen, Catal. Lett., 2015, 145, 40-57.

247 T. Hartman, C. S. Wondergem, N. Kumar, A. v. d. Berg and B. M. Weckhuysen, J. Phys. Chem. Lett., 2016, 7, 1570-1584.

248 N. Jiang, D. Kurouski, E. A. Pozzi, N. Chiang, M. C. Hersam and R. P. V. Duyne, Chem. Phys. Lett., 2016, 659, 16-24.

249 T. Mino, Y. Saito and P. Verma, ACS Nano, 2014, 8, 1018710195.

250 L. T. Nieman, G. M. Krampert and R. E. Martinez, Rev. Sci. Instrum., 2001, 72, 1691-1699. 
251 L. Opilik, Ü. Dogan, J. Szczerbinski and R. Zenobi, Appl. Phys. Lett., 2015, 107, 091109.

252 Y. Fujita, R. Chiba, G. Lu, N. N. Horimoto, S. Kajimoto, H. Fukumura and H. Uji-i, Chem. Commun., 2014, 50, 9839-9841.

253 C. A. Barrios, A. V. Malkovskiy, A. M. Kisliuk, A. P. Sokolov and M. D. Foster, J. Phys. Chem. C, 2009, 113, 8158-8161.

254 N. Kumar, S. J. Spencer, D. Imbraguglio, A. M. Rossi, A. J. Wain, B. M. Weckhuysen and D. Roy, Phys. Chem. Chem. Phys., 2016, 18, 13710-13716.

255 P. Pienpinijtham, S. Vantasin, Y. Kitahama, S. Ekgasit and Y. Ozaki, J. Phys. Chem. C, 2016, 120, 14663-14668.

256 N. Biliškov and G. Baranović, J. Mol. Liq., 2009, 144, 155162.

257 S. Nakata, M.-a. Kakimoto and Y. Imai, Polym. J., 1993, 25, 569-576.

258 A. Harrer, R. Szedlak, B. Schwarz, H. Moser, T. Zederbauer, D. MacFarland, H. Detz, A. M. Andrews, W. Schrenk, B. Lendl and G. Strasser, Sci. Rep., 2016, 6, 21795.

259 M. F. H. Al-Kadhemy, Z. S. Rasheed and S. R. Salim, J. Radiat. Res. Appl. Sci., 2016, 9, 321-331.

260 S. Prati, E. Joseph, G. Sciutto and R. Mazzeo, Acc. Chem. Res., 2010, 43, 792-801.

261 R. Bhargava and I. W. Levin, in Spectrochemical Analysis Using Infrared Multichannel Detectors, Blackwell Publishing Ltd, 2007, pp. 1-24, DOI: 10.1002/ 9780470988541.ch1.

262 M. M. Beasley, E. J. Bartelink, L. Taylor and R. M. Miller, J. Archaeol. Sci., 2014, 46, 16-22.

263 J. S. Gaffney, N. A. Marley and D. E. Jones, in Characterization of Materials, John Wiley \& Sons, Inc., 2002, DOI: 10.1002/0471266965.com107.pub2.

264 Á. I. López-Lorente and B. Mizaikoff, Anal. Bioanal. Chem., 2016, 408, 2875-2889.

265 N. Brandes, P. B. Welzel, C. Werner and L. W. Kroh, J. Colloid Interface Sci., 2006, 299, 56-69.

266 A. E. Guber and U. Köhler, J. Mol. Struct., 1995, 348, 209212.

267 M. B. Esler, D. W. T. Griffith, S. R. Wilson and L. P. Steele, Anal. Chem., 2000, 72, 206-215.

268 R. C. Pereira, M. C. Arbestain, M. V. Sueiro and J. A. MaciáAgulló, Soil Res., 2015, 53, 753-762.

269 K. Kachel, D. Siche, S. Golka, P. Sennikov and M. Bickermann, Mater. Chem. Phys., 2016, 177, 12-18.

270 O. Planinšek, D. Planinšek, A. Zega, M. Breznik and S. Srčič, Int. J. Pharm., 2006, 319, 13-19.

271 A. Francis, R. Detsch and A. R. Boccaccini, Ceram. Int., 2016, 42, 15442-15448.

272 W. H. Zheng, J. T. Hu, S. Rappeport, Z. Zheng, Z. X. Wang, Z. S. Han, J. Langer and J. Economy, Microporous Mesoporous Mater., 2016, 234, 146-154.

273 T. Wang, S. Handschuh-Wang, P. Qin, Y. Yang, X. Zhou and Y. Tang, J. Colloid Interface Sci., 2017, 499, 102-109.

274 T. Wang, S. Handschuh-Wang, S. Zhang, X. Zhou and Y. Tang, J. Colloid Interface Sci., 2017, 506, 543-552.

275 Y. Kano, K. Ishikura, S. Kawahara and S. Akiyama, Polym. J., 1992, 24, 135-144.
276 Y. Fazli, H. Alijani and K. Khezri, Adv. Polym. Technol., 2016, 35, 260-268.

277 A. K. Pal and V. Katiyar, Biomacromolecules, 2016, 17, 26032618.

278 A. Dazzi, F. Glotin and R. Carminati, J. Appl. Phys., 2010, 107, 124519.

279 L. Baldassarre, V. Giliberti, A. Rosa, M. Ortolani, A. Bonamore, P. Baiocco, K. Kjoller, P. Calvani and A. Nucara, Nanotechnology, 2016, 27, 075101.

280 T. Muller, F. S. Ruggeri, A. J. Kulik, U. Shimanovich, T. O. Mason, T. P. J. Knowles and G. Dietler, Lab Chip, 2014, 14, 1315-1319.

281 P. Vitry, R. Rebois, E. Bourillot, A. Deniset-Besseau, M.-J. Virolle, E. Lesniewska and A. Dazzi, Nano Res., 2016, 9, 1674-1681.

282 C. Marcott, M. Lo, K. Kjoller, F. Fiat, N. Baghdadli, G. Balooch and G. S. Luengo, Appl. Spectrosc., 2014, 68, 564-569.

283 C. Policar, J. B. Waern, M.-A. Plamont, S. Clède, C. Mayet, R. Prazeres, J.-M. Ortega, A. Vessières and A. Dazzi, Angew. Chem., Int. Ed., 2011, 50, 860-864.

284 E. Kennedy, R. Al-Majmaie, M. Al-Rubeai, D. Zerulla and J. H. Rice, RSC Adv., 2013, 3, 13789-13795.

285 A. Deniset-Besseau, C. B. Prater, M.-J. Virolle and A. Dazzi, J. Phys. Chem. Lett., 2014, 5, 654-658.

286 F. G. Tang, P. T. Bao and Z. H. Su, Anal. Chem., 2016, 88, 4926-4930.

287 M. Kelchtermans, M. Lo, E. Dillon, K. Kjoller and C. Marcott, Vib. Spectrosc., 2016, 82, 10-15.

288 A. M. Katzenmeyer, V. Aksyuk and A. Centrone, Anal. Chem., 2013, 85, 1972-1979.

289 A. Dazzi, J. Saunier, K. Kjoller and N. Yagoubi, Int. J. Pharm., 2015, 484, 109-114.

290 B. Van Eerdenbrugh, M. Lo, K. Kjoller, C. Marcott and L. S. Taylor, J. Pharm. Sci., 2012, 101, 2066-2073.

291 A. J. Harrison, E. A. Bilgili, S. P. Beaudoin and L. S. Taylor, Anal. Chem., 2013, 85, 11449-11455.

292 S. Morsch, Y. W. Liu, S. B. Lyon and S. R. Gibbon, ACS Appl. Mater. Interfaces, 2016, 8, 959-966.

293 L. Gong, D. B. Chase, I. Noda, J. L. Liu, D. C. Martin, C. Y. Ni and J. F. Rabolt, Macromolecules, 2015, 48, 6197-6205.

294 V. Aksyuk, B. Lahiri, G. Holland and A. Centrone, Nanoscale, 2015, 7, 3634-3644.

295 S. Laokroekkiat, M. Hara, S. Nagano and Y. Nagao, Langmuir, 2016, 32, 6648-6655.

296 J. Chae, Q. F. Dong, J. S. Huang and A. Centrone, Nano Lett., 2015, 15, 8114-8121.

297 Y. B. Yuan, J. Chae, Y. C. Shao, Q. Wang, Z. G. Xiao, A. Centrone and J. S. Huang, Adv. Energy Mater., 2015, 5, 1500615.

298 M. K. F. Lo, A. Dazzi, C. A. Marcott, E. Dillon, Q. C. Hu, K. Kjoller, C. B. Prater and S. W. King, ECS J. Solid State Sci. Technol., 2016, 5, P3018-P3024.

299 F. Lu, M. Z. Jin and M. A. Belkin, Nat. Photonics, 2014, 8, 307-312.

300 F. Lu and M. A. Belkin, Opt. Express, 2011, 19, 1994219947. 
301 P. Vitry, E. Bourillot, C. Plassard, Y. Lacroute, E. Calkins, L. Tetard and E. Lesniewska, Nano Res., 2015, 8, 2199-2205. 302 L. Wang, H. Wang, M. Wagner, Y. Yan, D. S. Jakob and X. G. Xu, Sci. Adv., 2017, 3, e1700255.

303 A. Ambrosio, R. C. Devlin, F. Capasso and W. L. Wilson, ACS Photonics, 2017, 4, 846-851.

304 D. Nowak, W. Morrison, H. K. Wickramasinghe, J. Jahng, E. Potma, L. Wan, R. Ruiz, T. R. Albrecht, K. Schmidt, J. Frommer, D. P. Sanders and S. Park, Sci. Adv., 2016, 2, e1501571.

305 J. Jahng, D. A. Fishman, S. Park, D. B. Nowak, W. A. Morrison, H. K. Wickramasinghe and E. O. Potma, Acc. Chem. Res., 2015, 48, 2671-2679.

306 J. N. Hilfiker, M. Stadermann, J. Sun, T. Tiwald, J. S. Hale, P. E. Miller and C. Aracne-Ruddle, Appl. Surf. Sci., 2017, 421, 508-512.

307 S. A. Kukushkin and A. V. Osipov, Tech. Phys. Lett., 2016, 42, 175-178.

308 J. J. Diaz Leon, D. M. Fryauf, R. D. Cormia, M.-X. M. Zhang, K. Samuels, R. S. Williams and N. P. Kobayashi, ACS Appl. Mater. Interfaces, 2016, 8, 22337-22344.

309 S. Wang, J. Rond, C. Steinberg, M. Papenheim and H.-C. Scheer, Appl. Phys. A: Mater. Sci. Process., 2016, 122, 121.

310 K. Dorywalski, I. Maciejewski and T. Krzyżyński, Mechatronics, 2016, 37, 33-41.

311 C.-H. Zhai, R.-J. Zhang, X. Chen, Y.-X. Zheng, S.-Y. Wang, J. Liu, N. Dai and L.-Y. Chen, Nanoscale Res. Lett., 2016, 11, 407.

312 P. Karageorgiev, H. Orendi, B. Stiller and L. Brehmer, Appl. Phys. Lett., 2001, 79, 1730-1732.

313 D. Tranchida, J. Diaz, P. Schon, H. Schonherr and G. J. Vancso, Nanoscale, 2011, 3, 233-239.

314 A. Cumurcu, J. Diaz, I. D. Lindsay, S. de Beer, J. Duvigneau, P. Schon and G. J. Vancso, Ultramicroscopy, 2015, 150, 7987.

315 A. Cumurcu, J. Duvigneau, I. D. Lindsay, P. M. Schon and G. J. Vancso, Eur. Polym. J., 2013, 49, 1935-1942.

316 A. S. G. Curtis, J. Cell Biol., 1964, 20, 199-215.

317 L. Limozin and K. Sengupta, ChemPhysChem, 2009, 10, 2752-2768.

318 J. Erath, S. Schmidt and A. Fery, Soft Matter, 2010, 6, 14321437.

319 D. Gingell and I. Todd, Biophys. J., 1979, 26, 507-526.

320 S. Attili and R. P. Richter, Langmuir, 2012, 28, 32063216.

321 S. Schmidt, H. Wang, D. Pussak, S. Mosca and L. Hartmann, Beilstein J. Org. Chem., 2015, 11, 720-729.

322 E. Wassel, S. Jiang, Q. Song, S. Vogt, G. Nöll, S. I. Druzhinin and H. Schönherr, Langmuir, 2016, 32, 9360-9370.

323 P. Palladino, A. M. Aura and G. Spoto, Anal. Bioanal. Chem., 2016, 408, 849-854.

324 K. Johansen, H. Arwin, I. Lundström and B. Liedberg, Rev. Sci. Instrum., 2000, 71, 3530-3538.

325 A. Zuber, M. Purdey, E. Schartner, C. Forbes, B. van der Hoek, D. Giles, A. Abell, T. Monro and H. EbendorffHeidepriem, Sens. Actuators, B, 2016, 227, 117-127.
326 V. Singh, Z. Li, X. Zhou, X. Xu, J. Xu, A. Nand, H. Wen, H. Li, J. Zhu and J. Zhang, RSC Adv., 2016, 6, 3213-3218.

327 L. L. Yin, S. P. Wang, X. N. Shan, S. T. Zhang and N. J. Tao, Rev. Sci. Instrum., 2015, 86, 114101.

328 E. Kretschmann, Opt. Commun., 1972, 6, 185-187.

329 K. M. Shakesheff, X. Chen, M. C. Davies, A. Domb, C. J. Roberts, S. J. B. Tendler and P. M. Williams, Langmuir, 1995, 11, 3921-3927.

330 X. Chen, K. M. Shakesheff, M. C. Davies, J. Heller, C. J. Roberts, J. B. Tendler and P. M. Williams, J. Phys. Chem. B, 1995, 99, 11537-11542.

331 A. Baba, W. Knoll and R. Advincula, Rev. Sci. Instrum., 2006, 77, 064101.

332 L. Joergensen, B. Klösgen, A. C. Simonsen, J. Borch and E. Hagesaether, Int. J. Pharm., 2011, 411, 162-168.

333 K. Hall, T.-H. Lee, A. I. Mechler, M. J. Swann and M.-I. Aguilar, Sci. Rep., 2014, 4, 5479.

334 X. Chen, M. C. Davies, C. J. Roberts, K. M. Shakesheff, S. J. B. Tendler, P. M. Williams and J. Davies, J. Vac. Sci. Technol., B: Microelectron. Nanometer Struct.-Process., Meas., Phenom., 1996, 14, 1582-1586.

335 S. Kitamura and M. Iwatsuki, Appl. Phys. Lett., 1998, 72, 3154.

336 M. Nonnenmacher, M. P. Oboyle and H. K. Wickramasinghe, Appl. Phys. Lett., 1991, 58, 2921.

337 D. Kohl, P. Mesquida and G. Schitter, Microelectron. Eng., 2017, 176, 28-32.

338 H. M. I. Jaim, R. A. Isaacs, S. N. Rashkeev, M. Kuklja, D. P. Cole, M. C. LeMieux, I. Jasiuk, S. Nilufar and L. G. Salamanca-Riba, Carbon, 2016, 107, 56-66.

339 S. Gunther, B. Kaulich, L. Gregoratti and M. Kiskinova, Prog. Surf. Sci., 2002, 70, 187-260.

340 H. Luth, Solid Surfaces, Interfaces and Thin Films, Springer, Berlin, 2001.

341 J. C. Gonzalez, K. L. Bunker and P. E. Russell, Appl. Phys. Lett., 2001, 79, 1567.

342 T. Glatzel, S. Sadewasser and M. C. Lux-Steiner, Appl. Surf. Sci., 2003, 210, 84-89.

343 U. Zerweck, C. Loppacher, T. Otto, S. Grafström and L. M. Eng, Phys. Rev. B: Condens. Matter Mater. Phys., 2005, 71, 125424.

344 Z. M. Ma, L. Kou, Y. Naitoh, Y. J. Li and Y. Sugawara, Nanotechnology, 2013, 24, 225701.

345 L. Polak, S. d. Man and R. J. Wijngaarden, Rev. Sci. Instrum., 2014, 85, 046111.

346 S. Barbet, M. Popoff, H. Diesinger, D. Deresmes, D. Théron and T. Mélin, J. Appl. Phys., 2014, 115, 144313.

347 J. Murawski, T. Graupner, P. Milde, R. Raupach, U. Zerweck-Trogisch and L. M. Eng, J. Appl. Phys., 2015, 118, 154302.

348 L. Collins, A. Belianinov, S. Somnath, N. Balke, S. V. Kalinin and S. Jesse, Sci. Rep., 2016, 6, 30557.

349 R. Borgani, D. Forchheimer, J. Bergqvist, R. A. Thorén, O. Inganäs and D. B. Haviland, Appl. Phys. Lett., 2014, 105, 143113.

350 Y. Sugawara, L. Kou, Z. Ma, T. Kamijo, Y. Naitoh and L. Y. Jun, Appl. Phys. Lett., 2012, 100, 223104. 
351 M. Nonnenmacher, M. O'Boyle and H. K. Wickramasinghe, Ultramicroscopy, 1992, 44, 268-273.

352 A. K. Henning, T. Hochwitz, J. Slinkman, J. Never, S. Hoffmann, P. Kaszuba and C. Daghlian, J. Appl. Phys., 1995, 77, 1888.

353 N. Reitzel, T. Hassenkam, K. Balashev, T. R. Jensen, P. B. Howes, K. Kjaer, A. Fechtenkötter, N. Tchebotareva, S. Ito, K. Müllen and T. Bjørnholm, Chem.-Eur. J., 2001, 7, 4894-4901.

354 H. F. Knapp, P. Mesquida and A. Stemmer, Surf. Interface Anal., 2002, 33, 108-112.

355 M. Tosun, L. Chan, M. Amani, T. Roy, G. H. Ahn, P. Taheri, C. Carraro, J. W. Ager, R. Maboudian and A. Javey, ACS Nano, 2016, 10, 6853-6860.

356 U. Gysin, E. Meyer, T. Glatzel, G. Günzburger, H. R. Rossmann, T. A. Jung, S. Reshanov, A. Schöner and H. Bartolf, Microelectron. Eng., 2016, 160, 18-21.

357 C. Sommerhalter, T. Matthes, T. Glatzel, A. Jäger-Waldau and M. C. Lux-Steiner, Appl. Phys. Lett., 1999, 75, 286.

358 A. Findlay, D. Marinskiy, P. Edelman, M. Wilson, A. Savtchouk and J. Lagowski, ECS J. Solid State Sci. Technol., 2016, 5, P3087-P3095.

359 A. Yurtsever, D. Fernandez-Torre, J. Onoda, M. Abe, S. Morita, Y. Sugimoto and R. Perez, Nanoscale, 2017, 9, 5812-5821.

360 T. Spath, M. Popp, C. Perez Leon, M. Marz and R. Hoffmann-Vogel, Nanoscale, 2017, 9, 7868-7874.

361 Y. Yamagishi, K. Kobayashi, K. Noda and H. Yamada, Appl. Phys. Lett., 2016, 108, 093302.

362 G. Sauerbrey, Z. Phys. Chem., 1959, 155, 206.

363 F. Iwata, K. Saruta and A. Sasaki, Appl. Phys. A: Mater. Sci. Process., 1998, 66, S463-S466.

364 J. M. Friedt, K. H. Choi, F. Frederix and A. Campitelli, J. Electrochem. Soc., 2003, 150, H229-H234.

365 E. L. Smith, J. C. Barron, A. P. Abbott and K. S. Ryder, Anal. Chem., 2009, 81, 8466-8471.

366 V. S. Popov, E. N. Subcheva, A. V. Shelaev, R. G. Pavelko, V. G. Sevastyanov and N. T. Kuznetsov, Theor. Found. Chem. Eng., 2014, 48, 518-523.

367 P. Rivière, T. Nypelö, O. J. Rojas, A. Klug, N. Mundigler and R. Wimmer, J. Therm. Anal. Calorim., 2017, 127, 2059-2074.

368 M. Yavari, S. Maruf, Y. Ding and H. Lin, J. Membr. Sci., 2017, 525, 399-408.

369 C. C. Williams and H. K. Wickramasinghe, Appl. Phys. Lett., 1986, 49, 1587-1589.

370 L. Cui, W. Jeong, V. Fernández-Hurtado, J. Feist, F. J. García-Vidal, J. C. Cuevas, E. Meyhofer and P. Reddy, Nat. Commun., 2017, 8, 14479.

371 K. Kim, B. Song, V. Fernández-Hurtado, W. Lee, W. Jeong, L. Cui, D. Thompson, J. Feist, M. T. H. Reid, F. J. GarcíaVidal, J. C. Cuevas, E. Meyhofer and P. Reddy, Nature, 2015, 528, 387-391.

372 A. Majumdar, J. Lai, M. Chandrachood, O. Nakabeppu, Y. Wu and Z. Shi, Rev. Sci. Instrum., 1995, 66, 3584-3592.

373 K. Luo, Z. Shi, J. Lai and A. Majumdar, Appl. Phys. Lett., 1996, 68, 325-327.
374 G. Mills, H. Zhou, A. Midha, L. Donaldson and J. M. R. Weaver, Appl. Phys. Lett., 1998, 72, 2900-2902.

375 I. W. Rangelow, T. Gotszalk, P. Grabiec, K. Edinger and N. Abedinov, Microelectron. Eng., 2001, 57-58, 737-748.

376 Y. Zhang, P. S. Dobson and J. M. R. Weaver, Microelectron. Eng., 2011, 88, 2435-2438.

377 A. Gaitas and Y. B. Gianchandani, Ultramicroscopy, 2006, 106, 874-880.

378 L. Aigouy, E. Saïdi, L. Lalouat, J. Labéguerie-Egéa, M. Mortier, P. Löw and C. Bergaud, J. Appl. Phys., 2009, 106, 074301.

379 O. Nakabeppu, M. Chandrachood, Y. Wu, J. Lai and A. Majumdar, Appl. Phys. Lett., 1995, 66, 694-696.

380 S. Gomès, A. Assy and P.-O. Chapuis, Phys. Status Solidi A, 2015, 212, 477-494.

381 J. Bodzenta, J. Juszczyk, A. Kazmierczak-Balata, P. Firek, A. Fleming and M. Chirtoc, Int. J. Thermophys., 2016, 37, 17.

382 A. Pavlov, Appl. Phys. Lett., 2004, 85, 2095-2097.

383 S. Sadat, A. Tan, Y. J. Chua and P. Reddy, Nano Lett., 2010, 10, 2613-2617.

384 A. Kittel, W. Müller-Hirsch, J. Parisi, S.-A. Biehs, D. Reddig and M. Holthaus, Phys. Rev. Lett., 2005, 95, 224301.

385 S. Heisig, H. U. Danzebrink, A. Leyk, W. Mertin, S. Münster and E. Oesterschulze, Ultramicroscopy, 1998, 71, 99-105.

386 T. Leinhos, M. Stopka and E. Oesterschulze, Appl. Phys. A: Mater. Sci. Process., 1998, 66, S65-S69.

387 H. H. Roh, J. S. Lee, D. L. Kim, J. Park, K. Kim, O. Kwon, S. H. Park, Y. K. Choi and A. Majumdar, J. Vac. Sci. Technol., B: Microelectron. Nanometer Struct.-Process., Meas., Phenom., 2006, 24, 2398-2404.

388 H. H. Roh, J. S. Lee, D. L. Kim, J. Park, K. Kim, O. Kwon, S. H. Park, Y. K. Choi and A. Majumdar, J. Vac. Sci. Technol., B: Microelectron. Nanometer Struct.-Process., Meas., Phenom., 2006, 24, 2405-2411.

389 O. Nakabeppu and T. Suzuki, J. Therm. Anal. Calorim., 2002, 69, 727-737.

390 K. Kim, J. Chung, G. Hwang, O. Kwon and J. S. Lee, ACS Nano, 2011, 5, 8700-8709.

391 A. Bontempi, T. P. Nguyen, R. Salut, L. Thiery, D. Teyssieux and P. Vairac, Rev. Sci. Instrum., 2016, 87, 063702.

392 A. Majumdar and J. Varesi, J. Heat Transfer, 1998, 120, 297305.

393 A. Dazzi, R. Prazeres, F. Glotin and J. M. Ortega, Opt. Lett., 2005, 30, 2388-2390.

394 S. Lefèvre, J. B. Saulnier, C. Fuentes and S. Volz, Superlattices Microstruct., 2004, 35, 283-288.

395 Y. Ezzahri, L. D. Patiño Lopez, O. Chapuis, S. Dilhaire, S. Grauby, W. Claeys and S. Volz, Superlattices Microstruct., 2005, 38, 69-75.

396 P. S. Dobson, J. M. R. Weaver and G. Mills, IEEE Sensors, 2007, pp. 708-711, DOI: 10.1109/ICSENS.2007.4388498.

397 P. Tovee, M. Pumarol, D. Zeze, K. Kjoller and O. Kolosov, J. Appl. Phys., 2012, 112, 114317.

398 E. Puyoo, S. Grauby, J.-M. Rampnoux, E. Rouvière and S. Dilhaire, J. Appl. Phys., 2011, 109, 024302. 
399 M. Timofeeva, A. Bolshakov, P. D. Tovee, D. A. Zeze, V. G. Dubrovskii and O. V. Kolosov, Ultramicroscopy, 2016, 162, 42-51.

400 F. Menges, P. Mensch, H. Schmid, H. Riel, A. Stemmer and B. Gotsmann, Nat. Commun., 2016, 7, 10874.

401 L. D. P. Lopez, S. Grauby, S. Dilhaire, M. Amine Salhi, W. Claeys, S. Lefèvre and S. Volz, Microelectron. J., 2004, 35, 797-803.

402 G. Hwang and O. Kwon, Int. J. Therm. Sci., 2016, 108, 81-88. 403 S. Gomes, P. O. Chapuis, F. Nepveu, N. Trannoy, S. Volz, B. Charlot, G. Tessier, S. Dilhaire, B. Cretin and P. Vairac, IEEE Trans. Compon. Packag. Technol., 2007, 30, 424-431.

404 G. B. M. Fiege, F. J. Niedernostheide, H. J. Schulze, R. Barthelmeß and L. J. Balk, Microelectron. Reliab., 1999, 39, 1149-1152.

405 M. Tortello, S. Colonna, M. Bernal, J. Gomez, M. Pavese, C. Novara, F. Giorgis, M. Maggio, G. Guerra, G. Saracco, R. S. Gonnelli and A. Fina, Carbon, 2016, 109, 390-401.

406 L. Shi, J. Zhou, P. Kim, A. Bachtold, A. Majumdar and P. L. McEuen, J. Appl. Phys., 2009, 105, 104306.

407 Y.-J. Yu, M. Y. Han, S. Berciaud, A. B. Georgescu, T. F. Heinz, L. E. Brus, K. S. Kim and P. Kim, Appl. Phys. Lett., 2011, 99, 183105.
408 F. Menges, H. Riel, A. Stemmer and B. Gotsmann, Nano Lett., 2012, 12, 596-601.

409 S. S. Kharintsev, E. A. Chernykh, A. I. Fishman, S. K. Saikin, A. M. Alekseev and M. K. Salakhov, J. Phys. Chem. C, 2017, 121, 3007-3012.

410 S. Volz, X. Feng, C. Fuentes, P. Guérin and M. Jaouen, Int. J. Thermophys., 2002, 23, 1645-1657.

411 Y. Zhang, E. E. Castillo, R. J. Mehta, G. Ramanath and T. Borca-Tasciuc, Rev. Sci. Instrum., 2011, 82, 024902.

412 T. Borca-Tasciuc, Annu. Rev. Heat Transfer, 2013, 16, 211258.

413 P. J. Newby, B. Canut, J.-M. Bluet, S. Gomès, M. Isaiev, R. Burbelo, K. Termentzidis, P. Chantrenne, L. G. Fréchette and V. Lysenko, J. Appl. Phys., 2013, 114, 014903.

414 S. Gomès, L. David, V. Lysenko, A. Descamps, T. Nychyporuk and M. Raynaud, J. Phys. D: Appl. Phys., 2007, 40, 6677.

415 M. Luna, J. Colchero and A. M. Baró, J. Phys. Chem. B, 1999, 103, 9576-9581.

416 M. Chirtoc, J. Gibkes, R. Wernhardt, J. Pelzl and A. Wieck, Rev. Sci. Instrum., 2008, 79, 8. 